\title{
Hyperbolic Wavelet Analysis of Classical Isotropic and Anisotropic Besov-Sobolev Spaces
}

\author{
Martin Schäfer ${ }^{1} \cdot$ Tino Ullrich $^{1} \cdot$ Béatrice Vedel $^{2}$ \\ Received: 19 December 2019 / Revised: 20 March 2021 / Accepted: 30 March 2021 / \\ Published online: 18 May 2021 \\ (c) The Author(s) 2021
}

\begin{abstract}
In this paper we introduce new function spaces which we call anisotropic hyperbolic Besov and Triebel-Lizorkin spaces. Their definition is based on a hyperbolic Littlewood-Paley analysis involving an anisotropy vector only occurring in the smoothness weights. Such spaces provide a general and natural setting in order to understand what kind of anisotropic smoothness can be described using hyperbolic wavelets (in the literature also sometimes called tensor-product wavelets), a wavelet class which hitherto has been mainly used to characterize spaces of dominating mixed smoothness. A centerpiece of our present work are characterizations of these new spaces based on the hyperbolic wavelet transform. Hereby we treat both, the standard approach using wavelet systems equipped with sufficient smoothness, decay, and vanishing moments, but also the very simple and basic hyperbolic Haar system. The second major question we pursue is the relationship between the novel hyperbolic spaces and the classical anisotropic Besov-Lizorkin-Triebel scales. As our results show, in general, both approaches to resolve an anisotropy do not coincide. However, in the Sobolev range this is the case, providing a link to apply the newly obtained hyperbolic wavelet characterizations to the classical setting. In particular, this allows for detecting classical anisotropies via the coefficients of a universal hyperbolic wavelet basis, without the need of adaption of the basis or a-priori knowledge on the anisotropy.
\end{abstract}

Keywords Hyperbolic wavelet analysis · Hyperbolic Haar wavelet · Anisotropic Sobolev and Besov-Lizorkin-Triebel spaces

Dedicated to Prof. Hans Triebel on the occasion of his 85th birthday.

Communicated by Hans G. Feichtinger.

$凶 \quad$ Martin Schäfer

martin.schaefer@mathematik.tu-chemnitz.de

1 Chemnitz Technical University, 09111 Chemnitz, Germany

2 Université de Bretagne Sud, UMR 6205, LMBA, 56000 Vannes, France

Birkhäuser 
Mathematics Subject Classification 42C40 - 46E35 - 42B25 · 41A25

\section{Introduction}

With the development of wavelet analysis from the beginning of the 1980s until the present time we nowadays have several powerful tools at hand to perform signal analysis with the aim to extract important information out of a signal. The information is thereby usually coded in objects easy to compute and handle- the wavelet coefficients.

Wavelet methods have been used with the known success for the purpose of compression, denoising, classification, completion, etc., of data, to mention just a few. Roughly speaking, the common underlying idea is the fact that a few wavelet coefficients contain a rather complete information of the signal to be analyzed. However, due to their construction principle (dyadic dilations and integer translates of a few basic "mother" functions) classical wavelets are not well-suited for the analysis of, say, anisotropic signals. In fact, a signal which is rather smooth in $x$-direction but rough in $y$-direction (such as layers in the earth, stripes on a shirt, etc.) can not be properly resolved by a classical multi-resolution analysis. The respective wavelet coefficients do not contain the anisotropic smoothness information, they rather resolve a certain minimal smoothness. That results in a bad decay of the sequence of wavelet coefficients or, in other words, a bad compression rate.

Anisotropy is not a rare phenomenon since it arises whenever physics does not act the same in different directions, e.g., geophysics, oceanography, hydrology, fluid mechanics, or medical image processing (see $[4,41]$ among others) are some of the fields where it naturally appears. For this reason wavelets have been adapted in many different ways in order to "detect" and resolve anisotropy. There is a vast amount of literature dealing with this. For instance, there are wave atoms [14] as well as curvelets [7-9], shearlets [26,31,33], anisets, and anisotropic wavelets [28,50,51]. The latter concept represents a rather flexible construction since it can be build (theoretically) for any present anisotropy. The theoretical basis of anisotropic wavelet analysis is the equivalent characterization of corresponding anisotropic function spaces, like Hölder, Besov, Sobolev and Triebel-Lizorkin spaces. The major shortcoming of the existing theory is the fact that one has to know the anisotropy in advance, i.e., one has to adapt the wavelet accordingly. In other words, if physics does not provide the anisotropy parameters of the signal we are not able to resolve the signal accordingly without "trying out" several anisotropic bases. Such a method is, of course, hardly implementable in practice.

In Abry et. al. [1] it has been shown that any anisotropic Besov space-defined with respect to the cartesian axis - can "almost" be characterized with the help of the so-called hyperbolic wavelet transform. The anisotropy of the signal can then be detected using a uniform basis and is characterized by a special weight in the wavelet coefficients. This has led to an efficient algorithm for image classification and anisotropy detection applied to both synthetic and real textures (see [2,40]).

In this paper we further develop this idea of describing anisotropy with the help of the hyperbolic wavelet transform. For this reason we introduce a new family of anisotropic function spaces which are defined via a hyperbolic Littlewood-Paley analysis and 
for which we prove exact characterization with hyperbolic wavelets. The motivation behind this is to provide a general setting of anisotropic spaces characterized by one single basis of wavelets and thus to understand how one such fixed basis can help to describe anisotropic smoothness.

Concretely, we start with a hyperbolic Littlewood-Paley analysis defined as the usual tensor product

$$
\Delta_{\bar{j}} f:=\mathcal{F}^{-1}\left[\theta_{j_{1}} \otimes \ldots \otimes \theta_{j_{d}} \mathcal{F} f\right] \quad, \quad \bar{j}=\left(j_{1}, \ldots, j_{d}\right) \in \mathbb{N}_{0}^{d} .
$$

This hyperbolic decomposition of the frequency space has been widely used for the Fourier analytic definition of the well-known spaces with dominating mixed smoothness, see [42,59] and the references therein. These spaces represent a suitable framework for multivariate appoximation, see $[16,46]$ and the recent survey article [12]. The main ideas have been developed over more than fifty years of intense research in the former Soviet Union such that it is beyond the scope of this paper to name all the relevant references (cf. [12]).

Based on the decomposition (1), we then define spaces $\widetilde{A}_{p, q}^{s, \bar{\alpha}}\left(\mathbb{R}^{d}\right)$ with $A \in\{B, F\}$ of Besov-Lizorkin-Triebel type involving an anisotropy vector $\bar{\alpha}=\left(\alpha_{1}, \ldots, \alpha_{d}\right)>0$ with $\sum_{i=1}^{d} \alpha_{i}=d$. As a special case $(A=F, 1<p<\infty, q=2)$, these include the Sobolev type spaces $\widetilde{W}_{p}^{s, \bar{\alpha}}\left(\mathbb{R}^{d}\right):=\widetilde{F}_{p, 2}^{s, \bar{\alpha}}\left(\mathbb{R}^{d}\right)$, where

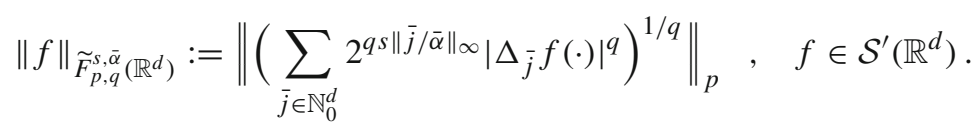

It is important to note that the anisotropy hereby only enters in the weight $2^{s\|\bar{j} / \bar{\alpha}\|_{\infty}}$, where we use the short-hand notation $\|\bar{j} / \bar{\alpha}\|_{\infty}:=\max \left\{\left|j_{1}\right| / \alpha_{1}, \ldots,\left|j_{d}\right| / \alpha_{d}\right\}$ for $\bar{j} \in$ $\mathbb{Z}^{d}$, but not in the choice of the Littlewood-Paley decomposition.

One of the main results of this paper is the coincidence (with respect to equivalent norms)

$$
\widetilde{W}_{p}^{s, \bar{\alpha}}\left(\mathbb{R}^{d}\right)=W_{p}^{s, \bar{\alpha}}\left(\mathbb{R}^{d}\right), \quad \text { if } 1<p<\infty,
$$

where the space on the right-hand side represents the classical anisotropic Sobolev space defined in (4) below. This relation has already been observed for isotropic (i.e. $\bar{\alpha}=(1, \ldots, 1))$ Hilbert-Sobolev spaces $(p=2)$ on the $d$-torus, see [13,25], as well as on $\mathbb{R}^{2}$ in [1]. Our result extends this observation to all $1<p<\infty$. Surprisingly, such a coincidence in the spirit of (2) is only possible in the Sobolev case. To be more precise, it holds

$$
\widetilde{A}_{p, q}^{s, \bar{\alpha}}\left(\mathbb{R}^{d}\right)=A_{p, q}^{s, \bar{\alpha}}\left(\mathbb{R}^{d}\right) \text { if and only if } A=F, 1<p<\infty \text {, and } q=2 .
$$

As an important consequence of this equality (2), we can further prove that it is possible to characterize (e.g. detect and classify) classical anisotropies described by the spaces $W_{p}^{s, \bar{\alpha}}\left(\mathbb{R}^{d}\right)$ via the wavelet coefficients of a universal hyperbolic wavelet basis. Compared to the classical approach using anisotropic wavelets, this approach 
has the advantage that one does not need a-priori knowledge on the anisotropies, otherwise required for constructing the "right" basis. In particular, our results entail that any sufficiently regular orthonormal basis $\left(\psi_{\bar{j}, \bar{k}}\right)_{\bar{j}, \bar{k}}$ of tensorized wavelets $\psi_{\bar{j}, \bar{k}}=$ $\psi_{j_{1}, k_{1}} \otimes \cdots \otimes \psi_{j_{d}, k_{d}}$ constitutes an unconditional Schauder basis for $W_{p}^{s, \bar{\alpha}}\left(\mathbb{R}^{d}\right)$, whose coefficients, measured in an appropriate corresponding sequence space, give rise to an equivalent norm on $W_{p}^{s, \bar{\alpha}}\left(\mathbb{R}^{d}\right)$, i.e. for $f \in W_{p}^{s, \bar{\alpha}}\left(\mathbb{R}^{d}\right)$ with coefficients $\left\langle f, \psi_{\bar{j}, \bar{k}}\right\rangle$

$$
\|f\|_{W_{p}^{s, \bar{\alpha}}\left(\mathbb{R}^{d}\right)} \asymp\left\|\left(\sum_{\bar{j} \in \mathbb{N}_{0}^{d}} 2^{2 s\|\bar{j} / \bar{\alpha}\|_{\infty}}\left|\sum_{\bar{k} \in \mathbb{Z}^{d}}\left\langle f, \psi_{\bar{j}, \bar{k}}\right\rangle \chi_{\bar{j}, \bar{k}}(\cdot)\right|^{2}\right)^{1 / 2}\right\|_{p} .
$$

This is stated in Theorem 6.3. A similar result, see Theorem 6.4, holds true for the hyperbolic Haar system $\mathcal{H}_{d}=\left(h_{\bar{j}, \bar{k}}\right)_{\bar{j}, \bar{k}}$, where $h_{\bar{j}, \bar{k}}=h_{j_{1}, k_{1}} \otimes \cdots \otimes h_{j_{d}, k_{d}}$, under the following restriction on the parameter $s$ of the space $W_{p}^{s, \bar{\alpha}}\left(\mathbb{R}^{d}\right)$,

$$
|s| / \alpha_{\min }<\min \left\{\frac{1}{p}, 1-\frac{1}{p}\right\} .
$$

In this direction, we would also like to mention the new and related findings of Oswald in [36] on the Schauder basis property of the hyperbolic Haar system in the classic isotropic Besov spaces defined via first-order moduli of smoothness.

At the center of our respective proofs, we will rely on discrete characterizations provided by hyperbolic wavelets for the spaces $\widetilde{A}_{p, q}^{s, \bar{\alpha}}\left(\mathbb{R}^{d}\right), A \in\{B, F\}$. These characterizations are fundamental and established in separate theorems, Theorem 4.6 (Remark 4.5) and Theorem 5.4 (Remark 5.5), whereby we follow two paths. On the one hand, we use the usual methodology and consider orthonormal wavelet bases for which we assume sufficient smoothness, decay, and vanishing moments. As a byproduct, we thereby significantly extend the wavelet characterizations in $[57,59]$ for Besov-Lizorkin-Triebel spaces with dominating mixed smoothness. On the other hand, we use a hyperbolic Haar system, which does not fulfill smoothness conditions as before but nevertheless allows for characterization in a certain restricted parameter range. Here we expect larger ranges for higher order spline wavelets (see e.g. Section 2.5 and Remark 2.14 in the book [53]) but leave this investigation for the future. The methodology developed here in context of the Haar wavelet will certainly be useful.

Let us remark that analysis with the Haar wavelet has a long tradition (see e.g. [3,23, $39,48]$ ), the Haar wavelet being the oldest and simplest orthonormal wavelet, conceived as early as 1910 [27]. Besides its elegance and simplicity, notably its connection to the Faber system [18] and other spline functions, such as e.g. the Chui-Wang wavelet [11], makes it interesting from a numerical perspective (see also [15]). Fast implementations are possible based on the fast Haar transform [29,38] and variations thereof (see e.g. [10]). In particular in imaging science it plays an important role in practical applications (here we refer to the survey [37]). Lastly, we would like to mention recent progress on basis properties of Haar systems in multivariate Besov-Lizorkin-Triebel spaces (see e.g. [22,36,43]). A short summary on this topic is given by Triebel in [54, Sect. 3.5] (see especially Remarks 3.20 and 3.21 there). 
The paper has the following structure. After having recalled in Sect. 2 some helpful Fourier analytic tools (in particular some classical maximal functions and associated inequalities) as well as the definition of the classical (anisotropic) function spaces $A_{p, q}^{s, \bar{\alpha}}\left(\mathbb{R}^{d}\right)$, where $A \in\{B, F\}$, we introduce in Sect. 3 the notion of hyperbolic Littlewood-Paley analysis and the related Besov-Lizorkin-Triebel spaces $\widetilde{A}_{p, q}^{s, \bar{\alpha}}\left(\mathbb{R}^{d}\right)$. Wavelet characterizations of these new hyperbolic spaces are the topic of Sects. 4 and 5, whereby we first resort to standard wavelets with sufficient smoothness, decay, and vanishing moments in Sect. 4, while in Sect. 5 we utilize a hyperbolic Haar basis. The relationship of the new scale to the traditional spaces is finally investigated in Sects. 6 and 7. Specifically, in Sect. 6, we show the equality $\widetilde{W}_{p}^{s, \bar{\alpha}}\left(\mathbb{R}^{d}\right)=W_{p}^{s, \bar{\alpha}}\left(\mathbb{R}^{d}\right)$, i.e. $\widetilde{F}_{p, 2}^{s, \bar{\alpha}}\left(\mathbb{R}^{d}\right)=F_{p, 2}^{s, \bar{\alpha}}\left(\mathbb{R}^{d}\right)$, in the range $1<p<\infty$, from which we can then extract our main theorems concerning hyperbolic wavelet characterizations of the classical $W_{p}^{s, \bar{\alpha}}\left(\mathbb{R}^{d}\right)$.

Let us agree on the following general notation. As usual $\mathbb{N}$ shall denote the natural numbers. We further put $\mathbb{N}_{0}:=\mathbb{N} \cup\{0\}$, and let $\mathbb{Z}$ denote the integers, $\mathbb{R}$ the real numbers, and $\mathbb{C}$ the complex numbers. By $\mathbb{T}:=\mathbb{R} / 2 \pi \mathbb{Z}$ we refer to the torus identified with the interval $[0,2 \pi] \subset \mathbb{R}$. We write $\langle x, y\rangle$ or $x \cdot y$ for the Euclidean inner product in $\mathbb{R}^{d}$ or $\mathbb{C}^{d}$. The letter $d$ is hereby always reserved for the underlying dimension and by $[d]$ we mean the set $\{1, \ldots, d\}$. For $0<p \leq \infty$ and $x \in \mathbb{R}^{d}$ we define $\|x\|_{p}:=\left(\sum_{i=1}^{d}\left|x_{i}\right|^{p}\right)^{1 / p}$, with the usual modification in the case $p=\infty$. If $1 \leq$ $p \leq \infty$ we set $p^{\prime}$ such that $1 / p+1 / p^{\prime}=1$. For $0<p, q \leq \infty$ we further denote $\sigma_{p, q}:=\max \{1 / p-1,1 / q-1,0\}$ and $\sigma_{p}:=\max \{1 / p-1,0\}$. We also put $x_{+}:=\left(\left(x_{1}\right)_{+}, \ldots,\left(x_{d}\right)_{+}\right)$, whereby $a_{+}:=\max \{a, 0\}$ for $a \in \mathbb{R}$. Analogously we define $x_{-}$. By $\left(x_{1}, \ldots, x_{d}\right)>0$ we shall mean that each coordinate is positive. Finally, as usual, $a \in \mathbb{R}$ is decomposed into $a=\lfloor a\rfloor+\{a\}$, where $0 \leq\{a\}<1$ and $\lfloor a\rfloor \in \mathbb{Z}$. In case $x \in \mathbb{R}^{d},\{x\}$ and $\lfloor x\rfloor$ are then meant component-wise. Multivariate indices are typesetted with a bar, like e.g. $\bar{k}, \bar{j}, \bar{\ell}$, or $\bar{m}$, to indicate the multi-index. In all the paper, the multi-index $\bar{\alpha}=\left(\alpha_{1}, \ldots, \alpha_{d}\right)>0$ thereby stands for an anisotropy and is such that $\alpha_{1}+\ldots+\alpha_{d}=d$. In addition, we here use the abbreviations $\alpha_{\min }:=\min \left\{\alpha_{1}, . ., \alpha_{d}\right\}$ and $\alpha_{\max }:=\max \left\{\alpha_{1}, . ., \alpha_{d}\right\}$. The notation $\bar{j} / \bar{\alpha}$ shall always stand for $\left(j_{1} / \alpha_{1}, \ldots, j_{d} / \alpha_{d}\right)$. Given a positive real $a>0$, we further write $a^{\bar{\alpha}}$ for the vector $\left(a^{\alpha_{1}}, \ldots, a^{\alpha_{d}}\right)$ and let $f\left(a^{\bar{\alpha}} x\right):=f\left(a^{\alpha_{1}} x_{1}, \ldots, a^{\alpha_{d}} x_{d}\right)$ be the anisotropically scaled version of the function $f: \mathbb{R}^{d} \rightarrow \mathbb{C}$. For two (quasi-)normed spaces $X$ and $Y$, the (quasi-)norm of an element $x \in X$ will be denoted by $\|x\|_{X}$. The symbol $X \hookrightarrow Y$ indicates that the identity operator is continuous. For two sequences $a_{n}$ and $b_{n}$ we will write $a_{n} \lesssim b_{n}$ if there exists a constant $c>0$ such that $a_{n} \leq c b_{n}$ for all $n$. We will write $a_{n} \asymp b_{n}$ if $a_{n} \lesssim b_{n}$ and $b_{n} \lesssim a_{n}$.

\section{Classical Spaces and Tools from Fourier Analysis}

Let $L_{p}=L_{p}\left(\mathbb{R}^{d}\right), 0<p \leq \infty$, be the Lebesgue space of all measurable functions $f: \mathbb{R}^{d} \rightarrow \mathbb{C}$ such that

$$
\|f\|_{p}:=\left(\int_{\mathbb{R}^{d}}|f(x)|^{p} d x\right)^{1 / p}<\infty
$$


with the usual modification if $p=\infty$. We will also need $L_{p}$-spaces on compact domains $\Omega \subset \mathbb{R}^{d}$ instead of $\mathbb{R}^{d}$ and shall write $\|f\|_{L_{p}(\Omega)}$ for the corresponding restricted $L_{p}$-(quasi-)norms.

For $k \in \mathbb{N}_{0}$, we denote by $C_{0}^{k}\left(\mathbb{R}^{d}\right)$ the collection of all compactly supported functions $\varphi$ on $\mathbb{R}^{d}$ which have uniformly continuous derivatives $D^{\bar{\gamma}} \varphi$ on $\mathbb{R}^{d}$ whenever $\|\bar{\gamma}\|_{1} \leq k$. Additionally, we define the spaces of infinitely differentiable functions $C^{\infty}\left(\mathbb{R}^{\bar{d}}\right)$ and infinitely differentiable functions with compact support $C_{0}^{\infty}\left(\mathbb{R}^{d}\right)$ as well as the Schwartz space $\mathcal{S}=\mathcal{S}\left(\mathbb{R}^{d}\right)$ of all rapidly decaying infinitely differentiable functions on $\mathbb{R}^{d}$, i.e.,

$$
\mathcal{S}\left(\mathbb{R}^{d}\right):=\left\{\varphi \in C^{\infty}\left(\mathbb{R}^{d}\right):\|\varphi\|_{k, \ell}<\infty \text { for all } k, \ell \in \mathbb{N}_{0}\right\}
$$

and

$$
\|\varphi\|_{k, \ell}:=\left\|(1+|\cdot|)^{k} \sum_{\|\bar{\gamma}\|_{1} \leq \ell}\left|D^{\bar{\gamma}} \varphi(\cdot)\right|\right\|_{\infty} \quad, \quad k, \ell \in \mathbb{N}_{0} .
$$

The space $\mathcal{S}^{\prime}\left(\mathbb{R}^{d}\right)$, the topological dual of $\mathcal{S}\left(\mathbb{R}^{d}\right)$, is also referred to as the space of tempered distributions on $\mathbb{R}^{d}$. Indeed, a linear mapping $f: \mathcal{S}\left(\mathbb{R}^{d}\right) \rightarrow \mathbb{C}$ belongs to $\mathcal{S}^{\prime}\left(\mathbb{R}^{d}\right)$ if and only if there exist numbers $k, \ell \in \mathbb{N}$ and a constant $c=c_{f}$ such that

$$
|f(\varphi)| \leq c_{f}\|\varphi\|_{k, \ell}
$$

for all $\varphi \in \mathcal{S}\left(\mathbb{R}^{d}\right)$. If $f \in \mathcal{S}^{\prime}\left(\mathbb{R}^{d}\right)$ is a regular distribution it can be identified with a locally integrable function on $\mathbb{R}^{d}$ in the sense that

$$
f(\varphi)=\int_{\mathbb{R}^{d}} f(x) \varphi(x) d x \quad, \quad \varphi \in \mathcal{S}\left(\mathbb{R}^{d}\right) .
$$

The space $\mathcal{S}^{\prime}\left(\mathbb{R}^{d}\right)$ is equipped with the weak*-topology.

For $f \in L_{1}\left(\mathbb{R}^{d}\right)$ we define the Fourier transform

$$
\mathcal{F} f(\xi)=(2 \pi)^{-d / 2} \int_{\mathbb{R}^{d}} f(y) e^{-i \xi \cdot y} d y, \quad \xi \in \mathbb{R}^{d},
$$

and the corresponding inverse Fourier transform $\mathcal{F}^{-1} f(\xi)=\mathcal{F} f(-\xi)$. As usual, the Fourier transform can be extended to $\mathcal{S}^{\prime}\left(\mathbb{R}^{d}\right)$ by $(\mathcal{F} f)(\varphi):=f(\mathcal{F} \varphi)$, where $f \in \mathcal{S}^{\prime}\left(\mathbb{R}^{d}\right)$ and $\varphi \in \mathcal{S}\left(\mathbb{R}^{d}\right)$. The mapping $\mathcal{F}: \mathcal{S}^{\prime}\left(\mathbb{R}^{d}\right) \rightarrow \mathcal{S}^{\prime}\left(\mathbb{R}^{d}\right)$ is a bijection.

The convolution $\varphi * \psi$ of two square-integrable functions $\varphi, \psi$ is defined via the integral

$$
(\varphi * \psi)(x)=\int_{\mathbb{R}^{d}} \varphi(x-y) \psi(y) d y .
$$

If $\varphi, \psi \in \mathcal{S}\left(\mathbb{R}^{d}\right)$ then $\varphi * \psi$ still belongs to $\mathcal{S}\left(\mathbb{R}^{d}\right)$. In fact, we have $\varphi * \psi \in C^{\infty}\left(\mathbb{R}^{d}\right)$ with at most polynomial growth even if $\varphi \in \mathcal{S}\left(\mathbb{R}^{d}\right)$ and $\psi \in L_{1}\left(\mathbb{R}^{d}\right)$. The convolution 
can be extended to $\mathcal{S}\left(\mathbb{R}^{d}\right) \times \mathcal{S}^{\prime}\left(\mathbb{R}^{d}\right)$ via $(\varphi * \psi)(x)=\psi(\varphi(x-\cdot))$, which makes sense pointwise and is a $C^{\infty}$-function on $\mathbb{R}^{d}$.

\subsection{Classical (an)isotropic Littlewood-Paley Analysis}

Subsequently, $\bar{\alpha}=\left(\alpha_{1}, \ldots, \alpha_{d}\right)>0$ will denote an anisotropy and be such that $\alpha_{1}+$ $\ldots+\alpha_{d}=d$. Anisotropic Besov spaces may then be introduced using an anisotropic Littlewood-Paley analysis depending on $\bar{\alpha}$. Classical isotropic spaces-as a particular case of anisotropic spaces - will thereby be obtained for $\bar{\alpha}=(1,1, \ldots, 1)$.

Let $\varphi_{0}^{\bar{\alpha}} \geq 0$ belong to the Schwartz class $\mathcal{S}\left(\mathbb{R}^{d}\right)$ and be such that, for $\xi=$ $\left(\xi_{1}, \ldots, \xi_{d}\right) \in \mathbb{R}^{d}$,

$$
\begin{aligned}
& \varphi_{0}^{\bar{\alpha}}(\xi)=1 \text { if } \sup _{i=1,2, \ldots, d}\left|\xi_{i}\right| \leq 1 \\
& \text { and } \varphi_{0}^{\bar{\alpha}}(\xi)=0 \text { if } \sup _{i=1, \ldots, d}\left|2^{-\alpha_{i}} \xi_{i}\right| \geq 1
\end{aligned}
$$

For $j \in \mathbb{N}$, further define

$$
\begin{aligned}
\varphi_{j}^{\bar{\alpha}}(\xi) & :=\varphi_{0}^{\bar{\alpha}}\left(2^{-j \bar{\alpha}} \xi\right)-\varphi_{0}^{\bar{\alpha}}\left(2^{-(j-1) \bar{\alpha}} \xi\right) \\
& =\varphi_{0}^{\bar{\alpha}}\left(2^{-j \alpha_{1}} \xi_{1}, \ldots, 2^{-j \alpha_{d}} \xi_{d}\right)-\varphi_{0}^{\bar{\alpha}}\left(2^{-(j-1) \alpha_{1}} \xi_{1}, \ldots, 2^{-(j-1) \alpha_{d}} \xi_{d}\right) .
\end{aligned}
$$

Then $\sum_{j=0}^{\infty} \varphi_{j}^{\bar{\alpha}} \equiv 1$, and $\left(\varphi_{j}^{\bar{\alpha}}\right)_{j \geq 0}$ is called an anisotropic resolution of unity. It satisfies

$$
\begin{aligned}
& \operatorname{supp}\left(\varphi_{0}^{\bar{\alpha}}\right) \subset R_{1}^{\bar{\alpha}}, \quad \operatorname{supp}\left(\varphi_{j}^{\bar{\alpha}}\right) \subset R_{j+1}^{\bar{\alpha}} \backslash R_{j-1}^{\bar{\alpha}}, \\
& \text { where } R_{j}^{\bar{\alpha}}=\left\{\xi=\left(\xi_{1}, \ldots, \xi_{d}\right) \in \mathbb{R}^{d}:\left|\xi_{i}\right| \leq 2^{\alpha_{i} j} \text { for } i \in[d]=\{1, \ldots, d\}\right\}
\end{aligned}
$$

For $f \in \mathcal{S}^{\prime}\left(\mathbb{R}^{d}\right)$, we then define

$$
\Delta_{j}^{\bar{\alpha}} f:=\mathcal{F}^{-1}\left(\varphi_{j}^{\bar{\alpha}} \mathcal{F} f\right)
$$

The sequence $\left(\Delta_{j}^{\bar{\alpha}} f\right)_{j \geq 0}$ is called an anisotropic Littlewood-Paley analysis of $f$. With this tool, the anisotropic Besov spaces are now defined as follows (see $[5,6])$.

Definition 2.1 For $0<p \leq \infty, 0<q \leq \infty, s \in \mathbb{R}$, the Besov space $B_{p, q}^{s, \bar{\alpha}}\left(\mathbb{R}^{d}\right)$ is defined by

$$
B_{p, q}^{s, \bar{\alpha}}\left(\mathbb{R}^{d}\right)=\left\{f \in \mathcal{S}^{\prime}\left(\mathbb{R}^{d}\right):\left(\sum_{j \geq 0} 2^{j s q}\left\|\Delta_{j}^{\bar{\alpha}} f\right\|_{p}^{q}\right)^{1 / q}<\infty\right\}
$$

with the usual modification for $q=\infty$. 
This definition does not depend on chosen resolution of unity $\varphi_{0}^{\bar{\alpha}}$ and the quantity

$$
\|f\|_{B_{p, q}^{s, \bar{\alpha}}}=\left(\sum_{j \geq 0} 2^{j s q}\left\|\Delta_{j}^{\bar{\alpha}} f\right\|_{p}^{q}\right)^{1 / q}
$$

is a norm (resp. quasi-norm) on $B_{p, q}^{s, \bar{\alpha}}\left(\mathbb{R}^{d}\right)$ for $1 \leq p, q \leq \infty(\operatorname{resp} .0<\min \{p, q\}<$ 1) and with the usual modification if $q=\infty$.

As in the isotropic case, anisotropic Besov spaces encompass a large class of classical anisotropic function spaces (see [51] for details). For example, when $p=q=2$, the Besov spaces coincide with the anisotropic Sobolev spaces and, when $p=q=\infty$, the spaces $B_{\infty, \infty}^{s, \bar{\alpha}}\left(\mathbb{R}^{d}\right)$ are called anisotropic Hölder spaces and are denoted by $\mathcal{C}^{s, \bar{\alpha}}\left(\mathbb{R}^{d}\right)$.

Definition 2.2 For $0<p<\infty, 0<q \leq \infty, s \in \mathbb{R}$, the Triebel-Lizorkin space $F_{p, q}^{s, \bar{\alpha}}\left(\mathbb{R}^{d}\right)$ is defined by

$$
F_{p, q}^{s, \bar{\alpha}}\left(\mathbb{R}^{d}\right)=\left\{f \in \mathcal{S}^{\prime}\left(\mathbb{R}^{d}\right):\left\|\left(\sum_{j \geq 0} 2^{j s q}\left|\Delta_{j}^{\bar{\alpha}} f(\cdot)\right|^{q}\right)^{1 / q}\right\|_{p}<\infty\right\},
$$

with the usual modification for $q=\infty$.

This definition does not depend on the chosen resolution of unity $\varphi_{0}^{\bar{\alpha}}$ and the quantity

$$
\|f\|_{F_{p, q}^{s, \bar{\alpha}}}=\left\|\left(\sum_{j \geq 0} 2^{j s q}\left|\Delta_{j}^{\bar{\alpha}} f(\cdot)\right|^{q}\right)^{1 / q}\right\|_{p}
$$

is a norm (resp. quasi-norm) on $F_{p, q}^{s, \bar{\alpha}}\left(\mathbb{R}^{d}\right)$ for $1 \leq p<\infty$ and $1 \leq q \leq \infty$ (resp. $0<\min \{p, q\}<1)$ and with the usual modification if $q=\infty$.

If $q=2$ and $1<p<\infty$, the anisotropic Triebel-Lizorkin space coincides with the anisotropic Sobolev space denoted by $W_{p}^{s, \bar{\alpha}}\left(\mathbb{R}^{d}\right)$ :

$$
W_{p}^{s, \bar{\alpha}}=\left\{f \in \mathcal{S}^{\prime}\left(\mathbb{R}^{d}\right):\left\|\mathcal{F}^{-1}\left[\left(\sum_{i=1}^{d}\left(1+\xi_{i}^{2}\right)^{1 / 2 \alpha_{i}}\right)^{s} \mathcal{F} f(\xi)\right]\right\|_{p}<\infty\right\}
$$

Remark 2.3 (i) As mentioned before, if $\bar{\alpha}=(1, \ldots, 1)$, it is easy to check that the spaces $B_{p, q}^{s, \bar{\alpha}}\left(\mathbb{R}^{d}\right)\left(\right.$ resp. $\left.F_{p, q}^{s, \bar{\alpha}}\left(\mathbb{R}^{d}\right)\right)$ coincide with the classical spaces $B_{p, q}^{s}\left(\mathbb{R}^{d}\right)$ (resp. $\left.F_{p, q}^{s}\left(\mathbb{R}^{d}\right)\right)$. In addition, we have $F_{p, 2}^{0, \bar{\alpha}}\left(\mathbb{R}^{d}\right)=L_{p}\left(\mathbb{R}^{d}\right)$ in the range $1<p<\infty$.

(ii) Our understanding of anisotropic spaces coincides with the one in Triebel [51] (see also the references therein). There are different (but related) notions of anisotropy in the Russian literature, see Nikol'skij [35, Chapt. 4] or Temlyakov [46, II.3]. A consequence of our Theorem 6.1 below is the fact that in case of $W$-spaces the mentioned approaches coincide and lead to the same notion of anisotropy. However, in case of Besov-Nikol'skij spaces this is in general not the case as for instance Theorem 7.1 shows. 


\subsection{Maximal Inequalities}

Let us provide here the maximal inequalities for the Hardy-Littlewood and Peetre maximal functions as required later. For further details we refer to [59, Chapt. 1] or [42, Chapt. 2].

We need a direction-wise version of the classical Hardy-Littlewood maximal operator acting on the $i$-th variable. For a locally integrable function $f: \mathbb{R}^{d} \rightarrow \mathbb{C}$ we let $M_{i} f$ denote the (centered) Hardy-Littlewood type maximal function given by

$$
\left(M_{i} f\right)(x)=\sup _{s>0} \frac{1}{2 s} \int_{x_{i}-s}^{x_{i}+s}\left|f\left(x_{1}, \ldots, x_{i-1}, t, x_{i+1}, \ldots, x_{d}\right)\right| d t \quad, \quad x \in \mathbb{R}^{d}
$$

We further denote the composition of these operators by $M_{e}=\prod_{i \in e} M_{i}$, where $e$ is a subset of $[d]=\{1, \ldots, d\}, M_{\ell} M_{k}$ has to be interpreted as $M_{\ell} \circ M_{k}$, and the product is taken in descending order of the elements of $e$.

The following variant of the Fefferman-Stein maximal inequality [19], i.e. the vector-valued generalization of the classical Hardy-Littlewood maximal inequality, is due to Stöckert [45] and valid in the direction-wise setting.

Theorem 2.4 ([45]) For $1<p<\infty$ and $1<q \leq \infty$ there exists a constant $c>0$ such that for any $i \in[d]$

$$
\left\|\left(\sum_{\ell \in \mathbb{N}}\left|M_{i} f_{\ell}\right|^{q}\right)^{1 / q}\right\|_{p} \leq c\left\|\left(\sum_{\ell \in \mathbb{N}}\left|f_{\ell}\right|^{q}\right)^{1 / q}\right\|_{p}
$$

holds for all sequences $\left\{f_{\ell}\right\}_{\ell \in \mathbb{N}}$ of locally Lebesgue-integrable functions on $\mathbb{R}^{d}$.

Remark 2.5 Iteration of this theorem yields a similar boundedness property for the operators $M_{e}$ with $e \subset[d]$. Furthermore, it should be noted that the uncentered versions of these operators also have similar properties due to the relation

$$
\left(M_{i} f\right)(x) \leq \sup _{s, t>0} \frac{1}{s+t} \int_{x_{i}-s}^{x_{i}+t}\left|f\left(x_{1}, \ldots, x_{i-1}, u, x_{i+1}, \ldots, x_{d}\right)\right| d u \leq 2\left(M_{i} f\right)(x) \text {. }
$$

The following construction of a maximal function is due to Peetre, Fefferman, and Stein. Let $\bar{b}=\left(b_{1}, \ldots, b_{d}\right)>0, a>0$, and $f \in L_{1}\left(\mathbb{R}^{d}\right)$ with $\mathcal{F} f$ compactly supported. We define the Peetre maximal function $P_{\bar{b}, a} f$ by

$$
P_{\bar{b}, a} f(x)=\sup _{z \in \mathbb{R}^{d}} \frac{|f(x-z)|}{\left(1+\left|b_{1} z_{1}\right|\right)^{a} \cdot \ldots \cdot\left(1+\left|b_{d} z_{d}\right|\right)^{a}} .
$$

Details for the following lemma can be found in [42, 1.6.4].

Lemma 2.6 ([42, 1.6.4]) Let $\Omega \subset \mathbb{R}^{d}$ be a compact set. Let further $a>0$ and $\bar{\gamma}=$ $\left(\gamma_{1}, \ldots, \gamma_{d}\right) \in \mathbb{N}_{0}^{d}$. Then there exist two constants $c_{1}, c_{2}>0$ (independent of $f$ ) such 
that

$$
\begin{aligned}
P_{(1, \ldots, 1), a}\left(D^{\bar{\gamma}} f\right)(x) & \leq c_{1} P_{(1, \ldots, 1), a} f(x) \\
& \leq c_{2}\left(M_{d}\left(M_{d-1}\left(\ldots\left(M_{1}|f|^{1 / a}\right) \ldots\right)\right)\right)^{a}(x)
\end{aligned}
$$

holds for all $f \in L_{1}\left(\mathbb{R}^{d}\right)$ with $\operatorname{supp}(\mathcal{F} f) \subset \Omega$ and all $x \in \mathbb{R}^{d}$. The constants $c_{1}, c_{2}$ depend on $\Omega$.

We finally give a vector-valued version of the Peetre maximal inequality which is a direct consequence of Lemma 2.6 together with Theorem 2.4.

Theorem 2.7 Let $0<p<\infty, 0<q \leq \infty$ and $a>\max \{1 / p, 1 / q\}$. Let further $\bar{b}^{\ell}=\left(b_{1}^{\ell}, \ldots, b_{d}^{\ell}\right)>0$ for $\ell \in \mathbb{N}$ and $\Omega=\left\{\Omega_{\ell}\right\}_{\ell \in \mathbb{N}}$, such that

$$
\Omega_{\ell} \subset\left[-b_{1}^{\ell}, b_{1}^{\ell}\right] \times \cdots \times\left[-b_{d}^{\ell}, b_{d}^{\ell}\right]
$$

is compact for $\ell \in \mathbb{N}$. Then there is a constant $C>0$ (independent of $f$ and $\Omega$ ) such that

$$
\left\|\left(\sum_{\ell \in \mathbb{N}}\left|P_{\bar{b}^{\ell}, a} f_{\ell}\right|^{q}\right)^{1 / q}\right\|_{p} \leq C\left\|\left(\sum_{\ell \in \mathbb{N}}\left|f_{\ell}\right|^{q}\right)^{1 / q}\right\|_{p}
$$

holds for all systems $f=\left\{f_{\ell}\right\}_{\ell \in \mathbb{N}}$ with $\operatorname{supp}\left(\mathcal{F} f_{\ell}\right) \subset \Omega_{\ell}, \ell \in \mathbb{N}$.

\section{Hyperbolic Littlewood-Paley Analysis}

Let $\theta_{0} \in \mathcal{S}(\mathbb{R})$ be a non-negative function supported on $[-2,2]$ with $\theta_{0}=1$ on $[-1,1]$. For any $j \in \mathbb{N}$, let us further define

$$
\theta_{j}=\theta_{0}\left(2^{-j} \cdot\right)-\theta_{0}\left(2^{-(j-1)} \cdot\right)
$$

such that $\left(\theta_{j}\right)_{j}$ is a univariate resolution of unity, i.e., $\sum_{j \geq 0} \theta_{j}(\cdot)=1$. Observe that, for any $j \geq 1, \operatorname{supp}\left(\theta_{j}\right) \subset\left\{2^{j-1} \leq|\xi| \leq 2^{j+1}\right\}$ and $\theta_{j}=\theta_{1}\left(2^{-(j-1)} \cdot\right)$.

Remark 3.1 In the following, the function $\theta_{0}$ can be chosen with an arbitrary compact support. It does not change the main results even if technical details of proofs and lemmas have to be adapted. This allows to choose $\theta_{0}$ as the Fourier transform of a Meyer scaling function.

Let us now come to the main concept of this paper, the hyperbolic Littlewood-Paley analysis.

Definition 3.2 (i) For any $\bar{j}=\left(j_{1}, \ldots, j_{d}\right) \in \mathbb{N}_{0}^{d}$ and any $\xi=\left(\xi_{1}, \ldots, \xi_{d}\right) \in \mathbb{R}^{d}$ set

$$
\theta_{\bar{j}}\left(\xi_{1}, \ldots, \xi_{d}\right):=\theta_{j_{1}}\left(\xi_{1}\right) \theta_{j_{2}}\left(\xi_{2}\right) \ldots \theta_{j_{d}}\left(\xi_{d}\right)
$$


The function $\theta_{\bar{j}}$ belongs to $\mathcal{S}\left(\mathbb{R}^{d}\right)$ for all $\bar{j} \in \mathbb{N}_{0}^{d}$ and is compactly supported on a dyadic rectangle. Further $\sum_{\bar{j} \in \mathbb{N}_{0}^{d}} \theta_{\bar{j}} \equiv 1$ and $\left(\theta_{\bar{j}}\right)_{j}$ is called a hyperbolic resolution of unity.

(ii) For $f \in \mathcal{S}^{\prime}\left(\mathbb{R}^{d}\right)$ and $\bar{j} \in \mathbb{N}_{0}^{d}$ set

$$
\Delta_{\bar{j}} f:=\mathcal{F}^{-1}\left(\theta_{\bar{j}} \mathcal{F} f\right) .
$$

The sequence $\left(\Delta_{\bar{j}} f\right)_{\bar{j} \in \mathbb{N}_{0}^{d}}$ is called a hyperbolic Littlewood-Paley analysis of $f$.

We are now in the position to introduce new functional spaces called anisotropic hyperbolic Besov spaces and anisotropic hyperbolic Triebel-Lizorkin spaces defined via the hyperbolic Littlewood-Paley analysis.

Definition 3.3 For $0<p \leq \infty, 0<q \leq \infty, s \in \mathbb{R}$, and $\bar{\alpha}=\left(\alpha_{1}, \ldots, \alpha_{d}\right)>0$ such that $\sum_{i=1}^{d} \alpha_{i}=d$ we define the anisotropic hyperbolic Besov space $\widetilde{B}_{p, q}^{s, \bar{\alpha}}\left(\mathbb{R}^{d}\right)$ via

$$
\widetilde{B}_{p, q}^{s, \bar{\alpha}}\left(\mathbb{R}^{d}\right)=\left\{f \in \mathcal{S}^{\prime}\left(\mathbb{R}^{d}\right):\left(\sum_{\bar{j} \in \mathbb{N}_{0}^{d}} 2^{\left\|\left(j_{1} / \alpha_{1}, \ldots, j_{d} / \alpha_{d}\right)\right\|_{\infty} s q}\left\|\Delta_{\bar{j}} f\right\|_{p}^{q}\right)^{1 / q}<\infty\right\}
$$

with the usual modification in case $q=\infty$. The quantity (with usual modification if $q=\infty)$

$$
\|f\|_{\widetilde{B}_{p, q}^{s, \bar{\alpha}}}:=\left(\sum_{\bar{j} \in \mathbb{N}_{0}^{d}} 2^{\left\|\left(j_{1} / \alpha_{1}, \ldots, j_{d} / \alpha_{d}\right)\right\|_{\infty} s q}\left\|\Delta_{\bar{j}} f\right\|_{p}^{q}\right)^{1 / q}
$$

is a norm (resp. quasi-norm) on $\widetilde{B}_{p, q}^{s, \bar{\alpha}}\left(\mathbb{R}^{d}\right)$ for $1 \leq p, q \leq \infty(\operatorname{resp.} 0<\min \{p, q\}<$ $1)$.

Definition 3.4 For $0<p<\infty, 0<q \leq \infty, s \in \mathbb{R}, \bar{\alpha}=\left(\alpha_{1}, \ldots, \alpha_{d}\right)>0$ such that $\sum_{i} \alpha_{i}=d$ we define the anisotropic hyperbolic Triebel-Lizorkin space via

$$
\widetilde{F}_{p, q}^{s, \bar{\alpha}}\left(\mathbb{R}^{d}\right)=\left\{f \in \mathcal{S}^{\prime}\left(\mathbb{R}^{d}\right):\left\|\left(\sum_{\bar{j} \in \mathbb{N}_{0}^{d}} 2^{\left\|\left(j_{1} / \alpha_{1}, \ldots, j_{d} / \alpha_{d}\right)\right\|_{\infty} s q}\left|\Delta_{\bar{j}} f(\cdot)\right|^{q}\right)^{1 / q}\right\|_{p}<\infty\right\},
$$

with the usual modification in case $q=\infty$. The quantity (with usual modification if $q=\infty)$

$$
\|f\|_{\widetilde{F}_{p, q}^{s, \bar{\alpha}}}:=\left\|\left(\sum_{\bar{j} \in \mathbb{N}_{0}^{d}} 2^{\left\|\left(j_{1} / \alpha_{1}, \ldots, j_{d} / \alpha_{d}\right)\right\|_{\infty} s q}\left|\Delta_{\bar{j}} f(\cdot)\right|^{q}\right)^{1 / q}\right\|_{p}
$$

is a norm on $\widetilde{F}_{p, q}^{s, \bar{\alpha}}\left(\mathbb{R}^{d}\right)$ for $1 \leq p<\infty, 1 \leq q \leq \infty$ (resp. quasi-norm for $0<$ $\min \{p, q\}<1$ ). 
Remark 3.5 The definitions of anisotropic hyperbolic Besov and Triebel-Lizorkin spaces given above do not depend on the chosen resolution of unity $\left(\theta_{\bar{j}}\right)_{\bar{j}}$. They include four indices: $s$ stands for the regularity, $p$ is the integration parameter and $q$ the socalled fine-index. The parameter $\bar{\alpha}=\left(\alpha_{1}, \ldots, \alpha_{d}\right)$ encodes the present anisotropy: the more $\alpha_{\min }=\min \left\{\alpha_{1}, \ldots, \alpha_{d}\right\}$ is close to 0 and $\alpha_{\max }=\max \left\{\alpha_{1}, \ldots, \alpha_{d}\right\}$ is close to $d$, the more we need directional smoothness in one axis compared to others. On the other hand, if $\bar{\alpha}=(1, \ldots, 1)$ the anisotropy becomes an "isotropy".

Remark 3.6 By analogy with the classical spaces, if $q=2$ and $1<p<\infty, \widetilde{F}_{p, q}^{s, \bar{\alpha}}\left(\mathbb{R}^{d}\right)$ is called anisotropic hyperbolic Sobolev space and is denoted by $\widetilde{W}_{p}^{s, \bar{\alpha}}\left(\mathbb{R}^{d}\right)$. In case $\bar{\alpha}=(1, \ldots, 1)$ we write $\widetilde{W}_{p}^{s}\left(\mathbb{R}^{d}\right)$.

Let us finally introduce classical spaces with dominating mixed smoothness in the spirit of [42,59].

Definition 3.7 Let $r \in \mathbb{R}, 0<p, q \leq \infty(p<\infty$ in the $F$-case).

(i) The Besov space with dominating mixed smoothness $S_{p, q}^{r} B\left(\mathbb{R}^{d}\right)$ is the collection of all distributions $f \in \mathcal{S}^{\prime}\left(\mathbb{R}^{d}\right)$ such that the following (quasi-)norm

$$
\|f\|_{S_{p, q}^{r} B\left(\mathbb{R}^{d}\right)}:=\left(\sum_{\bar{j} \in \mathbb{N}_{0}^{d}} 2^{r\|\bar{j}\|_{1} q}\left\|\Delta_{\bar{j}} f\right\|_{p}^{q}\right)^{1 / q} \text { is finite. }
$$

(ii) The Triebel-Lizorkin space with dominating mixed smoothness $S_{p, q}^{r} F\left(\mathbb{R}^{d}\right)$ is the collection of all distributions $f \in \mathcal{S}^{\prime}\left(\mathbb{R}^{d}\right)$ such that the following (quasi-)norm

$$
\|f\|_{S_{p, q}^{r} F\left(\mathbb{R}^{d}\right)}:=\left\|\left(\sum_{\bar{j} \in \mathbb{N}_{0}^{d}} 2^{r\|\bar{j}\|_{1} q}\left|\Delta_{\bar{j}} f(x)\right|^{q}\right)^{1 / q}\right\|_{p} \text { is finite. }
$$

(iii) If $1<p<\infty$ and $r \in \mathbb{R}$ then the Sobolev space with dominating mixed smoothness $S_{p}^{r} W\left(\mathbb{R}^{d}\right)$ is the collection of all $f \in \mathcal{S}^{\prime}\left(\mathbb{R}^{d}\right)$ such that

$$
\|f\|_{S_{p}^{r} W}:=\left\|\mathcal{F}^{-1}\left[\prod_{i=1}^{d}\left(1+\left|\xi_{i}\right|^{2}\right)^{r / 2} \mathcal{F} f\right]\right\|_{p} \text { is finite. }
$$

Let us also state a useful Fourier multiplier theorem, see [59, Thm. 1.12] or [42, p. 77].

Lemma 3.8 ([42,59]) Let $0<p<\infty, 0<q \leq \infty$, and $r>\frac{1}{\min (p, q)}+\frac{1}{2}$. Further, let $\left\{\Omega_{\bar{k}}\right\}_{\bar{k} \in \mathbb{N}_{0}^{d}}$ be a sequence of compact subsets of $\mathbb{R}^{d}$ such that

$$
\Omega_{\bar{k}} \subset\left\{x \in \mathbb{R}^{d}:\left|x_{i}\right| \leq 2^{k_{i}}, i=1, \ldots, d\right\} .
$$


Then, there is a positive constant $C>0$ such that

$$
\begin{aligned}
\left\|\left(\sum_{\bar{k} \in \mathbb{N}_{0}^{d}}\left|\mathcal{F}^{-1}\left[\rho_{\bar{k}} \mathcal{F} f_{\bar{k}}\right](\cdot)\right|^{q}\right)^{\frac{1}{q}}\right\|_{p} \leq & C\left\|\left(\sum_{\bar{k} \in \mathbb{N}_{0}^{d}}\left|f_{\bar{k}}(\cdot)\right|^{q}\right)^{\frac{1}{q}}\right\|_{p} \\
& \times \sup _{\bar{\ell} \in \mathbb{N}_{0}^{d}}\left\|\rho_{\bar{\ell}}\left(2^{\ell_{1}} \cdot \ldots, 2^{\ell_{d}} \cdot\right)\right\|_{S_{2}^{r} W}
\end{aligned}
$$

holds for all systems $\left\{f_{\bar{k}}\right\}_{\bar{k} \in \mathbb{N}_{0}^{d}} \in L_{p}\left(\ell_{q}\right)$ with $\operatorname{supp}\left(\mathcal{F} f_{\bar{k}}\right) \subset \Omega_{\bar{k}}$ and all systems $\left\{\rho_{\bar{k}}\right\}_{\bar{k} \in \mathbb{N}_{0}^{d}} \subset S_{2}^{r} W\left(\mathbb{R}^{d}\right)$.

\section{Hyperbolic Wavelet Analysis}

In this section we prove hyperbolic wavelet characterizations of the spaces $\widetilde{B}_{p, q}^{s, \bar{\alpha}}\left(\mathbb{R}^{d}\right)$ and $\widetilde{F}_{p, q}^{s, \bar{\alpha}}\left(\mathbb{R}^{d}\right)$ defined in Definitions 3.3 and 3.4, respectively. It should be noted that the proof technique used for Theorem 4.6 below also represents a progress towards new optimal wavelet characterizations of Besov-Lizorkin-Triebel spaces with dominating mixed smoothness, which extends the results in [59, Sect. 2.4] significantly, see Remark 4.8 below.

Let us start with univariate orthogonal wavelets given by a scaling function $\psi_{0}$ and a corresponding wavelet $\psi$. These functions are supposed to satisfy $\left\|\psi_{0}\right\|_{2}=\|\psi\|_{2}=1$ and the following (minimal) conditions:

(K) It holds $\psi_{0}, \psi \in C^{K}(\mathbb{R})$. For any $M \in \mathbb{N}$ there is a constant $C_{M}>0$ such that for all $0 \leq \alpha \leq K$ it holds

$$
\left|D^{\alpha} \psi_{0}(x)\right|+\left|D^{\alpha} \psi(x)\right| \leq C_{M}(1+|x|)^{-M} \quad, \quad x \in \mathbb{R} .
$$

(L) The wavelet $\psi$ has vanishing moments up to order $L-1$ : For $L>\beta \in \mathbb{N}_{0}$ it holds

$$
\int_{\mathbb{R}} \psi(x) x^{\beta} d x=0 .
$$

In case $L=0$ the condition is void.

We shall denote

$$
\psi_{j, k}:=\frac{1}{\sqrt{2}} \psi\left(2^{j-1} \cdot-k\right) \quad, j \in \mathbb{N}, k \in \mathbb{Z},
$$

and $\psi_{0, k}:=\psi_{0}(\cdot-k)$. In addition, we set $\psi_{j, k} \equiv 0$ if $j<0$, so that each $f \in L_{2}(\mathbb{R})$ has the expansion $\sum_{j, k \in \mathbb{Z}} 2^{|j|}\left\langle f, \psi_{j, k}\right\rangle_{L_{2}} \psi_{j, k}$. To obtain the hyperbolic wavelet basis in $L_{2}\left(\mathbb{R}^{d}\right)$ we tensorize over all scales and obtain

$$
\psi_{\bar{j}, \bar{k}}\left(x_{1}, \ldots, x_{d}\right):=\psi_{j_{1}, k_{1}}\left(x_{1}\right) \cdot \ldots \cdot \psi_{j_{d}, k_{d}}\left(x_{d}\right)
$$




$$
x=\left(x_{1}, \ldots, x_{d}\right) \in \mathbb{R}^{d}, \bar{j} \in \mathbb{Z}^{d}, \bar{k} \in \mathbb{Z}^{d}
$$

The following lemma recalls a useful convolution relation. Let us clarify the notation first. For a given univariate function $\Lambda$ we will use the notation $\Lambda_{j}(\cdot):=$ $2^{j-1} \Lambda\left(2^{j-1} \cdot\right), j \in \mathbb{N} . \Lambda_{0}$ is typically defined separately, and $\Lambda_{j} \equiv 0$ if $j<0$. For $j \in \mathbb{Z}$, we will further put $x_{j, m}:=2^{-j} m$ and $I_{j, m}:=\left[2^{-j} m, 2^{-j}(m+1)\right)$ with associated characteristic function $\chi_{j, m}:=\mathbb{1}_{I_{j, m}}$.

Lemma 4.1 Let $\Lambda_{0}, \Lambda \in \mathcal{S}(\mathbb{R})$ with $\Lambda$ having infinitely many vanishing moments, i.e.,

$$
\int_{\mathbb{R}} \Lambda(x) x^{\beta} d x=0
$$

for all $\beta \in \mathbb{N}$. Let further $\psi_{0}$ and $\psi$ satisfy $(K)$ and $(L)$ as above and $R>0$ be a given real number. Then it exists a constant $C_{R}>0$ such that for any $j \in \mathbb{N}_{0}$ and $\ell, m \in \mathbb{Z}$ the convolution relation

$$
\left|\left(\Lambda_{j} * \psi_{j+\ell, m}\right)(x)\right| \leq C_{R} 2^{-N_{\operatorname{sign}(\ell)}|\ell|}\left(1+2^{\min \{j, j+\ell\}}\left|x-x_{j+\ell, m}\right|\right)^{-R}
$$

holds true with $\operatorname{sign}(\ell) \in\{+,-, 0\}$ and $N_{0}:=0, N_{+}:=L+1$ and $N_{-}:=K$.

Proof The above lemma is a special case of a more general convolution relation, see for instance [24, p. 466] for the most general version. Originally, this relation is due to Frazier, Jawerth [20, Lem. 3.3], [21, Lem. B.1, B.2].

Lemma 4.1 immediately implies the following multivariate version by exploiting the tensor product structure. Similar as for the hyperbolic wavelet system, we use the notation

$$
\Lambda_{\bar{j}}(x):=\Lambda_{j_{1}}\left(x_{1}\right) \cdot \ldots \cdot \Lambda_{j_{d}}\left(x_{d}\right) \quad, \quad x \in \mathbb{R}^{d}, \bar{j} \in \mathbb{Z}^{d}
$$

In the sequel we will further need the notation

$$
Q_{\bar{j}, \bar{m}}:=I_{j_{1}, m_{1}} \times \ldots \times I_{j_{d}, m_{d}} \text { and } \chi_{\bar{j}, \bar{m}}\left(x_{1}, \ldots, x_{d}\right):=\chi_{j_{1}, m_{1}}\left(x_{1}\right) \cdot \ldots \cdot \chi_{j_{d}, m_{d}}\left(x_{d}\right)
$$

with the notation $I_{j_{i}, m_{i}}$ and $\chi_{j_{i}, m_{i}}, i \in[d]=\{1, \ldots, d\}$, introduced right before Lemma 4.1.

Lemma 4.2 Let $\Lambda, \Lambda_{0}, \psi_{0}, \psi$ as in Lemma 4.1. For any $R>0$ there exists a contant $C_{R}>0$ such that for any $\bar{j} \in \mathbb{N}_{0}^{d}$ and $\bar{\ell}, \bar{m} \in \mathbb{Z}^{d}$ the convolution relation

$$
\left|\left(\Lambda_{\bar{j}} * \psi_{\bar{j}+\bar{\ell}, \bar{m}}\right)(x)\right| \leq C_{R} \prod_{i=1}^{d} 2^{-N_{\operatorname{sign}\left(\ell_{i}\right)}\left|\ell_{i}\right|}\left(1+2^{\min \left\{j_{i}, j_{i}+\ell_{i}\right\}}\left|x_{i}-2^{-\left(j_{i}+\ell_{i}\right)} m_{i}\right|\right)^{-R}
$$

holds true with $\operatorname{sign}\left(\ell_{i}\right) \in\{+,-, 0\}$ and $N_{0}:=0, N_{+}:=L+1$ and $N_{-}:=K$. 
The next proposition is also crucial and represents a "hyperbolic version" of [30, Lem. 3.7]. An isotropic version is originally due to Kyriazis [32, Lem. 7.1]. For the convenience of the reader we give a proof.

Proposition 4.3 Let $0<r \leq 1$ and $R>1 / r$. For any sequence $\left(\lambda_{\bar{m}}\right)_{\bar{m} \in \mathbb{Z}^{d}}$ of complex numbers and any $\bar{j}, \bar{\ell} \in \mathbb{Z}^{d}$ we have, using the notation $\bar{\ell}_{+}=\left(\left(\ell_{1}\right)_{+}, \ldots,\left(\ell_{d}\right)_{+}\right)$,

$$
\begin{aligned}
& \sum_{\bar{m} \in \mathbb{Z}^{d}}\left|\lambda_{\bar{m}}\right| \prod_{i=1}^{d}\left(1+2^{\min \left\{j_{i}, j_{i}+\ell_{i}\right\}}\left|x_{i}-2^{-\left(j_{i}+\ell_{i}\right)} m_{i}\right|\right)^{-R} \\
& \lesssim 2^{\left\|\bar{\ell}_{+}\right\|_{1} / r}\left[M_{[d]} \mid \sum_{\bar{m} \in \mathbb{Z}^{d}} \lambda_{\bar{m}} \chi_{\bar{j}+\bar{\ell},\left.\bar{m}\right|^{r}}\right]^{1 / r}(x) \quad, x \in \mathbb{R}^{d}
\end{aligned}
$$

where $M_{[d]}=\prod_{i=1}^{d} M_{i}$ stands for the iterated Hardy-Littlewood maximal operator (see (5)).

Proof For $d=1$ the statement is equivalent to the original result from Kyriazis [32, Lem. 7.1] in one dimension. Subsequently, the case $d>1$ is proved by induction on $d$. Thereby, given $x=\left(x_{1}, \ldots, x_{d}\right) \in \mathbb{R}^{d}$, we use the notation $x_{[d-1]}$ for the vector $\left(x_{1}, \ldots, x_{d-1}\right) \in \mathbb{R}^{d-1}$. In the same manner, we define $\bar{j}_{[d-1]}, \bar{\ell}_{[d-1]}$, and $\bar{m}_{[d-1]}$. Using the statement for $d=1$ yields

$$
\begin{aligned}
& \sum_{\bar{m} \in \mathbb{Z}^{d}}\left|\lambda_{\bar{m}}\right| \prod_{i=1}^{d}\left(1+2^{\min \left\{j_{i}, j_{i}+\ell_{i}\right\}}\left|x_{i}-2^{-\left(j_{i}+\ell_{i}\right)} m_{i}\right|\right)^{-R} \\
& =\sum_{m_{d} \in \mathbb{Z}} \tilde{\lambda}_{m_{d}}\left[x_{[d-1]}\right]\left(1+2^{\min \left\{j_{d}, j_{d}+\ell_{d}\right\}}\left|x_{d}-2^{-\left(j_{d}+\ell_{d}\right)} m_{d}\right|\right)^{-R} \\
& \lesssim 2^{\frac{\left|\left(\ell_{d}\right)+\right|}{r}}\left[M_{d}\left|\sum_{m_{d} \in \mathbb{Z}} \tilde{\lambda}_{m_{d}}\left[x_{[d-1]}\right] \chi_{j_{d}+\ell_{d}, m_{d}}\right|^{r}\right]^{\frac{1}{r}}\left(x_{d}\right),
\end{aligned}
$$

where for $m_{d} \in \mathbb{Z}$

$$
\tilde{\lambda}_{m_{d}}\left[x_{[d-1]}\right]:=\sum_{\bar{m}_{[d-1]} \in \mathbb{Z}^{d-1}}\left|\lambda_{\bar{m}}\right| \prod_{i=1}^{d-1}\left(1+2^{\min \left\{j_{i}, j_{i}+\ell_{i}\right\}}\left|x_{i}-2^{-\left(j_{i}+\ell_{i}\right)} m_{i}\right|\right)^{-R} .
$$

Based on the induction hypothesis, we further have

$$
\begin{aligned}
& \sum_{m_{d} \in \mathbb{Z}} \tilde{\lambda}_{m_{d}}\left[x_{[d-1]}\right] \chi_{j_{d}+\ell_{d}, m_{d}}(\cdot) \\
& =\sum_{\bar{m}_{[d-1]} \in \mathbb{Z}^{d-1}}\left(\sum_{m_{d} \in \mathbb{Z}}\left|\lambda_{\bar{m}}\right| \chi_{j_{d}+\ell_{d}, m_{d}}(\cdot)\right) \prod_{i=1}^{d-1}\left(1+2^{\min \left\{j_{i}, j_{i}+\ell_{i}\right\}}\left|x_{i}-2^{-\left(j_{i}+\ell_{i}\right)} m_{i}\right|\right)^{-R}
\end{aligned}
$$




$$
\begin{aligned}
& \lesssim 2^{\frac{\left\|\left(\bar{\ell}_{[d-1]}\right)+\right\|_{1}}{r}}\left[M_{[d-1]} \mid \sum_{\bar{m}_{[d-1]} \in \mathbb{Z}^{d-1}}\left(\sum_{m_{d} \in \mathbb{Z}}\left|\lambda_{\bar{m}}\right| \chi_{j_{d}+\ell_{d}, m_{d}}(\cdot)\right) \chi_{\left.(\bar{j}+\bar{\ell})_{[d-1]},\left.\bar{m}_{[d-1]}\right|^{r}\right]^{\frac{1}{r}}\left(x_{[d-1]}\right)}\right. \\
& =2^{\frac{\|(\bar{\ell}[d-1])+\|_{1}}{r}}\left[M_{[d-1]} \mid \sum_{\bar{m} \in \mathbb{Z}^{d}} \lambda_{\bar{m}} \chi_{\left.\bar{j}+\bar{\ell},\left.\bar{m}\right|^{r}\right]^{\frac{1}{r}}\left(x_{[d-1]}, \cdot\right) .}\right.
\end{aligned}
$$

In view of the monotonicity of the operator $M_{d}$, inserting this inequality into the last line of (10) directly leads to the desired assertion.

Before stating our main result we need a further definition.

Definition 4.4 Let $0<q \leq \infty, s \in \mathbb{R}$, and $\bar{\alpha}=\left(\alpha_{1}, \ldots, \alpha_{d}\right)>0$ such that $\sum_{i=1}^{d} \alpha_{i}=$ $d$.

(i) If $0<p<\infty$ we define the sequence space $\tilde{f}_{p, q}^{s, \bar{\alpha}}$ as the collection of all sequences $(\lambda \bar{j}, \bar{k})_{\bar{j} \in \mathbb{N}_{0}^{d}, \bar{k} \in \mathbb{Z}^{d}} \subset \mathbb{C}$ such that the (quasi-)norm (usual modification in case $q=\infty$ )

$$
\begin{aligned}
& \left\|\left(\lambda_{\bar{j}, \bar{k}}\right)_{\bar{j} \in \mathbb{N}_{0}^{d}, \bar{k} \in \mathbb{Z}^{d}}\right\|_{\tilde{f}_{p, q}^{s, \bar{\alpha}}} \\
& \quad:=\left\|\left(\sum_{\bar{j} \in \mathbb{N}_{0}^{d}} 2^{\left\|\left(j_{1} / \alpha_{1}, \ldots, j_{d} / \alpha_{d}\right)\right\|_{\infty} s q}\left|\sum_{\bar{k} \in \mathbb{Z}^{d}} \lambda_{\bar{j}, \bar{k}} \chi_{\bar{j}, \bar{k}}(\cdot)\right|^{q}\right)^{1 / q}\right\|_{p} \text { is finite. }
\end{aligned}
$$

(ii) If $0<p \leq \infty$ we define the sequence space $\tilde{b}_{p, q}^{s, \bar{\alpha}}$ as the collection of all sequences $\left(\lambda_{\bar{j}, \bar{k}}\right)_{\bar{j} \in \mathbb{N}_{0}^{d}, \bar{k} \in \mathbb{Z}^{d}} \subset \mathbb{C}$ such that the (quasi-)norm (usual modification in case $q=\infty$ )

$$
\begin{aligned}
& \left\|\left(\lambda_{\bar{j}, \bar{k}}\right)_{\bar{j} \in \mathbb{N}_{0}^{d}, \bar{k} \in \mathbb{Z}^{d}}\right\|_{\tilde{b}_{p, q}^{s, \bar{\alpha}}} \\
& :=\left(\sum_{\bar{j} \in \mathbb{N}_{0}^{d}} 2^{\left\|\left(j_{1} / \alpha_{1}, \ldots, j_{d} / \alpha_{d}\right)\right\|_{\infty} s q}\left\|\sum_{\bar{k} \in \mathbb{Z}^{d}} \lambda_{\bar{j}, \bar{k}} \chi_{\bar{j}, \bar{k}}(\cdot)\right\|_{p}^{q}\right)^{1 / q} \text { is finite. }
\end{aligned}
$$

Now we are ready to state the wavelet characterization of the space $\widetilde{F}_{p, q}^{s, \bar{\alpha}}\left(\mathbb{R}^{d}\right)$. Recall that for $0<p, q \leq \infty$ we put

$$
\sigma_{p, q}:=\max \{1 / p-1,1 / q-1,0\} \text { and } \sigma_{p}:=\max \{1 / p-1,0\}
$$

Remark 4.5 The theorem below states the result for the $F$-scale of spaces $\widetilde{F}_{p, q}^{a, \bar{\alpha}}\left(\mathbb{R}^{d}\right)$. As for the corresponding result for the Besov type spaces $\widetilde{B}_{p, q}^{s, \bar{\alpha}}\left(\mathbb{R}^{d}\right)$, we simply replace condition (11) on $K, L$ by

$$
K, L>\sigma_{p}+|s| / \alpha_{\min }
$$

and use the corresponding sequence spaces $\tilde{b}_{p, q}^{s, \bar{\alpha}}$. 
Theorem 4.6 Let $0<p<\infty, 0<q \leq \infty, s \in \mathbb{R}, \bar{\alpha}=\left(\alpha_{1}, \ldots, \alpha_{d}\right)>0$ with $\sum_{i=1}^{d} \alpha_{i}=d$. Let further $\psi_{0}, \psi$ be wavelets satisfying $(K)$ and $(L)$ above with

$$
K, L>\sigma_{p, q}+|s| / \alpha_{\min } .
$$

Then any $f \in \mathcal{S}^{\prime}\left(\mathbb{R}^{d}\right)$ belongs to $\widetilde{F}_{p, q}^{s, \bar{\alpha}}\left(\mathbb{R}^{d}\right)$ if and only if it can be represented as

$$
f=\sum_{\bar{j} \in \mathbb{N}_{0}^{d}} \sum_{\bar{k} \in \mathbb{Z}^{d}} \lambda_{\bar{j}, \bar{k}} \psi_{\bar{j}, \bar{k}}
$$

with $\left(\lambda_{\bar{j}, \bar{k}}\right)_{\bar{j}, \bar{k}} \in \tilde{f}_{p, q}^{s, \bar{\alpha}}$ and the sum converging in $\mathcal{S}^{\prime}\left(\mathbb{R}^{d}\right)$ with respect to some ordering. For each $f \in \widetilde{F}_{p, q}^{s, \bar{\alpha}}\left(\mathbb{R}^{d}\right)$ the convergence of the representation (12) is then even unconditional. Moreover, if $q<\infty$, the sum also converges in $\widetilde{F}_{p, q}^{s, \bar{\alpha}}\left(\mathbb{R}^{d}\right)$ and $\left(\psi_{\bar{j}, \bar{k}}\right)_{\bar{j}, \bar{k}}$ constitutes an unconditional basis in $\widetilde{F}_{p, q}^{s, \bar{\alpha}}\left(\mathbb{R}^{d}\right)$. The sequence of coefficients $\lambda(f):=$ $(\lambda \bar{j}, \bar{k})_{\bar{j}, \bar{k}}$ is uniquely determined via

$$
\lambda_{\bar{j}, \bar{k}}=2^{\|\bar{j}\|_{1}}\left\langle f, \psi_{\bar{j}, \bar{k}}\right\rangle
$$

and we have the wavelet isomorphism (equivalent (quasi-)norm)

$$
\|f\|_{\widetilde{F}_{p, q}^{s, \bar{\alpha}}\left(\mathbb{R}^{d}\right)} \asymp\|\lambda(f)\|_{\tilde{f}_{p, q}^{s, \bar{\alpha}}}, \quad f \in \widetilde{F}_{p, q}^{s, \bar{\alpha}}\left(\mathbb{R}^{d}\right) .
$$

Remark 4.7 Following [34, Prop. 3.20], the dual pairing of $f \in \mathcal{S}^{\prime}\left(\mathbb{R}^{d}\right)$ and $\psi_{\bar{j}, \bar{k}} \in$ $C^{K}\left(\mathbb{R}^{d}\right)$ in (13) has to be understood in the way (for a justification see Corollary 4.10 below)

$$
\langle f, \psi\rangle:=\sum_{\bar{j} \in \mathbb{N}_{0}^{d}}\left\langle\Theta_{\bar{j}} * f, \Lambda_{\bar{j}} * \psi\right\rangle_{L_{2}\left(\mathbb{R}^{d}\right)} .
$$

Here we choose $\Theta_{\bar{j}}:=\mathcal{F}^{-1} \theta_{\bar{j}}$ and $\Lambda_{\bar{j}}:=\mathcal{F}^{-1} \lambda_{\bar{j}}$ such that $\sum_{\bar{j} \in \mathbb{N}_{0}^{d}} \theta_{\bar{j}} \lambda_{\bar{j}} \equiv 1$ (as e.g. in (28)). Using elementary estimates and the Nikol'skij inequality in case $p<1$, one can show

$$
\widetilde{F}_{p, q}^{s, \bar{\alpha}}\left(\mathbb{R}^{d}\right) \hookrightarrow \widetilde{B}_{p, \infty}^{s, \bar{\alpha}}\left(\mathbb{R}^{d}\right) \hookrightarrow S_{\max \{p, 1\}, \infty}^{-|s| / \alpha_{\min }-\sigma_{p}} B\left(\mathbb{R}^{d}\right)
$$

Setting $s_{\bar{\alpha}, p}:=|s| / \alpha_{\min }+\sigma_{p}$ and $\tilde{p}:=\max \{p, 1\}$ we obtain

$$
\begin{aligned}
|\langle f, \psi\rangle| & \leq \sum_{\bar{j} \in \mathbb{N}_{0}^{d}}\left\|\Theta_{\bar{j}} * f\right\|_{\tilde{p}}\left\|\Lambda_{\bar{j}} * \psi\right\|_{\tilde{p}^{\prime}} \\
& \leq \sup _{\bar{j} \in \mathbb{N}_{0}^{d}} 2^{-s_{\bar{\alpha}, p}\|\bar{j}\|_{1}}\left\|\Theta_{\bar{j}} * f\right\|_{\tilde{p}} \cdot \sum_{\bar{j} \in \mathbb{N}_{0}^{d}} 2^{s_{\bar{\alpha}, p}\|\bar{j}\|_{1}}\left\|\Lambda_{\bar{j}} * \psi\right\|_{\tilde{p}^{\prime}} \\
& \lesssim\|f\|_{S_{\tilde{p}, \infty}^{-s \bar{\alpha}, p} B\left(\mathbb{R}^{d}\right)} \cdot\|\psi\|_{S_{\tilde{p}^{\prime}, 1}^{s \bar{\alpha}, p} B\left(\mathbb{R}^{d}\right)},
\end{aligned}
$$


where the right-hand side is finite due to (11) and (9). In other words, $f \in \widetilde{F}_{p, q}^{s, \bar{\alpha}}\left(\mathbb{R}^{d}\right)$ generates a (conjugate) linear functional on the Banach space $S_{\tilde{p}^{\prime}, 1}^{s_{\bar{\alpha}}} B\left(\mathbb{R}^{d}\right)$.

Remark 4.8 As we will see below, our arguments apply as well to classical spaces of dominating mixed smoothness $S_{p, q}^{r} B\left(\mathbb{R}^{d}\right)$ and $S_{p, q}^{r} F\left(\mathbb{R}^{d}\right)$, defined in Definition 3.7 above. Examining the proof, we obtain for the relation

$$
\|f\|_{S_{p, q}^{r} F\left(\mathbb{R}^{d}\right)} \lesssim\|\lambda(f)\|_{s_{p, q}^{r} f}
$$

where $s_{p, q}^{r} f$ is the sequence space associated to $S_{p, q}^{r} F$ (for a definition see [59, Def. 2.1]), the condition

$$
L>\sigma_{p, q}-r \text { and } K>r
$$

The converse relation holds under the condition

$$
K>\sigma_{p, q}-r \text { and } L>r .
$$

For the spaces $S_{p, q}^{r} B\left(\mathbb{R}^{d}\right)$ we replace $\sigma_{p, q}$ by $\sigma_{p}$ and $s_{p, q}^{r} f$ by $s_{p, q}^{r} b$, which is the sequence space associated to $S_{p, q}^{r} B$ (for a definition see [59, Def. 2.1]).

Proof of Theorem 4.6 Step 1. We consider the sum

$$
f:=\sum_{\bar{j} \in \mathbb{N}_{0}^{d}} \sum_{\bar{k} \in \mathbb{Z}^{d}} \lambda_{\bar{j}, \bar{k}} \psi_{\bar{j}, \bar{k}}
$$

with $\lambda:=\left(\lambda_{\bar{j}, \bar{k}}\right)_{\bar{j}, \bar{k}} \in \tilde{f}_{p, q}^{s, \bar{\alpha}}$ and show the relation

$$
\|f\|_{\widetilde{F}_{p, q}^{s, \bar{\alpha}}\left(\mathbb{R}^{d}\right)} \lesssim\|\lambda\|_{\tilde{f}_{p, q}^{s, \bar{\alpha}}}
$$

For the issues on the convergence and uniqueness of (16) and (13) we refer to Step 3 and 4 below, where we show that under the assumption $\left(\lambda_{\bar{j}, \bar{k}}\right)_{\bar{j}, \bar{k}} \in \tilde{f}_{p, q}^{s, \bar{\alpha}}$ the element $f$ is well defined, with unconditional convergence of (16) at least in $\mathcal{S}^{\prime}\left(\mathbb{R}^{d}\right)$, which is sufficient for the subsequent considerations.

Let us consider $\Delta_{\bar{j}} f$ for some chosen hyperbolic Littlewood-Paley analysis. This gives for fixed $\bar{j} \in \mathbb{N}_{0}^{d}$

$$
2^{\|\bar{j} / \bar{\alpha}\|_{\infty} s}\left|\Delta_{\bar{j}} f(x)\right| \leq \sum_{\bar{\ell} \in \mathbb{Z}^{d}} 2^{\|\bar{j} / \bar{\alpha}\|_{\infty} s}\left|\Theta_{\bar{j}} *\left(\sum_{\bar{k} \in \mathbb{Z}^{d}} \lambda_{\bar{j}+\bar{\ell}, \bar{k}} \psi_{\bar{j}+\bar{\ell}, \bar{k}}\right)(x)\right|,
$$


where $\lambda_{\bar{j}+\bar{\ell}, \bar{k}}:=0$ whenever $j_{i}+\ell_{i}<0$ for at least one index $i \in\{1, \ldots, d\}$, $\Theta_{\bar{j}}:=\mathcal{F}^{-1} \theta_{\bar{j}}$ and $\left(\theta_{\bar{j}}\right)_{\bar{j}}$ is the system from Definition 3.2. With $u:=\min \{p, q, 1\}$

$$
\begin{aligned}
\|f\|_{\widetilde{F}_{p, q}^{s, \bar{\alpha}}\left(\mathbb{R}^{d}\right)} & =\left\|2^{\|\bar{j} / \bar{\alpha}\|_{\infty} s} \Delta_{\bar{j}} f(\cdot)\right\|_{L_{p}\left(\ell_{q}\right)} \\
& \lesssim\left(\sum_{\bar{\ell} \in \mathbb{Z}^{d}}\left\|2^{\|\bar{j} / \bar{\alpha}\|_{\infty} s} \Theta_{\bar{j}} *\left(\sum_{\bar{k} \in \mathbb{Z}^{d}} \lambda_{\bar{j}+\bar{\ell}, \bar{k}} \psi_{\bar{j}+\bar{\ell}, \bar{k}}\right)(\cdot)\right\|_{L_{p}\left(\ell_{q}[\bar{j}]\right)}^{u}\right)^{1 / u} .
\end{aligned}
$$

With the help of Lemma 4.2 we are aiming for pointwise estimates first.

$$
\begin{aligned}
& \left|\Theta_{\bar{j}} *\left(\sum_{\bar{k} \in \mathbb{Z}^{d}} \lambda_{\bar{j}+\bar{\ell}, \bar{k}} \psi_{\bar{j}+\bar{\ell}, \bar{k}}\right)(x)\right| \\
& \quad \leq \sum_{\bar{k} \in \mathbb{Z}^{d}}\left|\lambda_{\bar{j}+\bar{\ell}, \bar{k}}\right| \cdot\left|\left(\Theta_{\bar{j}} * \psi_{\bar{j}+\bar{\ell}, \bar{k}}\right)(x)\right| \\
& \quad \lesssim\left(\prod_{i=1}^{d} 2^{-N_{\operatorname{sign}\left(\ell_{i}\right)}\left|\ell_{i}\right|}\right) \sum_{\bar{k} \in \mathbb{Z}^{d}}\left|\lambda_{\bar{j}+\bar{\ell}, \bar{k}}\right| \prod_{i=1}^{d}\left(1+2^{\min \left\{j_{i}, j_{i}+\ell_{i}\right\}}\left|x_{i}-2^{-\left(j_{i}+\ell_{i}\right)} k_{i}\right|\right)^{-R}
\end{aligned}
$$

where we choose $R>1 / r$ with $r<\min \{1, p, q\}=u$. Note that, due to condition $(K)$ for the wavelet and Lemma 4.2, we can choose $R>0$ arbitrarily large. This allows for estimating with the help of Proposition 4.3

$$
\begin{aligned}
& \left|\Theta_{\bar{j}} *\left(\sum_{\bar{k} \in \mathbb{Z}^{d}} \lambda_{\bar{j}+\bar{\ell}, \bar{k}} \psi_{\bar{j}+\bar{\ell}, \bar{k}}\right)(x)\right| \\
& \quad \lesssim 2^{-\left\langle\bar{N}_{\operatorname{sign}(\bar{\ell})}, \bar{\ell} \mid\right\rangle^{\left\|\bar{\ell}_{+}\right\|_{1} / r}\left[M_{[d]}\left|\sum_{\bar{k} \in \mathbb{Z}^{d}} \lambda_{\bar{j}+\bar{\ell}, \bar{k}} \chi_{\bar{j}+\bar{\ell}, \bar{k}}(\cdot)\right|^{r}\right]^{1 / r}(x) .}
\end{aligned}
$$

Hereby we use the short-hand notation $\bar{\ell}_{+}:=\left(\left(\ell_{1}\right)_{+}, \ldots,\left(\ell_{d}\right)_{+}\right)$and

$$
\left\langle\bar{N}_{\operatorname{sign}(\bar{\ell})},|\bar{\ell}|\right\rangle:=\sum_{i=1}^{d} N_{\operatorname{sign}\left(\ell_{i}\right)}\left|\ell_{i}\right|
$$

Plugging this estimate into (17) gives

$$
\begin{aligned}
\|f\|_{\widetilde{F}_{p, q}^{s, \bar{\alpha}}\left(\mathbb{R}^{d}\right)} \lesssim & \left(\sum_{\bar{\ell} \in \mathbb{Z}^{d}} 2^{-u\left\langle\bar{N}_{\operatorname{sign}(\bar{\ell})},|\bar{\ell}|\right\rangle} 2^{u\left\|\bar{\ell}_{+}\right\|_{1} / r}\right. \\
& \| 2^{-\|(\bar{j}+\bar{\ell}) / \bar{\alpha}\|_{\infty} s} 2^{\|\bar{j} / \bar{\alpha}\|_{\infty} s}\left[M_{[d]} \mid 2^{\|(\bar{j}+\bar{\ell}) / \bar{\alpha}\|_{\infty} s}\right. \\
& \left.\left.\sum_{\bar{k} \in \mathbb{Z}^{d}} \lambda_{\bar{j}+\bar{\ell}, \bar{k}} \chi_{\bar{j}+\bar{\ell},\left.\bar{k}\right|^{r}}\right]^{1 / r}(\cdot) \|_{L_{p}\left(\ell_{q}[\bar{j}]\right)}^{u}\right)^{\frac{1}{u}}
\end{aligned}
$$


Clearly, if $s \geq 0$ then $\|(\bar{j}+\bar{\ell}) / \bar{\alpha}\|_{\infty} s \geq\|\bar{j} / \bar{\alpha}\|_{\infty} s-\|\bar{\ell} / \bar{\alpha}\|_{\infty} s$ and hence

$$
2^{-\|(\bar{j}+\bar{\ell}) / \bar{\alpha}\|_{\infty} s} 2^{\|\bar{j} / \bar{\alpha}\|_{\infty} s} \leq 2^{\|\bar{\ell} / \bar{\alpha}\|_{\infty}|s|} .
$$

In addition, if $s<0$ we also obtain (18) via the usual $\ell_{\infty}$-triangle inequality.

Putting this into the previous estimate, using the vector-valued Hardy-Littlewood maximal inequality (Theorem 2.4 and Remark 2.5) for the space $L_{p / r}\left(\ell_{q / r}[\bar{j}]\right)$, we obtain

$$
\begin{aligned}
\|f\|_{\widetilde{F}_{p, q}^{s, \bar{\alpha}}\left(\mathbb{R}^{d}\right)} & \left(\sum_{\bar{\ell} \in \mathbb{Z}^{d}} 2^{-u\left\langle\bar{N}_{\operatorname{sign}(\bar{\ell})},|\bar{\ell}|\right\rangle} 2^{u\left\|\bar{\ell}_{+}\right\|_{1} / r} 2^{u\|\bar{\ell} / \bar{\alpha}\|_{\infty}|s|}\right. \\
& \left.\left\|2^{\|(\bar{j}+\bar{\ell}) / \bar{\alpha}\|_{\infty} s}\left|\sum_{\bar{k} \in \mathbb{Z}^{d}} \lambda_{\bar{j}+\bar{\ell}, \bar{k}} \chi_{\bar{j}+\bar{\ell}, \bar{k} \mid}\right|\right\|_{L_{p}\left(\ell_{q}[\bar{j}]\right)}^{u}\right)^{1 / u} \\
& \lesssim\|\lambda\|_{\tilde{f}_{p, q}^{s, \bar{\alpha}}}\left(\sum_{\bar{\ell} \in \mathbb{Z}^{d}} 2^{-u\left\langle\bar{N}_{\operatorname{sign}(\bar{\ell})}, \bar{\ell} \mid\right\rangle} 2^{u\left\|\bar{\ell}_{+}\right\|_{1} / r} 2^{u\|\bar{\ell} / \bar{\alpha}\|_{1}|s|}\right)^{1 / u}
\end{aligned}
$$

The sum over $\bar{\ell}$ converges if $L+1=N_{+}>1 / r+|s| / \alpha_{\min }$ and $K=N_{-}>|s| / \alpha_{\min }$.

Step 2. Let us prove the converse relation $\|\lambda(f)\|_{\tilde{f}_{p, q}^{s, \bar{\alpha}}} \lesssim\|f\|_{\widetilde{F}_{p, q}^{s, \bar{\alpha}}\left(\mathbb{R}^{d}\right)}$ with $\lambda(f)=\left(2^{\|\bar{j}\|_{1}}\left\langle f, \psi_{\bar{j}, \bar{k}}\right\rangle\right) \bar{j}_{\bar{j}, \bar{k}}$ and start with $f \in \widetilde{F}_{p, q}^{s, \bar{\alpha}}\left(\mathbb{R}^{d}\right)$. As already pointed out in Remark 4.7, the dual pairing $\left\langle f, \psi_{\bar{j}, \bar{k}}\right\rangle$ makes sense due to condition (11). Our estimation begins as follows,

$$
\begin{aligned}
\left|2^{\|\bar{j}\|_{1}}\left\langle f, \psi_{\bar{j}, \bar{k}}\right\rangle\right| & \leq \sum_{\bar{\ell} \in \mathbb{Z}^{d}}\left|2^{\|\bar{j}\|_{1}}\left\langle\Theta_{\bar{j}+\bar{\ell}} * f, \Lambda_{\bar{j}+\bar{\ell}} * \psi_{\bar{j}, \bar{k}}\right\rangle\right| \\
& \leq \sum_{\bar{\ell} \in \mathbb{Z}^{d}} \sum_{\bar{m} \in \mathbb{Z}^{d}}\left|\int_{Q_{\bar{j}+\bar{\ell}, \bar{m}}}\left(\Theta_{\bar{j}+\bar{\ell}} * f\right)(y) 2^{\|\bar{j}\|_{1}}\left(\Lambda_{\bar{j}+\bar{\ell}} * \psi_{\bar{j}, \bar{k}}\right)(y) d y\right| \\
& \leq \sum_{\bar{\ell} \in \mathbb{Z}^{d}} \sum_{\bar{m} \in \mathbb{Z}^{d}}\left|\theta_{\bar{j}+\bar{\ell}, \bar{m}}(f)\right| \int_{Q_{\bar{j}+\bar{\ell}, \bar{m}}} 2^{\|\bar{j}\|_{1}}\left|\left(\Lambda_{\bar{j}+\bar{\ell}} * \psi_{\bar{j}, \bar{k}}\right)(y)\right| d y,
\end{aligned}
$$

where $\Theta_{\bar{j}+\bar{\ell}}=\Lambda_{\bar{j}+\bar{\ell}}=0$ whenever $j_{i}+\ell_{i}<0$ for at least one index $i \in\{1, \ldots, d\}$ and

$$
\theta_{\bar{j}+\bar{\ell}, \bar{m}}(f):=\sup _{y \in Q_{\bar{j}+\bar{\ell}, \bar{m}}}\left|\left(\Theta_{\bar{j}+\bar{\ell}} * f\right)(y)\right| .
$$

We next estimate the integral, as in Step 1 with the help of Lemma 4.2. Here we have to be particularly careful with the normalization factors. Note that, compared to (9), the signs of the components of $\bar{\ell}$ in the convolution $\Lambda_{\bar{j}+\bar{\ell}} * \psi_{\bar{j}, \bar{k}}$ change the role. This is why this time the factor $2^{-\left\langle\bar{N}_{\text {sign }(-\bar{\ell})},|\bar{\ell}|\right\rangle}$ appears and why we put $M_{0}:=0$, 
$M_{-}:=L, M_{+}:=K+1$ and $\bar{M}_{\operatorname{sign}(\bar{\ell})}:=\left(M_{\operatorname{sign}\left(\ell_{1}\right)}, \ldots, M_{\operatorname{sign}\left(\ell_{d}\right)}\right)$. We then obtain for $z=\left(z_{1}, \ldots, z_{d}\right) \in Q_{\bar{j}, \bar{k}}$ the estimate

$$
\begin{aligned}
& \int_{Q_{\bar{j}+\bar{\ell}, \bar{m}}} 2^{\|\bar{j}\|_{1}} \mid\left(\Lambda_{\bar{j}+\bar{\ell}} * \psi \bar{j}, \bar{k}\right. \\
& \\
& \lesssim \sup _{y \in Q_{\bar{j}+\bar{\ell}, \bar{m}}} 2^{-\left\langle\bar{N}_{\operatorname{sign}(-\bar{\ell})},|\bar{\ell}|\right\rangle} \prod_{i=1}^{d} 2^{-\ell_{i}}\left(1+2^{\min \left\{j_{i}, j_{i}+\ell_{i}\right\}}\left|y_{i}-2^{-j_{i}} k_{i}\right|\right)^{-R} \\
& \lesssim 2^{-\left\langle\bar{N}_{\operatorname{sign}(-\bar{\ell})}+\operatorname{sgn}(\bar{\ell}),|\bar{\ell}|\right\rangle} \prod_{i=1}^{d}\left(1+2^{\min \left\{j_{i}, j_{i}+\ell_{i}\right\}}\left|2^{-\left(j_{i}+\ell_{i}\right)} m_{i}-2^{-j_{i}} k_{i}\right|\right)^{-R} \\
& \lesssim 2^{-\left\langle\bar{M}_{\operatorname{sign}(\bar{\ell})},|\bar{\ell}|\right\rangle} \prod_{i=1}^{d}\left(1+2^{\min \left\{j_{i}, j_{i}+\ell_{i}\right\}}\left|2^{-\left(j_{i}+\ell_{i}\right)} m_{i}-z_{i}\right|\right)^{-R} .
\end{aligned}
$$

This together with (19) and Proposition 4.3 yields for $r<\min \{p, q, 1\}=u$ and $z \in \mathbb{R}^{d}$

$$
\begin{aligned}
& \sum_{\bar{k} \in \mathbb{Z}^{d}}\left|2^{\|\bar{j}\|_{1}}\left\langle f, \psi_{\bar{j}, \bar{k}}\right\rangle\right| \chi_{\bar{j}, \bar{k}}(z) \\
& \quad \lesssim \sum_{\bar{\ell} \in \mathbb{Z}^{d}} 2^{-\left\langle\bar{M}_{\text {sign }(\bar{\ell})},|\bar{\ell}|\right\rangle} \sum_{\bar{m} \in \mathbb{Z}^{d}}\left|\theta_{\bar{j}+\bar{\ell}, \bar{m}}(f)\right| \prod_{i=1}^{d}\left(1+2^{\min \left\{j_{i}, j_{i}+\ell_{i}\right\}}\left|2^{-\left(j_{i}+\ell_{i}\right)} m_{i}-z_{i}\right|\right)^{-R} \\
& \lesssim \sum_{\bar{\ell} \in \mathbb{Z}^{d}} 2^{-\left\langle\bar{M}_{\text {sign }(\bar{\ell})},|\bar{\ell}|\right\rangle} 2^{\left\|\bar{\ell}_{+}\right\|_{1} / r}\left[M_{[d]}\left|\sum_{\bar{m} \in \mathbb{Z}^{d}} \theta_{\bar{j}+\bar{\ell}, \bar{m}}(f) \chi_{\bar{j}+\bar{\ell}, \bar{m}}(\cdot)\right|^{r}\right]^{1 / r}(z) .
\end{aligned}
$$

This leads to

$$
\begin{aligned}
2^{\|\bar{j} / \bar{\alpha}\|_{\infty} s} & \sum_{\bar{k} \in \mathbb{Z}^{d}}\left|2^{\|\bar{j}\|_{1}}\left\langle f, \psi_{\bar{j}, \bar{k}}\right\rangle\right| \chi_{\bar{j}, \bar{k}} \\
\lesssim & \sum_{\bar{\ell} \in \mathbb{Z}^{d}} 2^{\left\|\bar{\ell}_{+}\right\|_{1} / r-\left\langle\bar{M}_{\operatorname{sign}(\bar{\ell})}, \bar{\ell} \mid\right\rangle+\|\bar{j} / \bar{\alpha}\|_{\infty} s-\|(\bar{j}+\bar{\ell}) / \bar{\alpha}\|_{\infty} s}\left[M_{[d]} \mid 2^{\|(\bar{j}+\bar{\ell}) / \bar{\alpha}\|_{\infty}}\right. \\
& \left.\left.\sum_{\bar{m} \in \mathbb{Z}^{d}} \theta_{\bar{j}+\bar{\ell}, \bar{m}}(f) \chi_{\bar{j}+\bar{\ell}, \bar{m}}(\cdot)\right|^{r}\right]^{\frac{1}{r}}
\end{aligned}
$$


Taking the $L_{p}\left(\ell_{q}[\bar{j}]\right)$-(quasi-)norm on both sides and using (18) once more, we obtain

$$
\begin{aligned}
& \left\|2^{\|\bar{j} / \bar{\alpha}\|_{\infty} s} \sum_{\bar{k} \in \mathbb{Z}^{d}}\left|2^{\|\bar{j}\|_{1}}\left\langle f, \psi_{\bar{j}, \bar{k}}\right\rangle\right| \chi_{\bar{j}, \bar{k}}\right\|_{L_{p}\left(\ell_{q}[\bar{j}]\right)} \\
& \lesssim\left(\sum_{\bar{\ell} \in \mathbb{Z}^{d}} 2^{-u\left\langle\bar{M}_{\operatorname{sign}(\bar{\ell})},|\bar{\ell}|\right\rangle} 2^{u\left\|\bar{\ell}_{+}\right\|_{1} / r} 2^{u\|\bar{\ell} / \bar{\alpha}\|_{\infty}|s|} \times\right. \\
& \left.\quad \times\left\|\left[M_{[d]}\left|2^{\|(\bar{j}+\bar{\ell}) / \bar{\alpha}\|_{\infty} s} \sum_{\bar{m} \in \mathbb{Z}^{d}} \theta_{\bar{j}+\bar{\ell}, \bar{m}}(f) \chi_{\bar{j}+\bar{\ell}, \bar{m}}(\cdot)\right|^{r}\right]^{1 / r}\right\|_{L_{p}\left(\ell_{q}[\bar{j}]\right)}^{u}\right)^{1 / u} .
\end{aligned}
$$

Due to $r<\min \{1, p, q\}=u$ we can apply the vector-valued Hardy-Littlewood maximal inequality (Theorem 2.4 and Remark 2.5) and obtain

$$
\begin{aligned}
& \left\|\left[M_{[d]}\left|2^{\|(\bar{j}+\bar{\ell}) / \bar{\alpha}\|_{\infty} s} \sum_{\bar{m} \in \mathbb{Z}^{d}} \theta_{\bar{j}+\bar{\ell}, \bar{m}}(f) \chi_{\bar{j}+\bar{\ell}, \bar{m}}\right|^{r}\right]^{1 / r}\right\|_{L_{p}\left(\ell_{q}[\bar{j}]\right)} \\
& \quad \lesssim\left\|2^{\|\bar{j} / \bar{\alpha}\|_{\infty} s} \sum_{\bar{m} \in \mathbb{Z}^{d}} \theta_{\bar{j}, \bar{m}}(f) \chi_{\bar{j}, \bar{m}}\right\|_{L_{p}\left(\ell_{q}\right)} .
\end{aligned}
$$

From (20) we obtain for any $z \in Q_{\bar{j}, \bar{m}}$ and any $a>0$

$$
\left|\theta_{\bar{j}, \bar{m}}(f)\right|=\sup _{y \in Q_{\bar{j}, \bar{m}}}\left|\left(\Theta_{\bar{j}} * f\right)(y)\right| \lesssim \sup _{y \in \mathbb{R}^{d}} \frac{\left|\left(\Theta_{\bar{j}} * f\right)(y)\right|}{\prod_{i=1}^{d}\left(1+2^{j_{i}}\left|z_{i}-y_{i}\right|\right)^{a}}=P_{2^{j}, a}\left(\Theta_{\bar{j}} * f\right)(z)
$$

where we used the definition of the Peetre maximal function in (6). Choosing $a>$ $\max \left\{\frac{1}{p}, \frac{1}{q}\right\}$ with the corresponding maximal inequality in Theorem 2.7 then yields the relation

$$
\begin{aligned}
\left\|2^{\|\bar{j} / \bar{\alpha}\|_{\infty} s} \sum_{\bar{m} \in \mathbb{Z}^{d}} \theta_{\bar{j}, \bar{m}}(f) \chi_{\bar{j}, \bar{m}}\right\|_{L_{p}\left(\ell_{q}\right)} & \lesssim\left\|P_{2^{j}, a}\left(2^{\|\bar{j} / \bar{\alpha}\|_{\infty} s} \Theta_{\bar{j}} * f\right)\right\|_{L_{p}\left(\ell_{q}\right)} \\
& \lesssim\left\|2^{\|\bar{j} / \bar{\alpha}\|_{\infty} s} \Theta_{\bar{j}} * f\right\|_{L_{p}\left(\ell_{q}\right)} \asymp\|f\|_{\widetilde{F}_{p, q}^{s, \bar{\alpha}}\left(\mathbb{R}^{d}\right)} .
\end{aligned}
$$

Returning to (21), we have seen

$$
\begin{aligned}
& \left\|2^{\|\bar{j} / \bar{\alpha}\|_{\infty} s} \sum_{\bar{k} \in \mathbb{Z}^{d}}\left|2^{\|\bar{j}\|_{1}}\left\langle f, \psi_{\bar{j}, \bar{k}}\right\rangle\right| \chi_{\bar{j}, \bar{k}}\right\|_{L_{p}\left(\ell_{q}[\bar{j}]\right)} \\
& \quad \lesssim\|f\|_{\left.\widetilde{F}_{p}^{s, q}, \mathbb{R}^{d}\right)}\left(\sum_{\bar{\ell} \in \mathbb{Z}^{d}} 2^{-u\left\langle\bar{M}_{\operatorname{sign}(\bar{\ell})},|\bar{\ell}|\right\rangle} 2^{u\left\|\bar{\ell}_{+}\right\|_{1} / r} 2^{u\|\bar{\ell} / \bar{\alpha}\|_{\infty}|s|}\right)^{1 / u} .
\end{aligned}
$$

It remains to discuss the sum over $\bar{\ell}$. It is easy to see that it converges if $K+1=$ $M_{+}>1 / r+|s| / \alpha_{\min }$ and $L=M_{-}>|s| / \alpha_{\min }$. Recall that $r$ is chosen such that $r<\min \{1, p, q\}$. 
Step 3. Let us now clarify the convergence issues in (12) in case $q<\infty$. The arguments in Step 1 above show in particular for a finite partial summation of (12) that

$$
\left\|\sum_{\bar{j}} \sum_{\bar{k}} \lambda_{\bar{j}, \bar{k}} \psi_{\bar{j}, \bar{k}}\right\|_{\widetilde{F}_{p, q}^{s, \bar{\alpha}}\left(\mathbb{R}^{d}\right)} \lesssim\left\|\left(\sum_{\bar{j}} 2^{\|\bar{j} / \bar{\alpha}\|_{\infty} s q}\left|\sum_{\bar{k}} \lambda_{\bar{j}, \bar{k}} \chi_{\bar{j}, \bar{k}}\right|^{q}\right)^{1 / q}\right\|_{p} .
$$

If $q<\infty$ (note that $p<\infty$ anyway) we use Lebesgue's dominated convergence theorem to conclude the unconditional convergence of $(12)$ in $\widetilde{F}_{p, q}^{s, \bar{\alpha}}\left(\mathbb{R}^{d}\right)$. The required majorant is thereby given by

$$
\left\|\left(\lambda_{\bar{j}, \bar{k}}\right)_{\bar{j} \in \mathbb{N}_{0}^{d}, \bar{k} \in \mathbb{Z}^{d}}\right\|_{\tilde{f}_{p, q}^{s, \bar{\alpha}}}<\infty .
$$

In case $q=\infty$ we use the observation in Remark 4.8. From a simple application of Hölder's inequality (with respect to the sum over $\bar{j}$ ) we first obtain for any $\varepsilon>0$ the relation

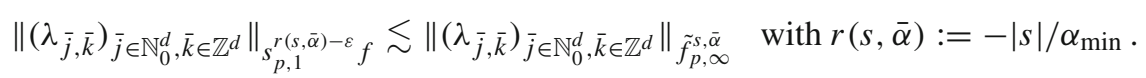

Choosing $\varepsilon>0$ small enough, we then obtain from (11) that condition (15) in Remark 4.8 is satisfied. Hence, for a finite partial summation of (12) we have

$$
\left\|\sum_{\bar{j}} \sum_{\bar{k}} \lambda_{\bar{j}, \bar{k}} \psi_{\bar{j}, \bar{k}}\right\|_{S_{p, 1}^{r(s, \alpha)-\varepsilon} F\left(\mathbb{R}^{d}\right)} \lesssim\left\|\sum_{\bar{j}} 2^{(r(s, \bar{\alpha})-\varepsilon)\|\bar{j}\|_{1}} \sum_{\bar{k}}\left|\lambda_{\bar{j}, \bar{k}}\right| \chi_{\bar{j}, \bar{k}}\right\|_{p} .
$$

Again, by Lebesgue's dominated convergence theorem (the majorant given by (22)) we see the unconditional convergence of (12) in the space $S_{p, 1}^{r(s, \alpha)-\varepsilon} F\left(\mathbb{R}^{d}\right)$. Taking the embedding $S_{p, 1}^{r(s, \alpha)-\varepsilon} F\left(\mathbb{R}^{d}\right) \hookrightarrow \mathcal{S}^{\prime}\left(\mathbb{R}^{d}\right)$ into account, we actually proved more than stated in the theorem.

Step 4. It remains to prove (12) for $f \in \widetilde{F}_{p, q}^{s, \bar{\alpha}}\left(\mathbb{R}^{d}\right)$ and coefficients $\lambda_{\bar{j}, \bar{k}}(f)=$ $2^{\|\bar{j}\|_{1}}\left\langle f, \psi_{\bar{j}, \bar{k}}\right\rangle$ chosen as in (13). From Steps 1, 2, 3 above we have learned that $\left\{\lambda_{\bar{j}, \bar{k}}(f)\right\}_{\bar{j}, \bar{k}} \in \tilde{f}_{p, q}^{s, \bar{\alpha}}$, which implies that the sum

$$
\sum_{\bar{j} \in \mathbb{N}_{0}^{d}} \sum_{\bar{k} \in \mathbb{Z}^{d}} \lambda_{\bar{j}, \bar{k}}(f) \psi_{\bar{j}, \bar{k}}
$$

converges (at least in) $\mathcal{S}^{\prime}\left(\mathbb{R}^{d}\right)$ to an element $g \in \mathcal{S}^{\prime}\left(\mathbb{R}^{d}\right)$. We now prove that $f(\varphi)=$ $g(\varphi)$ for all $\varphi \in \mathcal{S}\left(\mathbb{R}^{d}\right)$. Fix $\varphi \in \mathcal{S}\left(\mathbb{R}^{d}\right)$, then clearly $\bar{\varphi} \in L_{2}\left(\mathbb{R}^{d}\right)$ and we have

$$
\bar{\varphi}=\sum_{\bar{j} \in \mathbb{N}_{0}^{d}} \sum_{\bar{k} \in \mathbb{Z}^{d}} 2^{\|\bar{j}\|_{1}}\left\langle\bar{\varphi}, \psi_{\bar{j}, \bar{k}}\right\rangle \psi_{\bar{j}, \bar{k}}
$$


with convergence in $L_{2}\left(\mathbb{R}^{d}\right)$. Since $\bar{\varphi} \in S_{\tilde{p}^{\prime}, 1}^{S \bar{\alpha}, p} B\left(\mathbb{R}^{d}\right)$ we have by Step 1, 2, 3 above that the right-hand side of (23) converges in $S_{\tilde{p}^{\prime}, 1}^{s_{\bar{\alpha}, p}} B\left(\mathbb{R}^{d}\right)$ to some $\eta \in S_{\tilde{p}^{\prime}, 1}^{s_{\alpha}, p} B\left(\mathbb{R}^{d}\right)$. Hence, we have $\bar{\varphi}=\eta$ in $\mathcal{S}^{\prime}\left(\mathbb{R}^{d}\right)$ which finally gives $\bar{\varphi}=\eta$ almost everywhere and, in other words, $(23)$ holds true in $S_{\tilde{p}^{\prime}, 1}^{s \bar{\alpha},} B\left(\mathbb{R}^{d}\right)$. Then $f(\varphi)$ can be rewritten as follows, using Corollary 4.10 and the continuity of $\langle f, \cdot\rangle$ (see Remark 4.7),

$$
\begin{aligned}
f(\varphi)=\langle f, \bar{\varphi}\rangle & =\left\langle f, \sum_{\bar{j} \in \mathbb{N}_{0}^{d}} \sum_{\bar{k} \in \mathbb{Z}^{d}} 2^{\|\bar{j}\|_{1}}\left\langle\bar{\varphi}, \psi_{\bar{j}, \bar{k}}\right\rangle \psi_{\bar{j}, \bar{k}}\right\rangle \\
& =\sum_{\bar{j} \in \mathbb{N}_{0}^{d}} \sum_{\bar{k} \in \mathbb{Z}^{d}} 2^{\|\bar{j}\|_{1}} \overline{\left\langle\bar{\varphi}, \psi_{\bar{j}, \bar{k}}\right\rangle}\left\langle f, \psi_{\bar{j}, \bar{k}}\right\rangle \\
& =\sum_{\bar{j} \in \mathbb{N}_{0}^{d}} \sum_{\bar{k} \in \mathbb{Z}^{d}} 2^{\|\bar{j}\|_{1}}\left\langle\varphi, \overline{\psi_{\bar{j}, \bar{k}}}\right\rangle\left\langle f, \psi_{\bar{j}, \bar{k}}\right\rangle .
\end{aligned}
$$

On the other hand,

$$
g(\varphi)=\sum_{\bar{j} \in \mathbb{N}_{0}^{d}} \sum_{\bar{k} \in \mathbb{Z}^{d}} \lambda_{\bar{j}, \bar{k}}(f) \psi_{\bar{j}, \bar{k}}(\varphi)=\sum_{\bar{j} \in \mathbb{N}_{0}^{d}} \sum_{\bar{k} \in \mathbb{Z}^{d}} 2^{\|\bar{j}\|_{1}}\left\langle\varphi, \overline{\psi_{\bar{j}, \bar{k}}}\right\rangle\left\langle f, \psi_{\bar{j}, \bar{k}}\right\rangle=f(\varphi),
$$

which finishes the proof.

Lemma 4.9 Let $\left\{\varphi_{\bar{j}}\right\}_{\bar{j} \in \mathbb{N}_{0}^{d}}$ be a hyperbolic decomposition of unity (as defined in Sect. 3 ) and define $T_{\bar{j}}:=\sum_{\bar{k} \leq \bar{j}} \varphi_{\bar{k}}$. Then for any enumeration $\bar{j}=\bar{j}(N), N \in \mathbb{N}_{0}$, and every $\zeta \in \mathcal{S}\left(\mathbb{R}^{d}\right)$

$$
T_{\bar{j}(N)} \zeta \rightarrow \zeta \quad \text { (strongly) in } \mathcal{S}\left(\mathbb{R}^{d}\right) \text { as } N \rightarrow \infty
$$

Proof We first show (24) for the sequence $T_{N}:=\sum_{\|\bar{j}\|_{\infty} \leq N} \varphi_{\bar{j}}$, which is equivalent to showing $\left\|S_{N} \zeta\right\|_{k, \ell} \rightarrow 0$ for each fixed $k, \ell \in \mathbb{N}_{0}$ and $S_{N}:=1-T_{N}$. We estimate

$$
\left\|S_{N} \zeta\right\|_{k, \ell}=\left\|(1+|\cdot|)^{k} \sum_{\|\bar{\gamma}\|_{1} \leq \ell}\left|D^{\bar{\gamma}}\left(S_{N} \zeta\right)\right|\right\|_{\infty} \leq \sum_{\|\bar{\gamma}\|_{1} \leq \ell}\left\|(1+|\cdot|)^{k}\left|D^{\bar{\gamma}}\left(S_{N} \zeta\right)\right|\right\|_{\infty}
$$

and apply the multivariate Leibniz formula

$$
D^{\bar{\gamma}}\left(S_{N} \zeta(\cdot)\right)=\sum_{\bar{\nu} \leq \bar{\gamma}}\left(\begin{array}{l}
\bar{\gamma} \\
\bar{v}
\end{array}\right)\left(D^{\bar{\gamma}-\bar{v}} S_{N}\right)\left(D^{\bar{v}} \zeta\right)
$$

This leads to

$$
\left\|(1+|\cdot|)^{k}\left|D^{\bar{\gamma}}\left(S_{N} \zeta\right)\right|\right\|_{\infty} \leq \sum_{\bar{\nu} \leq \bar{\gamma}}\left(\begin{array}{l}
\bar{\gamma} \\
\bar{v}
\end{array}\right)\left\|(1+|\cdot|)^{k}\left(D^{\bar{\gamma}-\bar{v}} S_{N}\right)\left(D^{\bar{\nu}} \zeta\right)\right\|_{\infty}
$$


Since $\zeta \in \mathcal{S}\left(\mathbb{R}^{d}\right)$, there is a constant $C>0$ such that for every $x \in \mathbb{R}^{d}$ and every $\|\bar{v}\|_{1} \leq \ell$

$$
(1+|x|)^{k}\left|D^{\bar{v}} \zeta(x)\right| \leq C(1+|x|)^{-1} .
$$

In addition, due to $S_{N}=S_{0}\left(2^{-N} \cdot\right)$, we have $D^{\bar{\nu}} S_{N}(x)=2^{-N\|\bar{v}\|_{1}}\left(D^{\bar{\nu}} S_{0}\right)\left(2^{-N} x\right)$. Thus $\left\|D^{\bar{\nu}} S_{N}\right\|_{\infty} \leq 2^{-N\|\bar{v}\|_{1}} C^{\prime} \leq C^{\prime}$ with $C^{\prime}:=\max _{\|\bar{v}\|_{1} \leq \ell}\left\|D^{\bar{\nu}} S_{0}\right\|_{\infty}$. Using the fact that $D^{\bar{\gamma}-\bar{v}} S_{N}$ vanishes on the rectangle $R_{N}^{(1, \ldots, 1)}$ defined in (3), we can finally estimate for every $\bar{\gamma}, \bar{v} \in \mathbb{N}_{0}^{d}$ with $\bar{v} \leq \bar{\gamma}$ and $\|\bar{\gamma}\|_{1} \leq \ell$

$$
\begin{aligned}
& \left\|(1+|\cdot|)^{k}\left(D^{\bar{\gamma}-\bar{v}} S_{N}\right)\left(D^{\bar{\nu}} \zeta\right)\right\|_{\infty} \\
& \lesssim \sup _{x \in \mathbb{R}^{d} \backslash R_{N}^{(1, \ldots, 1)}}\left|(1+|x|)^{k} D^{\bar{\nu}} \zeta(x)\right| \lesssim\left(1+\left|2^{N}\right|\right)^{-1} \lesssim 2^{-N},
\end{aligned}
$$

with a right-hand side that tends to 0 for $N \rightarrow \infty$. This proves (24) for the sequence $T_{N}$. We conclude the argument with the observation that for every fixed $M \in \mathbb{N}_{0}$ the terms $\left\|T_{\bar{j}(N)} \zeta-\zeta\right\|_{k, \ell}$ eventually, as $N \rightarrow \infty$, become smaller than $\left\|T_{M} \zeta-\zeta\right\|_{k, \ell}=$ $\left\|S_{M} \zeta\right\|_{k, \ell}$.

Corollary 4.10 Let $\langle\cdot, \cdot\rangle$ be given as in (14). For every $f \in \mathcal{S}^{\prime}\left(\mathbb{R}^{d}\right)$ and $\zeta \in \mathcal{S}\left(\mathbb{R}^{d}\right)$ we have

$$
\langle f, \zeta\rangle_{\mathcal{S}^{\prime} \times \mathcal{S}}=\langle f, \bar{\zeta}\rangle
$$

Proof As a consequence of Lemma 4.9, we have with unconditional convergence in $\mathcal{S}\left(\mathbb{R}^{d}\right)$

$$
\mathcal{F}^{-1} \zeta=\sum_{\bar{j} \in \mathbb{N}_{0}^{d}} \theta_{\bar{j}} \lambda_{\bar{j}} \mathcal{F}^{-1} \zeta \quad \text { or equivalently } \zeta=\sum_{\bar{j} \in \mathbb{N}_{0}^{d}} \overline{\Theta_{\bar{j}}} * \overline{\Lambda_{\bar{j}}} * \zeta
$$

where $\bar{\Theta}_{\bar{j}}=\Theta_{\bar{j}}(-\cdot)=\mathcal{F} \theta_{\bar{j}}, \bar{\Lambda}_{\bar{j}}=\Lambda_{\bar{j}}(-\cdot)=\mathcal{F} \lambda_{\bar{j}}$, and $\theta_{\bar{j}}, \lambda_{\bar{j}}$ are functions with $\sum_{\bar{j} \in \mathbb{N}_{0}^{d}} \theta_{\bar{j}} \lambda_{\bar{j}} \equiv 1$ as in (14). The relations $\bar{\Theta}_{\bar{j}}=\Theta_{\bar{j}}(-\cdot)$ and $\bar{\Lambda}_{\bar{j}}=\Lambda_{\bar{j}}(-\cdot)$ are due to the fact that $\theta_{\bar{j}}$ and $\lambda_{\bar{j}}$ are real-valued functions. Hence, we can conclude

$$
\begin{aligned}
\langle f, \bar{\zeta}\rangle & =\sum_{\bar{j} \in \mathbb{N}_{0}^{d}}\left\langle\Theta_{j} * f, \Lambda_{\bar{j}} * \bar{\zeta}\right\rangle_{L_{2}\left(\mathbb{R}^{d}\right)}=\sum_{\bar{j} \in \mathbb{N}_{0}^{d}}\left\langle\bar{\Theta}_{\bar{j}}(-\cdot) * f, \overline{\Lambda_{\bar{j}}} * \zeta\right\rangle_{\mathcal{S}^{\prime} \times \mathcal{S}} \\
& =\sum_{\bar{j} \in \mathbb{N}_{0}^{d}}\left\langle f, \bar{\Theta}_{\bar{j}} * \overline{\Lambda_{\bar{j}}} * \zeta\right\rangle_{\mathcal{S}^{\prime} \times \mathcal{S}}=\langle f, \zeta\rangle_{\mathcal{S}^{\prime} \times \mathcal{S}} .
\end{aligned}
$$




\section{Hyperbolic Haar Characterization}

We next utilize a hyperbolic Haar basis for the characterization of the spaces $\widetilde{B}_{p, q}^{s, \bar{\alpha}}\left(\mathbb{R}^{d}\right)$ and $\widetilde{F}_{p, q}^{s, \bar{\alpha}}\left(\mathbb{R}^{d}\right)$ from Definitions 3.3 and 3.4, the main result being Theorem 5.4. It will show that Haar characterizations are possible in a certain restricted range of parameters, although the Haar wavelet does not fulfill smoothness requirements $(K)$ as assumed for the derivation of Theorem 4.6 in the previous section. Hence, for the proof of Theorem 5.4 a different methodology is needed than in Sect. 4 . We follow the technique used in [?], exploiting the special structure of the Haar wavelet, and want to mention that this technique is presumably extendable to higher order spline systems enabling characterizations in a larger parameter range.

We begin by fixing a convenient inhomogeneous Haar system on the real line, namely

$$
\mathcal{H}_{1}:=\left\{h_{j, k}: k \in \mathbb{Z}, j \in \mathbb{N}_{0}\right\}
$$

where for $j \in \mathbb{N}, k \in \mathbb{Z}$, the functions $h_{j, k}$ are scaled Haar functions of the form

$$
h_{j, k}(x):=\frac{1}{\sqrt{2}} h\left(2^{j-1} x-k\right) \quad, \quad \text { where } h(x):=\mathbb{1}_{I_{0,0}^{+}}(x)-\mathbb{1}_{I_{0,0}^{-}}(x) .
$$

The intervals $I_{j, k}^{+}=\left[2^{-j} k, 2^{-j}(k+1 / 2)\right)$ and $I_{j, k}^{-}=\left[2^{-j}(k+1 / 2), 2^{-j}(k+\right.$ 1)) thereby represent the dyadic children of the standard dyadic intervals $I_{j, k}=$ $\left[2^{-j} k, 2^{-j}(k+1)\right)$. At the lowest scale $j=0$ the ordinary Haar functions $\mathbb{1}_{I_{0, k}^{+}}-\mathbb{1}_{I_{0, k}^{-}}$ are replaced by the characteristic functions $h_{0, k}:=\mathbb{1}_{I_{0, k}}$. Further, we set $h_{j, k} \equiv 0$ if $j<0$. Defined like this, the structure of the system $\mathcal{H}_{1}$ fits closely to the wavelet systems considered in Sect. 4. The inhomogeneous scale is at $j=0$ (and not the standard $j=-1$ for Haar systems).

For dimension $d \in \mathbb{N}$ we derive a corresponding hyperbolic $d$-variate Haar system by the following tensorization procedure,

$$
\begin{aligned}
\mathcal{H}_{d} & :=\left\{h_{\bar{j}, \bar{k}}:=h_{j_{1}, k_{1}} \otimes \cdots \otimes h_{j_{d}, k_{d}}: \bar{k}=\left(k_{1}, \ldots, k_{d}\right) \in \mathbb{Z}^{d},\right. \\
\bar{j} & \left.=\left(j_{1}, \ldots, j_{d}\right) \in \mathbb{N}_{0}^{d}\right\} .
\end{aligned}
$$

As already earlier in (8), we denote by $\chi_{\bar{j}, \bar{k}}$ the characteristic function of the rectangle

$$
Q_{\bar{j}, \bar{k}}:=\left[2^{-j_{1}} k_{1}, 2^{-j_{1}}\left(k_{1}+1\right)\right) \times \cdots \times\left[2^{-j_{d}} k_{d}, 2^{-j_{d}}\left(k_{d}+1\right)\right) .
$$

At each fixed "scale" $\bar{j}$ these rectangles represent a partition of the $d$-dimensional domain $\mathbb{R}^{d}$. For $\bar{j} \in \mathbb{N}_{0}^{d}$ and $\bar{k} \in \mathbb{Z}^{d}$ they moreover correspond to the strict support of the Haar functions, namely strict $\operatorname{supp}\left(h_{\bar{j}, \bar{k}}\right)=\left\{x \in \mathbb{R}^{d}: h_{\bar{j}, \bar{k}}(x) \neq 0\right\}=$ $Q_{\left(j_{1}-1, \ldots, j_{d}-1\right)_{+}, \bar{k}}$. 
Proposition 5.1 Let $1<p, q<\infty, s \in \mathbb{R}$, and $\bar{\alpha}=\left(\alpha_{1}, \ldots, \alpha_{d}\right)>0$ such that $\sum_{i=1}^{d} \alpha_{i}=d$. Under the condition

$$
|s| / \alpha_{\min }<\min \left\{1-\frac{1}{p}, 1-\frac{1}{q}\right\}
$$

we have for $f \in \mathcal{S}^{\prime}\left(\mathbb{R}^{d}\right.$ ) (with the dual pairing $\left\langle f, h_{\bar{j}, \bar{k}}\right\rangle$ defined as in (14) in Remark 4.7)

$$
\left\|\left(\sum_{\bar{j} \in \mathbb{N}_{0}^{d}} 2^{\|\bar{j} / \bar{\alpha}\|_{\infty} s q}\left|\sum_{\bar{k} \in \mathbb{Z}^{d}} 2^{\|\bar{j}\|_{1}}\left\langle f, h_{\bar{j}, \bar{k}}\right\rangle \chi_{\bar{j}, \bar{k}}(x)\right|^{q}\right)^{1 / q}\right\|_{p} \lesssim\|f\|_{\widetilde{F}_{p, q}^{s, \bar{\alpha}},},
$$

whenever the left-hand side is defined. In case $|s| / \alpha_{\min }<1-\frac{1}{p}$ we have

$$
\left(\sum_{\bar{j} \in \mathbb{N}_{0}^{d}} 2^{\|\bar{j} / \bar{\alpha}\|_{\infty} s q}\left\|\sum_{\bar{k} \in \mathbb{Z}^{d}} 2^{\|\bar{j}\|_{1}}\left\langle f, h_{\bar{j}, \bar{k}}\right\rangle \chi_{\bar{j}, \bar{k}}(\cdot)\right\|_{p}^{q}\right)^{1 / q} \lesssim\|f\|_{\widetilde{B}_{p, q}^{s, \bar{\alpha}}} .
$$

Proof For the proof, we first build a suitable decomposition of unity adapted to the hyperbolic tiling of the frequency domain. For a respective construction, we start with univariate functions $\phi_{0}, \phi \in \mathcal{S}(\mathbb{R})$ and $\lambda_{0}, \lambda \in \mathcal{S}(\mathbb{R})$ such that

$$
\lambda_{0} \phi_{0}+\sum_{j \in \mathbb{N}} \lambda\left(2^{-j} \cdot\right) \phi\left(2^{-j} \cdot\right)=\sum_{j \in \mathbb{N}_{0}} \lambda_{j} \phi_{j} \equiv 1
$$

where $\phi_{j}:=\phi\left(2^{-j} \cdot\right)$ and $\lambda_{j}:=\lambda\left(2^{-j}\right.$.) for $j \in \mathbb{N}$. The functions $\phi_{0}$ and $\phi_{1}$ shall thereby, as usual, be compactly supported with

$$
\operatorname{supp}\left(\phi_{0}\right) \subset\{|x| \leq 2 \varepsilon\} \quad, \quad \operatorname{supp}(\phi) \subset\{\varepsilon / 2 \leq|x| \leq 2 \varepsilon\}
$$

for some $\varepsilon>0$. As a consequence, their inverse Fourier transforms however, namely $\Phi_{0}:=\mathcal{F}^{-1} \phi_{0}$ and $\Phi:=\mathcal{F}^{-1} \phi$, cannot have compact supports.

The functions $\lambda_{0}, \lambda$, on the other hand are chosen such that the supports of $\Lambda_{0}:=$ $\mathcal{F}^{-1} \lambda_{0}$ and $\Lambda:=\mathcal{F}^{-1} \lambda$ are compact. Further, they are assumed to fulfill the Tauberian conditions

$$
\left|\lambda_{0}(x)\right|>0 \quad \text { on }\{|x| \leq 2 \varepsilon\} \quad, \quad|\lambda(x)|>0 \quad \text { on }\{\varepsilon / 2 \leq|x| \leq 2 \varepsilon\}
$$

with the same $\varepsilon>0$ as above and furthermore $\lambda(0)=\lambda^{\prime}(0)=0$. Such a construction is indeed possible, see [55, Lem. 3.6] for example.

For the subsequent proof, it is convenient to also define the functions $\Phi_{j}:=\mathcal{F}^{-1} \phi_{j}$ and $\Lambda_{j}:=\mathcal{F}^{-1} \lambda_{j}$ for $j \in \mathbb{N}$. They fulfill the scaling relations $\Phi_{j}=2^{j} \Phi\left(2^{j} \cdot\right)$ and $\Lambda_{j}=2^{j} \Lambda\left(2^{j} \cdot\right)$. 
Next, we put $\Phi_{j}:=0$ and $\Lambda_{j}:=0$ for $j \in \mathbb{Z}$ with $j<0$ and build the tensor products

$$
\Phi_{\bar{\ell}}:=\bigotimes_{i \in\{1, \ldots, d\}} \Phi_{\ell_{i}} \text { and } \Lambda_{\bar{\ell}}:=\bigotimes_{i \in\{1, \ldots, d\}} \Lambda_{\ell_{i}} \text { for } \bar{\ell} \in \mathbb{Z}^{d}
$$

Then we have the decomposition, which in fact is a discrete version of Calderón's reproducing formula,

$$
f=\sum_{\bar{\ell} \in \mathbb{Z}^{d}} \Phi_{\bar{\ell}} * \Lambda_{\bar{\ell}} * f \text { for every } f \in \mathcal{S}^{\prime}\left(\mathbb{R}^{d}\right)
$$

enabling a component-wise evaluation of the scalar product $\left\langle f, h_{\bar{j}, \bar{k}}\right\rangle$. Each Haar coefficient can in this way be understood in the following sense (see also Remark 4.7),

$$
\left\langle f, h_{\bar{j}, \bar{k}}\right\rangle=\sum_{\bar{\ell} \in \mathbb{Z}^{d}}\left\langle\Phi_{\bar{\ell}} * \Lambda_{\bar{\ell}} * f, h_{\bar{j}, \bar{k}}\right\rangle=\sum_{\bar{\ell} \in \mathbb{Z}^{d}}\left\langle\Phi_{\bar{\ell}} * f, \Lambda_{\bar{\ell}}(-\cdot) * h_{\bar{j}, \bar{k}}\right\rangle,
$$

whenever the right-hand sum converges.

If we further assume that $\Lambda_{\bar{\ell}}$ is even, we arrive at the estimate

$$
\left|2^{\|\bar{j}\|_{1}}\left\langle f, h_{\bar{j}, \bar{k}}\right\rangle\right| \lesssim \sum_{\bar{\ell} \in \mathbb{Z}^{d}}\left|\int_{\mathbb{R}^{d}} 2^{\|\bar{j}\|_{1}}\left(\Phi_{\bar{j}+\bar{\ell}} * f\right)(y)\left(\Lambda_{\bar{j}+\bar{\ell}} * h_{\bar{j}, \bar{k}}\right)(y) d y\right| \cdot
$$

Let us investigate the integral on the right-hand side, and for this let us define

$$
S_{\bar{j}, \bar{k}, \bar{\ell}}:=\operatorname{supp}\left(\Lambda_{\bar{j}+\bar{\ell}} * h_{\bar{j}, \bar{k}}\right)
$$

If $\min _{i \in[d]}\left\{j_{i}+\ell_{i}\right\}<0$ we have $S_{\bar{j}, \bar{k}, \bar{\ell}}=\emptyset$ and the integral vanishes. Otherwise, when $\min _{i \in[d]}\left\{j_{i}+\ell_{i}\right\} \geq 0$, we fix $a>0$ and $x \in \mathbb{R}^{d}$ and obtain the estimate

$$
\begin{aligned}
& \left|\int_{\mathbb{R}^{d}} 2^{\|\bar{j}\|_{1}}\left(\Phi_{\bar{j}+\bar{\ell}} * f\right)(y)\left(\Lambda_{\bar{j}+\bar{\ell}} * h_{\bar{j}, \bar{k}}\right)(y) d y\right| \\
& \leq P_{2^{\bar{j}+\bar{\ell}}, a}\left(\Phi_{\bar{j}+\bar{\ell}} * f\right)(x) \cdot \sup _{y \in S_{\bar{j}, \bar{k}, \bar{\ell}}}\left[\prod_{i=1}^{d}\left(1+2^{j_{i}+\ell_{i}}\left|x_{i}-y_{i}\right|\right)^{a}\right] \\
& \quad \cdot\left|\int_{\mathbb{R}^{d}} 2^{\|\bar{j}\|_{1}}\left(\Lambda_{\bar{j}+\bar{\ell}} * h_{\bar{j}, \bar{k}}\right)(z) d z\right|
\end{aligned}
$$

where $P_{2^{j}+\bar{\ell}, a}\left(\Phi_{\bar{j}+\bar{\ell}} * f\right)$ denotes the Peetre maximal function (see (6))

$$
P_{2^{\bar{j}+\bar{\ell}}, a}\left(\Phi_{\bar{j}+\bar{\ell}} * f\right)(x)=\sup _{y \in \mathbb{R}^{d}} \frac{\left|\left(\Phi_{\bar{j}+\bar{\ell}} * f\right)(y)\right|}{\left(1+2^{j_{1}+\ell_{1}}\left|x_{1}-y_{1}\right|\right)^{a} \cdots\left(1+2^{j_{d}+\ell_{d}}\left|x_{d}-y_{d}\right|\right)^{a}} .
$$


The integral term splits into

$$
\begin{aligned}
& \int_{\mathbb{R}^{d}} 2^{\|\bar{j}\|_{1}}\left(\Lambda_{\bar{j}+\bar{\ell}} * h_{\bar{j}, \bar{k}}\right)(z) d z \\
& \quad=2^{\|\bar{j}\|_{1}} \int_{\mathbb{R}}\left(\Lambda_{j_{1}+\ell_{1}} * h_{j_{1}, k_{1}}\right)(t) d t \cdots \int_{\mathbb{R}}\left(\Lambda_{j_{d}+\ell_{d}} * h_{j_{d}, k_{d}}\right)(t) d t
\end{aligned}
$$

according to the relation

$$
\Lambda_{\bar{j}+\bar{\ell}} * h_{\bar{j}, \bar{k}}=\left(\Lambda_{j_{1}+\ell_{1}} * h_{j_{1}, k_{1}}\right) \otimes \cdots \otimes\left(\Lambda_{j_{d}+\ell_{d}} * h_{j_{d}, k_{d}}\right)
$$

For fixed $i \in\{1, \ldots, d\}$, assuming $\ell_{i}<0$, we can then further estimate

$$
\int_{\mathbb{R}}\left(\Lambda_{j_{i}+\ell_{i}} * h_{j_{i}, k_{i}}\right)(y) d y \lesssim 2^{-j_{i}+\ell_{i}}
$$

since $\left|\operatorname{supp}\left(\Lambda_{j_{i}+\ell_{i}} * h_{j_{i}, k_{i}}\right)\right| \asymp 2^{-\left(j_{i}+\ell_{i}\right)}$ and $\left\|\Lambda_{j_{i}+\ell_{i}} * h_{j_{i}, k_{i}}\right\|_{\infty} \lesssim 2^{2 \ell_{i}}$. For the latter of these two inequalities the first order vanishing moment of the Haar wavelet comes into play. Note here that indeed $j_{i}>0$ due to $\min _{i \in[d]}\left\{j_{i}+\ell_{i}\right\} \geq 0$, allowing for the estimate

$$
\begin{aligned}
\left|\left(\Lambda_{j_{i}+\ell_{i}} * h_{j_{i}, k_{i}}\right)(x)\right| & \leq \int_{\operatorname{supp}\left(h_{j_{i}, k_{i}}\right)}\left|\Lambda_{j_{i}+\ell_{i}}(x-y)-\Lambda_{j_{i}+\ell_{i}}\left(x-2^{1-j_{i}} k_{i}\right)\right| \cdot\left|h_{j_{i}, k_{i}}(y)\right| d y \\
& \leq \sup _{y \in 2^{1-j_{i}}\left[k_{i}, k_{i}+1\right]}\left|\Lambda_{j_{i}+\ell_{i}}(x-y)-\Lambda_{j_{i}+\ell_{i}}\left(x-2^{1-j_{i}} k_{i}\right)\right| \cdot\left\|h_{j_{i}, k_{i}}\right\|_{1} \\
& \lesssim 2^{2\left(1-j_{i}\right)}\left\|\Lambda_{j_{i}+\ell_{i}}^{\prime}\right\|_{\infty} \lesssim 2^{2\left(1-j_{i}\right)} 2^{2\left(j_{i}+\ell_{i}\right)}\left\|\Lambda^{\prime}\right\|_{\infty} \lesssim 2^{2 \ell_{i}} .
\end{aligned}
$$

In case $\ell_{i} \geq 0$ we obtain a different estimate than (29), namely

$$
\int_{\mathbb{R}}\left(\Lambda_{j_{i}+\ell_{i}} * h_{j_{i}, k_{i}}\right)(y) d y \lesssim 2^{-\left(j_{i}+\ell_{i}\right)}
$$

Here we use the fact that the integrand is bounded by a constant together with the observation that its support is contained in at most three intervals of length $\asymp 2^{-\left(j_{i}+\ell_{i}\right)}$. Indeed, as a consequence of the $L_{1}$ - resp. $L_{\infty}$-normalization of $\Lambda_{j_{i}+\ell_{i}}$ and $h_{j_{i}, k_{i}}$, we have $\left\|\Lambda_{j_{i}+\ell_{i}} * h_{j_{i}, k_{i}}\right\|_{\infty} \leq\left\|\Lambda_{j_{i}+\ell_{i}}\right\|_{1}\left\|h_{j_{i}, k_{i}}\right\|_{\infty} \lesssim 1$. Furthermore, due to the vanishing moment properties of $\Lambda_{j_{i}+\ell_{i}}$, the support of the convolution merely stems from the either two or three discontinuities of the function $h_{j_{i}, k_{i}}$.

Now, let us turn our attention to the factor

$$
\sup _{y \in S_{\bar{j}, \bar{k}, \bar{\ell}}}\left[\prod_{i=1}^{d}\left(1+2^{j_{i}+\ell_{i}}\left|x_{i}-y_{i}\right|\right)^{a}\right] .
$$

Here, we have with $x_{i} \in Q_{j_{i}, k_{i}}$ and $y_{i} \in \operatorname{supp}\left(\Lambda_{j_{i}+\ell_{i}} * h_{j_{i}, k_{i}}\right) \subset \operatorname{supp}\left(\Lambda_{j_{i}+\ell_{i}}\right)+$ $\operatorname{supp}\left(h_{j_{i}, k_{i}}\right)$ (and therefore $\left|x_{i}-y_{i}\right| \lesssim 2^{-j_{i}}$ if $\ell_{i} \geq 0$ and $\left|x_{i}-y_{i}\right| \lesssim 2^{-\left(j_{i}+\ell_{i}\right)}$ if 
$\left.\ell_{i}<0\right)$

$$
\left(1+2^{j_{i}+\ell_{i}}\left|x_{i}-y_{i}\right|\right)^{a} \lesssim 1
$$

if $\ell_{i}<0$. Otherwise, if $\ell_{i} \geq 0$, we estimate

$$
\left(1+2^{j_{i}+\ell_{i}}\left|x_{i}-y_{i}\right|\right)^{a} \lesssim 2^{\ell_{i} a} .
$$

Putting all together, this yields for $x \in Q_{\bar{j}, \bar{k}}$

$$
\left|\int_{\mathbb{R}^{d}} 2^{\|\bar{j}\|_{1}}\left(\Phi_{\bar{j}+\bar{\ell}} * f\right)(y)\left(\Lambda_{\bar{j}+\bar{\ell}} * h_{\bar{j}, \bar{k}}\right)(y) d y\right| \lesssim A(\bar{\ell}, a) P_{2^{\bar{j}+\bar{\ell}}, a}\left(\Phi_{\bar{j}+\bar{\ell}} * f\right)(x),
$$

where

$$
A(\bar{\ell}, a):=\prod_{i \in\{1, \ldots, d\}} A\left(\ell_{i}, a\right) \quad \text { with } A\left(\ell_{i}, a\right):=\left\{\begin{array}{ll}
2^{\ell_{i}} & , \ell_{i}<0 \\
2^{(a-1) \ell_{i}} & , \ell_{i} \geq 0
\end{array} .\right.
$$

Hence, we obtain uniformly in $x \in \mathbb{R}^{d}$ and for fixed $\bar{j} \in \mathbb{N}_{0}^{d}$

$$
\sum_{\bar{k} \in \mathbb{Z}^{d}}\left|2^{\|\bar{j}\|_{1}}\left\langle f, h_{\bar{j}, \bar{k}}\right\rangle\right| \chi_{\bar{j}, \bar{k}}(x) \lesssim \sum_{\bar{\ell} \in \mathbb{Z}^{d}} A(\bar{\ell}, a) P_{2^{j}+\bar{\ell}, a}\left(\Phi_{\bar{j}+\bar{\ell}} * f\right)(x) .
$$

Finally, we can turn to the proof of (26). We estimate

$$
\begin{aligned}
& \left\|\left(\sum_{\bar{j} \in \mathbb{N}_{0}^{d}} 2^{\|\bar{j} / \bar{\alpha}\|_{\infty} s q}\left|\sum_{\bar{k} \in \mathbb{Z}^{d}} 2^{\|\bar{j}\|_{1}}\left\langle f, h_{\bar{j}, \bar{k}}\right\rangle \chi_{\bar{j}, \bar{k}}(x)\right|^{q}\right)^{1 / q}\right\|_{p} \\
& \lesssim\left\|\left(\sum_{\bar{j} \in \mathbb{N}_{0}^{d}} 2^{\|\bar{j} / \bar{\alpha}\|_{\infty} s q}\left|\sum_{\bar{\ell} \in \mathbb{Z}^{d}} A(\bar{\ell}, a) P_{2^{j}+\bar{\ell}, a}\left(\Phi_{\bar{j}+\bar{\ell}} * f\right)(x)\right|^{q}\right)^{1 / q}\right\|_{p} \\
& =\left\|\left(\sum_{\bar{j} \in \mathbb{N}_{0}^{d}}\left|\sum_{\bar{\ell} \in \mathbb{Z}^{d}} A(\bar{\ell}, a) 2^{\left(\|\bar{j} / \bar{\alpha}\|_{\infty}-\|(\bar{j}+\bar{\ell}) / \bar{\alpha}\|_{\infty}\right) s} 2^{\|(\bar{j}+\bar{\ell}) / \bar{\alpha}\|_{\infty} s} P_{2^{j}+\bar{\ell}, a}\left(\Phi_{\bar{j}+\bar{\ell}} * f\right)(x)\right|^{q}\right)^{1 / q}\right\|_{p} .
\end{aligned}
$$

According to (18) it holds $2^{\left(\|\bar{j} / \bar{\alpha}\|_{\infty}-\|(\bar{j}+\bar{\ell}) / \bar{\alpha}\|_{\infty}\right) s} \leq 2^{\|\bar{\ell} / \bar{\alpha}\|_{\infty}|s|}$ for $s \in \mathbb{R}$, and hence

$$
\begin{aligned}
& \left\|\left(\sum_{\bar{j} \in \mathbb{N}_{0}^{d}} 2^{\|\bar{j} / \bar{\alpha}\|_{\infty} s q}\left|\sum_{\bar{k} \in \mathbb{Z}^{d}} 2^{\|\bar{j}\|_{1}}\left\langle f, h_{\bar{j}, \bar{k}}\right\rangle \chi_{\bar{j}, \bar{k}}(x)\right|^{q}\right)^{1 / q}\right\|_{p} \\
& \leq\left\|\left(\sum_{\bar{j} \in \mathbb{N}_{0}^{d}}\left|\sum_{\bar{\ell} \in \mathbb{Z}^{d}} A(\bar{\ell}, a) 2^{\|\bar{\ell} / \bar{\alpha}\|_{\infty}|s|} 2^{\|(\bar{j}+\bar{\ell}) / \bar{\alpha}\|_{\infty} s} P_{2^{\bar{j}+\bar{\ell}}, a}\left(\Phi_{\bar{j}+\bar{\ell}} * f\right)(x)\right|^{q}\right)^{1 / q}\right\|_{p} \\
& \leq \sum_{\bar{\ell} \in \mathbb{Z}^{d}} A(\bar{\ell}, a) 2^{\|\bar{\ell} / \bar{\alpha}\|_{\infty}|s|} \cdot\left\|\left(\sum_{\bar{j} \in \mathbb{N}_{0}^{d}}\left|2^{\|\bar{j} / \bar{\alpha}\|_{\infty} s} P_{2^{\bar{j}}, a}\left(\Phi_{\bar{j}} * f\right)(x)\right|^{q}\right)^{1 / q}\right\|_{p},
\end{aligned}
$$


where Young's convolution inequality was used in the last step.

For (27) we argue analogously, namely

$$
\begin{aligned}
& \left(\sum_{\bar{j} \in \mathbb{N}_{0}^{d}} 2^{\|\bar{j} / \bar{\alpha}\|_{\infty} s q}\left\|\sum_{\bar{k} \in \mathbb{Z}^{d}} 2^{\|\bar{j}\|_{1}}\left\langle f, h_{\bar{j}, \bar{k}}\right\rangle \chi_{\bar{j}, \bar{k}}(\cdot)\right\|_{p}^{q}\right)^{1 / q} \\
& \lesssim\left(\sum_{\bar{j} \in \mathbb{N}_{0}^{d}} 2^{\|\bar{j} / \bar{\alpha}\|_{\infty} s q}\left\|\sum_{\bar{\ell} \in \mathbb{Z}^{d}} A(\bar{\ell}, a) P_{2^{j}+\bar{\ell}, a}\left(\Phi_{\bar{j}+\bar{\ell}} * f\right)(\cdot)\right\|_{p}^{q}\right)^{1 / q} \\
& \leq\left(\sum_{\bar{j} \in \mathbb{N}_{0}^{d}}\left\|\sum_{\bar{\ell} \in \mathbb{Z}^{d}} A(\bar{\ell}, a) 2^{\|\bar{\ell} / \bar{\alpha}\|_{\infty}|s|} 2^{\|(\bar{j}+\bar{\ell}) / \bar{\alpha}\|_{\infty} s} P_{2^{\bar{j}+\bar{\ell}, a}}\left(\Phi_{\bar{j}+\bar{\ell}} * f\right)(\cdot)\right\|_{p}^{q}\right)^{1 / q} \\
& \leq \sum_{\bar{\ell} \in \mathbb{Z}^{d}} A(\bar{\ell}, a) 2^{\|\bar{\ell} / \bar{\alpha}\|_{\infty}|s|} \cdot\left(\sum_{\bar{j} \in \mathbb{N}_{0}^{d}}\left\|2^{\|\bar{j} / \bar{\alpha}\|_{\infty} s} P_{2^{j}, a}\left(\Phi_{\bar{j}} * f\right)(\cdot)\right\|_{p}^{q}\right)^{1 / q} .
\end{aligned}
$$

Choosing $a>\max \{1 / p, 1 / q\}$ in the F-case and $a>1 / p$ in the B-case, to ensure the boundedness of the Peetre maximal operator (see Theorem 2.7, also compare e.g. [56, Thm. 2.6]), as well as $|s|<\min _{i \in\{1, \ldots, d\}}\left\{\alpha_{i}\right\}(1-a)$ we get

$$
\sum_{\bar{\ell} \in \mathbb{Z}^{d}} A(\bar{\ell}, a) 2^{\|\bar{\ell} / \bar{\alpha}\|_{\infty}|s|}<\infty
$$

and thus (26) and (27), respectively.

Using a duality argument, we can deduce an immediate companion result.

Proposition 5.2 Let $1<p, q<\infty, s \in \mathbb{R}$, and $\bar{\alpha}=\left(\alpha_{1}, \ldots, \alpha_{d}\right)>0$ such that $\sum_{i=1}^{d} \alpha_{i}=d$. Under the condition

$$
|s| / \alpha_{\min }<\min \left\{\frac{1}{p}, \frac{1}{q}\right\}
$$

we have for all $f \in \mathcal{S}^{\prime}\left(\mathbb{R}^{d}\right)$ (with the dual pairing $\left\langle f, h_{\bar{j}, \bar{k}}\right\rangle$ defined as in (14) in Remark 4.7)

$$
\|f\|_{\widetilde{F}_{p, q}^{s, \bar{\alpha}}} \lesssim\left\|\left(\sum_{\bar{j} \in \mathbb{N}_{0}^{d}} 2^{\|\bar{j} / \bar{\alpha}\|_{\infty} s q}\left|\sum_{\bar{k} \in \mathbb{Z}^{d}} 2^{\|\bar{j}\|_{1}}\left\langle f, h_{\bar{j}, \bar{k}}\right\rangle \chi_{\bar{j}, \bar{k}}(x)\right|^{q}\right)^{1 / q}\right\|_{p},
$$

whenever the right-hand side is defined. In case $|s| / \alpha_{\min }<1 / p$ we have

$$
\|f\|_{\widetilde{B}_{p, q}^{s, \bar{\alpha}}} \lesssim\left(\sum_{\bar{j} \in \mathbb{N}_{0}^{d}} 2^{\|\bar{j} / \bar{\alpha}\|_{\infty} s q}\left\|\sum_{\bar{k} \in \mathbb{Z}^{d}} 2^{\|\bar{j}\|_{1}}\left\langle f, h_{\bar{j}, \bar{k}}\right\rangle \chi_{\bar{j}, \bar{k}}(\cdot)\right\|_{p}^{q}\right)^{1 / q}
$$


Proof We showed in Proposition 5.1 (i) that the linear operator

$$
A: \widetilde{F}_{p, q}^{s, \bar{\alpha}} \rightarrow \tilde{f}_{p, q}^{s, \bar{\alpha}}, \quad f \mapsto\left(2^{\|\bar{j}\|_{1}}\left\langle f, h_{\bar{j}, \bar{k}}\right\rangle\right)_{\bar{j}, \bar{k}},
$$

is well-defined and bounded in the parameter range

$$
|s|<\min _{i \in\{1, \ldots, d\}}\left\{\alpha_{i}\right\} \min \left\{1-\frac{1}{p}, 1-\frac{1}{q}\right\} .
$$

Consequently, in this range, the dual operator

$$
A^{\prime}:\left(\tilde{f}_{p, q}^{s, \bar{\alpha}}\right)^{\prime} \rightarrow\left(\widetilde{F}_{p, q}^{s, \bar{\alpha}}\right)^{\prime}
$$

is also well-defined and bounded. Identifying $\left(\tilde{f}_{p, q}^{s, \bar{\alpha}}\right)^{\prime}$ with $\tilde{f}_{p^{\prime}, q^{\prime}}^{-s, \bar{\alpha}}$ with respect to the non-standard duality product

$$
\left\langle\left(\lambda_{\bar{j}, \bar{k}}\right),\left(\mu_{\bar{j}, \bar{k}}\right)\right\rangle:=\sum_{\bar{j} \in \mathbb{N}_{0}^{d}} 2^{-\|\bar{j}\|_{1}} \sum_{\bar{k} \in \mathbb{Z}^{d}} \lambda_{\bar{j}, \bar{k}} \mu_{\bar{j}, \bar{k}},
$$

which is possible according to Theorem 5.6 (i) below, it can be represented in the form

$$
A^{\prime}:\left(\lambda_{\bar{j}, \bar{k}}\right)_{\bar{j}, \bar{k}} \mapsto \sum_{\bar{j}, \bar{k}} \lambda_{\bar{j}, \bar{k}} h_{\bar{j}, \bar{k}}
$$

where the convergence is weak*ly in $\left(\widetilde{F}_{p, q}^{s, \bar{\alpha}}\right)^{\prime}$. This is a consequence of the relation

$$
\begin{aligned}
\left\langle A f, g^{\prime}\right\rangle_{Y \times Y^{\prime}} & =\sum_{\bar{j} \in \mathbb{N}_{0}^{d}} 2^{-\|\bar{j}\|_{1}} \sum_{\bar{k} \in \mathbb{Z}^{d}} 2^{\|\bar{j}\|_{1}}\left\langle f, h_{\bar{j}, \bar{k}}\right\rangle \cdot \lambda_{\bar{j}, \bar{k}} \\
& =\int_{\mathbb{R}^{d}} f(x) \cdot\left(\sum_{\bar{j} \in \mathbb{N}_{0}^{d}} \sum_{\bar{k} \in \mathbb{Z}^{d}} \lambda_{\bar{j}, \bar{k}} h_{\bar{j}, \bar{k}}(x)\right) d x \\
& =\left\langle f, A^{\prime} g^{\prime}\right\rangle_{X \times X^{\prime}},
\end{aligned}
$$

where we used the short-hand notation $Y=\tilde{f}_{p, q}^{s, \bar{\alpha}}$ and $X=\widetilde{F}_{p, q}^{s, \bar{\alpha}}$.

Invoking Theorem 5.6(i) another time, $X^{\prime}$ can be identified with $\widetilde{F}_{p^{\prime}, q^{\prime}}^{-s, \bar{\alpha}}$ (and $X^{\prime \prime}$ with $\left.\widetilde{F}_{p, q}^{s, \bar{\alpha}}\right)$. Then for $(\lambda \overline{j, \bar{k}})_{\bar{j}, \bar{k}} \in \tilde{f}_{p^{\prime}, q^{\prime}}^{-s, \bar{\alpha}}$ and some enumeration

$$
\left(\lambda_{\bar{j}, \bar{k}}\right)_{\bar{j}, \bar{k}} \rightsquigarrow\left(\lambda_{n}\right)_{n \in \mathbb{N}}
$$

we have $\left\|\left(\lambda_{n}\right)_{n \geq N}\right\|_{\tilde{f}_{p^{\prime}, q^{\prime}}^{-s, \bar{\alpha}}} \rightarrow 0$ for $N \rightarrow \infty$. We estimate

$$
\left\|A^{\prime}\left(\left(\lambda_{n}\right)_{n \geq N}\right)\right\|_{\widetilde{F}_{p^{\prime}, q^{\prime}}^{-s, \bar{\alpha}}}=\sup _{\|f\|_{\widetilde{F}_{p, q}^{s, \bar{\alpha}}=1}}\left|\left\langle A^{\prime}\left(\left(\lambda_{n}\right)_{n \geq N}\right), f\right\rangle\right|=\sup _{\|f\|_{\widetilde{F}_{p, q}^{s, \bar{\alpha}}=1}}\left|\left\langle\left(\lambda_{n}\right)_{n \geq N}, A f\right\rangle\right|
$$




$$
\begin{aligned}
& \leq \sup _{\|f\|_{\widetilde{F}_{p, q}^{s, \bar{\alpha}}=1}}\left\|\left(\lambda_{n}\right)_{n \geq N}\right\|_{\tilde{f}_{p^{\prime}, q^{\prime}}^{-s, \bar{\alpha}}\|A f\|_{\tilde{f}_{p, q}^{s, \bar{\alpha}}}} \\
& \lesssim\left\|\left(\lambda_{n}\right)_{n \geq N}\right\|_{\tilde{f}_{p^{\prime}, q^{\prime}}^{-s, \bar{\alpha}}} \rightarrow 0 \quad(N \rightarrow \infty) .
\end{aligned}
$$

Hence, $A^{\prime}\left(\left(\lambda_{n}\right)_{n \leq N}\right) \rightarrow A^{\prime}\left(\left(\lambda_{n}\right)_{n \in \mathbb{N}}\right)$ strongly and unconditionally in $\widetilde{F}_{p^{\prime}, q^{\prime}}^{-s, \bar{\alpha}}$.

In other words, we have shown that if $|s|<\min _{i \in\{1, \ldots, d\}}\left\{\alpha_{i}\right\} \min \{1 / p, 1 / q\}$ and $\left(\lambda_{\bar{j}, \bar{k}}\right)_{\bar{j}, \bar{k}} \in \tilde{f}_{p, q}^{s, \bar{\alpha}}$ then $\sum_{\bar{j}, \bar{k}} \lambda_{\bar{j}, \bar{k}} h_{\bar{j}, \bar{k}}$ converges strongly and unconditionally in $\widetilde{F}_{p, q}^{s, \bar{\alpha}}$ and

$$
\left\|\sum_{\bar{j}, \bar{k}} \lambda_{\bar{j}, \bar{k}} h_{\bar{j}, \bar{k}}\right\|_{\widetilde{F}_{p, q}^{s, \bar{\alpha}}} \lesssim\left\|\left(\lambda_{\bar{j}, \bar{k}}\right)_{\bar{j}, \bar{k}}\right\|_{\tilde{f}_{p, q}^{s, \bar{\alpha}}}
$$

Hence, choosing $\lambda_{\bar{j}, \bar{k}}:=2^{\|\bar{j}\|_{1}}\left\langle f, h_{\bar{j}, \bar{k}}\right\rangle$ and assuming a finite sequence norm

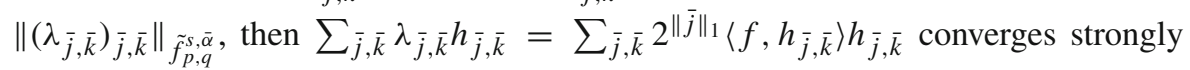
to some limit $\tilde{f} \in \widetilde{F}_{p, q}^{s, \bar{\alpha}}\left(\mathbb{R}^{d}\right)$. Since this sum also converges (weak*ly) in $S^{\prime}(\mathbb{R})$ to $f$ we have $f=\tilde{f}=\sum_{\bar{j}, \bar{k}} 2^{\|\bar{j}\|_{1}}\left\langle f, h_{\bar{j}, \bar{k}}\right\rangle h_{\bar{j}, \bar{k}}$ in $\widetilde{F}_{p, q}^{s, \bar{\alpha}}$. Now (30) follows from (33).

The proof of (31) works the same, using Proposition 5.1 (ii) and Theorem 5.6 (ii).

Combining both, Proposition 5.1 and Proposition 5.2, we arrive at the following proposition, whereby we now concentrate on the F-case.

Proposition 5.3 Let $1<p, q<\infty, s \in \mathbb{R}$, and $\bar{\alpha}=\left(\alpha_{1}, \ldots, \alpha_{d}\right)>0$ such that $\sum_{i=1}^{d} \alpha_{i}=d$. Under the condition

$$
|s| / \alpha_{\min }<\min \left\{\frac{1}{p}, \frac{1}{q}, 1-\frac{1}{p}, 1-\frac{1}{q}\right\}
$$

we have for all $f \in \mathcal{S}^{\prime}\left(\mathbb{R}^{d}\right)$ (with the dual pairing $\left\langle f, h_{\bar{j}, \bar{k}}\right\rangle$ defined as in (14) in Remark 4.7)

$$
\left\|\left(\sum_{\bar{j} \in \mathbb{N}_{0}^{d}} 2^{\|\bar{j} / \bar{\alpha}\|_{\infty} s q}\left|\sum_{\bar{k} \in \mathbb{Z}^{d}} 2^{\|\bar{j}\|_{1}}\left\langle f, h_{\bar{j}, \bar{k}}\right\rangle \chi_{\bar{j}, \bar{k}}(x)\right|^{q}\right)^{1 / q}\right\|_{p} \asymp\|f\|_{\widetilde{F}_{p, q}^{s, \bar{\alpha}}},
$$

whenever the left-hand side is defined.

As a direct consequence of this result, we can finally formulate the main theorem of this section which corresponds to Theorem 4.6. Let us remark that we expect a larger parameter range than (35) when moving to higher order spline systems (see e.g. Sect. 2.5 and Remark 2.14 in the book [53]) but do not pursue this path further in this article. 
Theorem 5.4 Let $1<p, q<\infty, s \in \mathbb{R}$, and $\bar{\alpha}=\left(\alpha_{1}, \ldots, \alpha_{d}\right)>0$ such that $\sum_{i=1}^{d} \alpha_{i}=d$. Further, assume

$$
|s| / \alpha_{\min }<\min \left\{\frac{1}{p}, \frac{1}{q}, 1-\frac{1}{p}, 1-\frac{1}{q}\right\} .
$$

Then the Haar system $\mathcal{H}_{d}=\left(h_{\bar{j}, \bar{k}}\right)_{\bar{j}, \bar{k}}$ defined in (25) constitutes an unconditional basis of $\widetilde{F}_{p, q}^{s, \bar{\alpha}}\left(\mathbb{R}^{d}\right)$ with associated sequence space $\tilde{f}_{p, q}^{s, \bar{\alpha}}$. The unique sequence of basis coefficients for $f \in \widetilde{F}_{p, q}^{s, \bar{\alpha}}\left(\mathbb{R}^{d}\right)$ is determined by $\lambda:=\lambda(f)=(\lambda \bar{j}, \bar{k})_{\bar{j}, \bar{k}}$ with

$$
\lambda_{\bar{j}, \bar{k}}:=\lambda_{\bar{j}, \bar{k}}(f)=2^{\|\bar{j}\|_{1}}\left\langle f, h_{\bar{j}, \bar{k}}\right\rangle .
$$

Further, we have the wavelet isomorphism (equivalent norm)

$$
\|f\|_{\widetilde{F}_{p, q}^{s, \bar{\alpha}}\left(\mathbb{R}^{d}\right)} \asymp\|\lambda(f)\|_{\tilde{f}_{p, q}^{s, \bar{\alpha}}}, \quad f \in \widetilde{F}_{p, q}^{s, \bar{\alpha}}\left(\mathbb{R}^{d}\right) .
$$

In addition, we can use $\mathcal{H}_{d}$ to distinguish those elements of $\mathcal{S}^{\prime}\left(\mathbb{R}^{d}\right)$ that belong to $\widetilde{F}_{p, q}^{s, \bar{\alpha}}\left(\mathbb{R}^{d}\right)$. Those are characterized by either of the following two criteria:

(i) $f$ can be represented as a sum

$$
f=\sum_{\bar{j} \in \mathbb{N}_{0}^{d}} \sum_{\bar{k} \in \mathbb{Z}^{d}} \lambda_{\bar{j}, \bar{k}} h_{\bar{j}, \bar{k}} \text { converging (weak } * \text { ly) in } \mathcal{S}^{\prime}\left(\mathbb{R}^{d}\right)
$$

with coefficients $\left(\lambda_{\bar{j}, \bar{k}}\right)_{\bar{j}, \bar{k}} \in \tilde{f}_{p, q}^{s, \bar{\alpha}}$ (with respect to some chosen ordering).

(ii) With $\lambda(f)$ being defined as in (36), it holds

$$
\lambda(f)=(\lambda \bar{j}, \bar{k}) \bar{j}, \bar{k} \in \tilde{f}_{p, q}^{s, \bar{\alpha}} .
$$

In both cases, the sequence $\left(\lambda_{\bar{j}, \bar{k}}\right)_{\bar{j}, \bar{k}}$ is necessarily the sequence of basis coefficients and the representation (37) converges unconditionally to $f$ in $\widetilde{F}_{p, q}^{s, \bar{\alpha}}\left(\mathbb{R}^{d}\right)$.

Proof As a direct consequence of the equivalence (34) proved in Proposition 5.3 the analysis operator

$$
\lambda: f \mapsto\left(2^{\|\bar{j}\|_{1}}\left\langle f, h_{\bar{j}, \bar{k}}\right\rangle\right)_{\bar{j}, \bar{k}}
$$

is well-defined and bounded from $\widetilde{F}_{p, q}^{s, \bar{\alpha}}$ to $\tilde{f}_{p, q}^{s, \bar{\alpha}}$. Moreover, it is injective and we have the equivalence of norms $\|f\|_{\widetilde{F}_{p, q}^{s, \bar{\alpha}}} \asymp\|\lambda(f)\|_{\tilde{f}_{p, q}^{s, \bar{\alpha}}}$. Further, for $f \in \mathcal{S}^{\prime}\left(\mathbb{R}^{d}\right)$ we have $f \in \widetilde{F}_{p, q}^{s, \bar{\alpha}}$ if and only if $\lambda(f) \in \tilde{f}_{p, q}^{s, \bar{\alpha}}$ (whenever $\lambda(f)$ is defined).

Now, let us have a look at the synthesis operator

$$
S:\left(\lambda_{\bar{j}, \bar{k}}\right)_{\bar{j}, \bar{k}} \mapsto \sum_{\bar{j}, \bar{k}} \lambda_{\bar{j}, \bar{k}} h_{\bar{j}, \bar{k}} .
$$


Clearly, for every finite sequence the assignment $S$ defines an element in $\widetilde{F}_{p, q}^{s, \bar{\alpha}}$. By completion, using (34) and the fact that the finite sequences lie dense in $\tilde{f}_{p, q}^{s, \bar{\alpha}}$, this synthesis further extends to all sequences of $\tilde{f}_{p, q}^{s, \bar{\alpha}}$, with unconditional and strong convergence of (38) in $\widetilde{F}_{p, q}^{s, \bar{\alpha}}$. Hence, $S$ is a well-defined bounded linear operator from $\tilde{f}_{p, q}^{s, \bar{\alpha}}$ to $\widetilde{F}_{p, q}^{s, \bar{\alpha}}$, which, as another consequence of (34), is also injective.

Next, turning to the composition $\lambda \circ S$ operating from $\tilde{f}_{p, q}^{s, \bar{\alpha}}$ to $\tilde{f}_{p, q}^{s, \bar{\alpha}}$, we deduce for each fixed $\bar{j}_{*} \in \mathbb{N}_{0}^{d}$ and $\bar{k}_{*} \in \mathbb{Z}^{d}$

$$
\left\langle\sum_{\bar{j}, \bar{k}} 2^{\|\bar{j}\|_{1}} \lambda_{\bar{j}, \bar{k}} h_{\bar{j}, \bar{k}}, h_{\bar{j}_{*}, \bar{k}_{*}}\right\rangle=\sum_{\bar{j}, \bar{k}} 2^{\|\bar{j}\|_{1}} \lambda_{\bar{j}, \bar{k}}\left\langle h_{\bar{j}, \bar{k}}, h_{\bar{j}_{*}, \bar{k}_{*}}\right\rangle=\lambda_{\bar{j}_{*}, \bar{k}_{*}},
$$

using the orthogonality of the system $\left(h_{\bar{j}, \bar{k}}\right)_{\bar{j}, \bar{k}}$ in $L_{2}\left(\mathbb{R}^{d}\right)$. We obtain $I d_{\tilde{f}_{p, q}^{s, \bar{\alpha}}}=\lambda \circ S$ and in turn $\lambda \circ S \circ \lambda=\lambda$. Due to the injectivity of $\lambda$, the latter equality further implies $I d_{\widetilde{F}_{p, q}^{s, \bar{\alpha}}}=S \circ \lambda$. In particular, $\lambda$ and $S$ are thus bijections and every $f \in \widetilde{F}_{p, q}^{s, \bar{\alpha}}$ allows for a representation (37).

To see that the representing coefficients $\left(\lambda_{\bar{j},}, \bar{k}\right) \bar{j}, \bar{k}$ are unique, under the assumption of strong convergence of the sum, let $\lambda^{*}=\left(\lambda \bar{j}_{\bar{j}, \bar{k}}^{*}\right) \bar{j}, \bar{k}$ be some sequence which satisfies (37) in a strong sense for some special ordering of the sum. Then again (34) together with a completion argument yields $\lambda^{*} \in \tilde{f}_{p, q}^{s, \bar{\alpha}}$, and thus $\lambda^{*}=\lambda(f)$ by the injectivity of $S$. Hence, the expansion coefficients in (37) are unique and it follows that $\mathcal{H}_{d}$ is a basis. Its unconditionality is due to the fact that the convergence of (37) for sequences $\lambda^{*} \in \tilde{f}_{p, q}^{s, \bar{\alpha}}$ is always unconditional.

For the proof of criterion (i) we just remark that for sequences $\left(\lambda_{\bar{j}, \bar{k}}\right)_{\bar{j}, \bar{k}}$ in $\tilde{f}_{p, q}^{s, \bar{\alpha}}$ with weak*-convergence of (37) in $\mathcal{S}^{\prime}\left(\mathbb{R}^{d}\right)$ the convergence is automatically in the stronger sense of $\widetilde{F}_{p, q}^{s, \bar{\alpha}}$.

Remark 5.5 For brevity, the above theorem was only stated for the F-case. There also exists a B-version, which reads precisely the same apart from condition (35) which is replaced by

$$
|s| / \alpha_{\min }<\min \left\{\frac{1}{p}, 1-\frac{1}{p}\right\} .
$$

In the proof of Proposition 5.2 we utilized isomorphisms $\left(\widetilde{F}_{p, q}^{s, \bar{\alpha}}\right)^{\prime} \cong \widetilde{F}_{p^{\prime}, q^{\prime}}^{-s, \bar{\alpha}}$ and $\left(\tilde{f}_{p, q}^{s, \bar{\alpha}}\right)^{\prime} \cong \tilde{f}_{p^{\prime}, q^{\prime}}^{-s, \bar{\alpha}}$ as well as $\left(\widetilde{B}_{p, q}^{s, \bar{\alpha}}\right)^{\prime} \cong \widetilde{B}_{p^{\prime}, q^{\prime}}^{-s, \bar{\alpha}}$ and $\left(\tilde{b}_{p, q}^{s, \bar{\alpha}}\right)^{\prime} \cong \tilde{b}_{p^{\prime}, q^{\prime}}^{-s, \bar{\alpha}}$. Hence, for the completeness of our exposition, it remains to establish those.

Theorem 5.6 Let $1<p, q<\infty, s \in \mathbb{R}$, and $\bar{\alpha}=\left(\alpha_{1}, \ldots, \alpha_{d}\right)>0$ with $\sum_{i=1}^{d} \alpha_{i}=$ d. Then

$$
\left(\widetilde{F}_{p, q}^{s, \bar{\alpha}}\left(\mathbb{R}^{d}\right)\right)^{\prime} \cong \widetilde{F}_{p^{\prime}, q^{\prime}}^{-s, \bar{\alpha}}\left(\mathbb{R}^{d}\right) \text { and }\left(\tilde{f}_{p, q}^{s, \bar{\alpha}}\right)^{\prime} \cong \tilde{f}_{p^{\prime}, q^{\prime}}^{-s, \bar{\alpha}}
$$


whereby the second of these isomorphies has to be understood with respect to the non-standard pairing (32). In the Besov case, we have the analogous relations

$$
\left(\widetilde{B}_{p, q}^{s, \bar{\alpha}}\left(\mathbb{R}^{d}\right)\right)^{\prime} \cong \widetilde{B}_{p^{\prime}, q^{\prime}}^{-s, \bar{\alpha}}\left(\mathbb{R}^{d}\right) \text { and }\left(\tilde{b}_{p, q}^{s, \bar{\alpha}}\right)^{\prime} \cong \tilde{b}_{p^{\prime}, q^{\prime}}^{-s, \bar{\alpha}}
$$

The proof of this theorem is based on two auxiliary propositions. The first one of these is stated without proof, since it is a straightforward generalization of the classic identifications $\left(L_{p}\left(\mathbb{R}^{d}\right)\right)^{\prime} \cong L_{p^{\prime}}\left(\mathbb{R}^{d}\right)$ and $\left(\ell_{p}(I)\right)^{\prime} \cong \ell_{p^{\prime}}(I)$ when $1<p<\infty$ (see e.g. [17]).

Proposition 5.7 Let I be an arbitrary countable index set. Then

$$
\left(\ell_{q}\left(I, L_{p}\left(\mathbb{R}^{d}\right)\right)\right)^{\prime} \cong \ell_{q^{\prime}}\left(I, L_{p^{\prime}}\left(\mathbb{R}^{d}\right)\right) \text { and }\left(L_{p}\left(\mathbb{R}^{d}, \ell_{q}(I)\right)\right)^{\prime} \cong L_{p^{\prime}}\left(\mathbb{R}^{d}, \ell_{q^{\prime}}(I)\right)
$$

in the sense that there exist isomorphisms $f \mapsto\left(f_{i}\right)_{i \in I}$ such that

$$
\langle f, g\rangle_{Y^{\prime} \times Y}=\sum_{i \in I} \int_{\mathbb{R}^{d}} f_{i}(x) g_{i}(x) d x
$$

for the respective cases $Y=\ell_{q}\left(I, L_{p}\left(\mathbb{R}^{d}\right)\right)$ and $Y=L_{p}\left(\mathbb{R}^{d}, \ell_{q}(I)\right)$.

The second proposition provides an alternative way to characterize functions in $\widetilde{F}_{p, q}^{s, \bar{\alpha}}\left(\mathbb{R}^{d}\right)$ and $\widetilde{B}_{p, q}^{s, \alpha}\left(\mathbb{R}^{d}\right)$. Its counterpart in the classical setting of Triebel-Lizorkin spaces is Proposition 1 in [52, Sect. 2.3.4].

Proposition 5.8 Assume $1<p, q<\infty, s \in \mathbb{R}, \bar{\alpha}=\left(\alpha_{1}, \ldots, \alpha_{d}\right)>0$, and $\sum_{i=1}^{d} \alpha_{i}=d$.

(i) Then $f \in \mathcal{S}^{\prime}\left(\mathbb{R}^{d}\right)$ belongs to $\widetilde{F}_{p, q}^{s, \bar{\alpha}}\left(\mathbb{R}^{d}\right)$ if and only if there exists a family $\left\{f_{\bar{j}}\right\}_{\bar{j} \in \mathbb{N}_{0}^{d}} \subset L_{p}\left(\mathbb{R}^{d}\right)$ such that

$$
f=\sum_{\bar{j}} \Delta_{\bar{j}} f_{\bar{j}} \text { in } \mathcal{S}^{\prime}\left(\mathbb{R}^{d}\right) \quad \text { and } \quad\left\|2^{\|\bar{j} / \bar{\alpha}\|_{\infty} s} f_{\bar{j}}\right\|_{L_{p}\left(\mathbb{R}^{d}, \ell_{q}\right)}<\infty .
$$

(ii) Then $f \in \mathcal{S}^{\prime}\left(\mathbb{R}^{d}\right)$ belongs to $\widetilde{B}_{p, q}^{s, \bar{\alpha}}\left(\mathbb{R}^{d}\right)$ if and only if there exists a family $\left\{f_{\bar{j}}\right\}_{\bar{j} \in \mathbb{N}_{0}^{d}} \subset L_{p}\left(\mathbb{R}^{d}\right)$ such that

$$
f=\sum_{\bar{j}} \Delta_{\bar{j}} f_{\bar{j}} \text { in } \mathcal{S}^{\prime}\left(\mathbb{R}^{d}\right) \quad \text { and } \quad\left\|2^{\|\bar{j} / \bar{\alpha}\|_{\infty} s} f_{\bar{j}}\right\|_{\ell_{q}\left(L_{p}\left(\mathbb{R}^{d}\right)\right)}<\infty
$$

Proof (i) Adaption of proof of Proposition 1 in [52, Sect. 2.3.4].

(ii) Let $\left(\phi_{j}\right)_{j}$ be a hyperbolic resolution of unity as introduced in Definition 3.2 with associated hyperbolic Littlewood-Paley analysis $\left(\Delta_{\bar{j}}\right)_{\bar{j}}$. Further, take $f \in \widetilde{B}_{p, q}^{s, \bar{\alpha}}$ 
and put $f_{\bar{j}}:=\sum_{\bar{r} \in\{-1,0,1\}^{d}} \mathcal{F}^{-1} \phi_{\bar{j}+\bar{r}} \mathcal{F} f$ for $\bar{j} \in \mathbb{N}_{0}^{d}$, whereby we let $\phi_{-1}:=0$. Then $\left\{f_{\bar{j}}\right\}_{\bar{j}} \subset L_{p}$ and

$$
\begin{aligned}
\sum_{\bar{j} \in \mathbb{N}_{0}^{d}} \mathcal{F}^{-1} \phi_{\bar{j}} \mathcal{F} f_{\bar{j}} & =\sum_{\bar{j} \in \mathbb{N}_{0}^{d}} \mathcal{F}^{-1} \phi_{\bar{j}}\left(\sum_{\bar{r} \in\{-1,0,1\}^{d}} \phi_{\bar{j}+\bar{r}}\right) \mathcal{F} f \\
& =\sum_{\bar{j} \in \mathbb{N}_{0}^{d}} \mathcal{F}^{-1} \phi_{\bar{j}} \mathcal{F} f=\sum_{\bar{j} \in \mathbb{N}_{0}^{d}} \Delta_{\bar{j}} f=f
\end{aligned}
$$

as well as

$$
\begin{aligned}
& \left\|2^{\|\bar{j} / \bar{\alpha}\|_{\infty} s} f_{\bar{j}}\right\|_{\ell_{q}\left(L_{p}\right)}=\left\|2^{\|\bar{j} / \bar{\alpha}\|_{\infty} s} \sum_{\bar{r} \in\{-1,0,1\}^{d}} \mathcal{F}^{-1} \phi_{\bar{j}+\bar{r}} \mathcal{F} f\right\|_{\ell_{q}\left(L_{p}\right)} \\
& \lesssim \sum_{\bar{r} \in\{-1,0,1\}^{d}}\left\|2^{\|\bar{j} / \bar{\alpha}\|_{\infty} s} \mathcal{F}^{-1} \phi_{\bar{j}+\bar{r}} \mathcal{F} f\right\|_{\ell_{q}\left(L_{p}\right)} \asymp\|f\|_{\widetilde{B}_{p, q}^{s, \bar{\alpha}}}<\infty .
\end{aligned}
$$

This settles one direction of the assertion. For the other direction, let $f \in \mathcal{S}^{\prime}\left(\mathbb{R}^{d}\right)$ satisfy (39) with associated $\left\{f_{\bar{j}}\right\}_{j} \subset L_{p}$. In view of $f=\sum_{\bar{j}} \Delta_{\bar{j}} f_{\bar{j}}$, we can estimate

$$
\begin{aligned}
\left\|2^{\|\bar{j} / \bar{\alpha}\|_{\infty} s} \Delta_{\bar{j}} f\right\|_{\ell_{q}\left(L_{p}\right)} & =\left\|2^{\|\bar{j} / \bar{\alpha}\|_{\infty} s} \Delta_{\bar{j}} \sum_{\bar{r} \in\{-1,0,1\}^{d}} \Delta_{\bar{j}+\bar{r}} f_{\bar{j}+\bar{r}}\right\|_{\ell_{q}\left(L_{p}\right)} \\
& \leq \sum_{\bar{r} \in\{-1,0,1\}^{d}}\left\|2^{\|\bar{j} / \bar{\alpha}\|_{\infty} s} \Delta_{\bar{j}} \Delta_{\bar{j}+\bar{r}} f_{\bar{j}+\bar{r}}\right\|_{\ell_{q}\left(L_{p}\right)} \\
& \lesssim \sum_{\bar{r} \in\{-1,0,1\}^{d}}\left\|2^{\|\bar{j} / \bar{\alpha}\|_{\infty} s} \Delta_{\bar{j}+\bar{r}} f_{\bar{j}+\bar{r}}\right\|_{\ell_{q}\left(L_{p}\right)} \\
& \asymp\left\|2^{\|\bar{j} / \bar{\alpha}\|_{\infty} s} \Delta_{\bar{j}} f_{\bar{j}}\right\|_{\ell_{q}\left(L_{p}\right)} \lesssim\left\|2^{\|\bar{j} / \bar{\alpha}\|_{\infty} s} f_{\bar{j}}\right\|_{\ell_{q}\left(L_{p}\right)}<\infty,
\end{aligned}
$$

where the last two lines are due to the Hörmander-Mikhlin multiplier theorem, which is applied twice. This estimate shows $f \in \widetilde{B}_{p, q}^{s, \bar{\alpha}}$, finishing the proof.

Now we are ready to give a thorough proof of the duality relations stated in Theorem 5.6.

Proof of Theorem 5.6 We restrict to the F-case and begin with the more involved relation $\left(\widetilde{F}_{p, q}^{s, \bar{\alpha}}\right)^{\prime} \cong \widetilde{F}_{p^{\prime}, q^{\prime}}^{-s, \bar{\alpha}}$. The subsequent proof is thereby an adaption of the proof of the classical theorem in [52, Sect. 2.11.2] to the setting of hyperbolic spaces.

It is essential to note that, since $\mathcal{S}\left(\mathbb{R}^{d}\right)$ lies dense in $\widetilde{F}_{p, q}^{s, \bar{\alpha}}$, there is a natural embedding

$$
\kappa:\left(\widetilde{F}_{p, q}^{s, \bar{\alpha}}\right)^{\prime} \hookrightarrow \mathcal{S}^{\prime}\left(\mathbb{R}^{d}\right) .
$$


Hence, both $\left(\widetilde{F}_{p, q}^{s, \bar{\alpha}}\right)^{\prime}$ and $\widetilde{F}_{p^{\prime}, q^{\prime}}^{-s, \bar{\alpha}}$ can be interpreted as subspaces of $\mathcal{S}^{\prime}\left(\mathbb{R}^{d}\right)$, simplifying the following considerations.

Let us first assume that $f \in \mathcal{S}^{\prime}\left(\mathbb{R}^{d}\right)$ is an element of $\widetilde{F}_{p^{\prime}, q^{\prime}}^{-s, \bar{\alpha}}$ and take $\Phi_{\bar{\ell}}$ and $\Lambda_{\bar{\ell}}$ as in the proof of Proposition 5.1, for instance. Then $f$ defines an element of $\left(\widetilde{F}_{p, q}^{s, \bar{\alpha}}\right)^{\prime}$ via

$$
\langle f, g\rangle_{*}:=\sum_{\bar{\ell} \in \mathbb{Z}^{d}}\left\langle\Phi_{\bar{\ell}} * f, \overline{\Lambda_{\bar{\ell}} * g}\right\rangle, \quad \text { where } g \in \widetilde{F}_{p, q}^{s, \bar{\alpha}},
$$

as can be seen by the following estimate,

$$
\begin{aligned}
\left|\langle f, g\rangle_{*}\right| & =\left|\sum_{\bar{\ell} \in \mathbb{Z}^{d}}\left\langle\Phi_{\bar{\ell}} * f, \overline{\Lambda_{\bar{\ell}} * g}\right\rangle\right|=\left|\sum_{\bar{\ell} \in \mathbb{Z}^{d}} \int_{\mathbb{R}^{d}}\left(\Phi_{\bar{\ell}} * f\right)(y) \cdot\left(\Lambda_{\bar{\ell}} * g\right)(y) d y\right| \\
& =\left|\int_{\mathbb{R}^{d}} \sum_{\bar{j} \in \mathbb{N}_{0}^{d}}\left(\Phi_{\bar{j}} * f\right)(y) \cdot\left(\Lambda_{\bar{j}} * g\right)(y) d y\right| \\
& \leq \int_{\mathbb{R}^{d}}\left(\sum_{\bar{j} \in \mathbb{N}_{0}^{d}} 2^{-\|\bar{j} / \bar{\alpha}\|_{\infty} s q^{\prime}}\left|\Phi_{\bar{j}} * f(y)\right|^{q^{\prime}}\right)^{1 / q^{\prime}}\left(\sum_{\bar{j} \in \mathbb{N}_{0}^{d}} 2^{\|\bar{j} / \bar{\alpha}\|_{\infty} s q}\left|\Lambda_{\bar{j}} * g(y)\right|^{q}\right)^{1 / q} d y \\
& \leq\left(\int_{\mathbb{R}^{d}}\left(\sum_{\bar{j} \in \mathbb{N}_{0}^{d}} 2^{-\|\bar{j} / \bar{\alpha}\|_{\infty} s q^{\prime}}\left|\Phi_{\bar{j}} * f(y)\right|^{q^{\prime}}\right)^{p^{\prime} / q^{\prime}} d y\right)^{1 / p^{\prime}} \\
& \times\left(\int_{\mathbb{R}^{d}}\left(\sum_{\bar{j} \in \mathbb{N}_{0}^{d}} 2^{\|\bar{j} / \bar{\alpha}\|_{\infty} s q}\left|\Lambda_{\bar{j}} * g(y)\right|^{q}\right)^{p / q} d y\right)^{1 / p} \\
& \lesssim\|f\|_{\widetilde{F}_{p^{\prime}, q^{\prime}}^{-s, \bar{\alpha}} \cdot\|g\|_{\widetilde{F}_{p, q}^{s, \bar{\alpha}}} .}
\end{aligned}
$$

Hereby, we applied Hölder's inequality and used $\Phi_{\bar{\ell}}=\Lambda_{\bar{\ell}}=0$ if $\bar{\ell} \notin \mathbb{N}_{0}^{d}$.

The duality product $\langle\cdot, \cdot\rangle_{*}$ thus yields an embedding $\iota: \widetilde{F}_{p^{\prime}, q^{\prime}}^{-s, \bar{\alpha}} \rightarrow\left(\widetilde{F}_{p, q}^{s, \bar{\alpha}}\right)^{\prime}$. Further, we have natural embeddings $v: \widetilde{F}_{p^{\prime}, q^{\prime}}^{-s, \bar{\alpha}} \hookrightarrow \mathcal{S}^{\prime}\left(\mathbb{R}^{d}\right)$ and $\kappa:\left(\widetilde{F}_{p, q}^{s, \bar{\alpha}}\right)^{\prime} \hookrightarrow \mathcal{S}^{\prime}\left(\mathbb{R}^{d}\right)$ (see (40)). To establish a bridge between $\kappa, \iota$, and $v$, we now consider the special case of a Schwartz function $g=\phi \in \mathcal{S}\left(\mathbb{R}^{d}\right)$ in (41). We obtain

$$
\langle f, \phi\rangle_{*}=\sum_{\bar{\ell} \in \mathbb{Z}^{d}}\left\langle\Phi_{\bar{\ell}} * f, \Lambda_{\bar{\ell}} * \phi\right\rangle_{\mathcal{S}^{\prime} \times \mathcal{S}}=\sum_{\bar{\ell} \in \mathbb{Z}^{d}}\left\langle\Phi_{\bar{\ell}} * \Lambda_{\bar{\ell}} * f, \phi\right\rangle_{\mathcal{S}^{\prime} \times \mathcal{S}}=\langle f, \phi\rangle_{\mathcal{S}^{\prime} \times \mathcal{S}}
$$

Hence, the above (somewhat artificially) defined operation of $f$ on $\widetilde{F}_{p, q}^{s, \bar{\alpha}}$ via $\langle\cdot, \cdot\rangle_{*}$ is compatible with the operation of $f$ as an element of $\mathcal{S}^{\prime}\left(\mathbb{R}^{d}\right)$ on $\mathcal{S}\left(\mathbb{R}^{d}\right)$. This proves $v=\kappa \circ \iota$ and thus $\widetilde{F}_{p^{\prime}, q^{\prime}}^{-s, \bar{\alpha}} \subset\left(\widetilde{F}_{p, q}^{s, \bar{\alpha}}\right)^{\prime}$, considered as subsets of $\mathcal{S}^{\prime}\left(\mathbb{R}^{d}\right)$.

It remains to prove the converse inclusion $\left(\widetilde{F}_{p, q}^{s, \bar{\alpha}}\right)^{\prime} \subset \widetilde{F}_{p^{\prime}, q^{\prime}}^{-s, \bar{\alpha}}$. For this, let $f \in \mathcal{S}^{\prime}\left(\mathbb{R}^{d}\right)$ be an element of $\left(\widetilde{F}_{p, q}^{s, \bar{\alpha}}\right)^{\prime}$. We will show that this implies $f \in \widetilde{F}_{p^{\prime}, q^{\prime}}^{-s, \bar{\alpha}}$ and to this end start with a construction of an isometric embedding

$$
\mu:\left(\widetilde{F}_{p, q}^{s, \bar{\alpha}}\right)^{\prime} \rightarrow L_{p^{\prime}}\left(\mathbb{R}^{d}, \ell_{q^{\prime}}\right), \quad f \mapsto\left(f_{\bar{j}}\right)_{\bar{j}}
$$


Thereby, we build upon the observation that the assignment $g \mapsto\left(2^{\|\bar{j} / \bar{\alpha}\|_{\infty} s} \Delta_{\bar{j}} g\right)_{\bar{j}}$ maps $\widetilde{F}_{p, q}^{s, \bar{\alpha}}$ isometrically to a closed subspace of $L_{p}\left(\mathbb{R}^{d}, \ell_{q}\right)$. Via this assignment and the Hahn-Banach extension theorem, it is therefore possible to identify each functional $f \in\left(\widetilde{F}_{p, q}^{s, \bar{\alpha}}\right)^{\prime}$ with a functional on $L_{p}\left(\mathbb{R}^{d}, \ell_{q}\right)$ having the same norm. Invoking Proposition $5.7(\mathrm{i})$, this then yields an associated family $\left(f_{\bar{j}}\right)_{\bar{j}} \in L_{p^{\prime}}\left(\mathbb{R}^{d}, \ell_{q^{\prime}}\right)$ with $\left\|\left(f_{\bar{j}}\right)_{\bar{j}}\right\|_{L_{p^{\prime}}\left(\mathbb{R}^{d}, \ell_{q^{\prime}}\right)}=\|f\|_{\left(\widetilde{F}_{p, q}^{s, \bar{\alpha})^{\prime}}\right.}$ and $\langle f, g\rangle=\sum_{\bar{j}}\left\langle f_{\bar{j}}, 2^{\|\bar{j} / \bar{\alpha}\|_{\infty} s} \Delta_{\bar{j}} g\right\rangle$, establishing (42).

In particular, for every $\phi \in \mathcal{S}\left(\mathbb{R}^{d}\right)$

$$
\langle f, \phi\rangle=\sum_{\bar{j}}\left\langle f_{\bar{j}}, 2^{\|\bar{j} / \bar{\alpha}\|_{\infty} s} \Delta_{\bar{j}} \phi\right\rangle=\sum_{\bar{j}}\left\langle\Delta_{\bar{j}} \tilde{f}_{\bar{j}}, \phi\right\rangle,
$$

with $\tilde{f}_{\bar{j}}:=2^{\|\bar{j} / \bar{\alpha}\|_{\infty} s} f_{\bar{j}}$. Hence, we have

$$
f=\sum_{\bar{j}} 2^{\|\bar{j} / \bar{\alpha}\|_{\infty} s} \Delta_{\bar{j}} f_{\bar{j}}=\sum_{\bar{j}} \Delta_{\bar{j}} \tilde{f}_{\bar{j}} \quad \text { weak*ly in } \mathcal{S}^{\prime}\left(\mathbb{R}^{d}\right) .
$$

Further, it holds $\left\|\left(2^{-\|\bar{j} / \bar{\alpha}\|_{\infty} s} \tilde{f}_{\bar{j}}\right)_{\bar{j} \in \mathbb{N}_{0}^{d}}\right\|_{\ell_{q^{\prime}}\left(L_{p^{\prime}}\right)}=\left\|\left(f_{\bar{j}}\right)_{\bar{j} \in \mathbb{N}_{0}^{d}}\right\|_{\ell_{q^{\prime}}\left(L_{p^{\prime}}\right)}=\|f\|_{\left(\widetilde{F}_{p}^{s, q}, \bar{\alpha}\right)^{\prime}}$ In view of Proposition 5.8 (i), this shows $f \in \widetilde{F}_{p^{\prime}, q^{\prime}}^{-s, \bar{\alpha}}$ and finishes the proof of $\left(\widetilde{F}_{p, q}^{s, \bar{\alpha}}\right)^{\prime} \cong$ $\widetilde{F}_{p^{\prime}, q^{\prime}}^{-s, \bar{\alpha}}$.

We next establish $\left(\tilde{f}_{p, q}^{s, \bar{\alpha}}\right)^{\prime} \cong \tilde{f}_{p^{\prime}, q^{\prime}}^{-s, \bar{\alpha}}$, which can be elegantly done using the previous result together with the wavelet isomorphism $\lambda: \widetilde{F}_{p, q}^{s, \bar{\alpha}} \rightarrow \tilde{f}_{p, q}^{s, \bar{\alpha}}$ established in Theorem 4.6. For this, we first verify that $\lambda$ preserves the duality structure of $\widetilde{F}_{p^{\prime}, q^{\prime}}^{-s, \bar{\alpha}} \times \widetilde{F}_{p, q}^{s, \bar{\alpha}}$. Indeed, for $f \in \widetilde{F}_{p^{\prime}, q^{\prime}}^{-s, \bar{\alpha}}$ and $g \in \widetilde{F}_{p, q}^{s, \bar{\alpha}}$ we have

$$
\begin{aligned}
\langle f, g\rangle_{\widetilde{F}_{p^{\prime}, q^{\prime}}^{-s, \bar{\alpha}} \times \widetilde{F}_{p, q}^{s, \bar{\alpha}}} & =\left\langle\sum_{\bar{j}, \bar{k}} 2^{\|\bar{j}\|_{1}}\left\langle f, \psi_{\bar{j}, \bar{k}}\right\rangle \psi_{\bar{j}, \bar{k}}, g\right\rangle_{\widetilde{F}_{p^{\prime}, q^{\prime}}^{-s, \bar{\alpha}} \times \widetilde{F}_{p, q}^{s, \bar{\alpha}}} \\
& =\sum_{\bar{j}, \bar{k}} 2^{\|\bar{j}\|_{1}}\left\langle f, \psi_{\bar{j}, \bar{k}}\right\rangle\left\langle\psi_{\bar{j}, \bar{k}}, g\right\rangle_{\widetilde{F}_{p^{\prime}, q^{\prime}}^{-s, \bar{\alpha}} \times \widetilde{F}_{p, q}^{s, \bar{\alpha}}} \\
& =\sum_{\bar{j}, \bar{k}} 2^{-\|\bar{j}\|_{1}}\left(2^{\|\bar{j}\|_{1}}\left\langle f, \psi_{\bar{j}, \bar{k}}\right\rangle\right)\left(2^{\|\bar{j}\|_{1}}\left\langle g, \psi_{\bar{j}, \bar{k}}\right\rangle\right) \\
& =\langle\lambda(f), \lambda(g)\rangle_{\tilde{f}_{p^{\prime}, q^{\prime}}^{-s, \bar{\alpha}} \times \tilde{f}_{p, q}^{s, \bar{\alpha}}} .
\end{aligned}
$$

Note that hereby we relied on the strong convergence of the wavelet expansion in the space $\widetilde{F}_{p^{\prime}, q^{\prime}}^{-s, \bar{\alpha}}$. Next we recall the isomorphism $\iota: \widetilde{F}_{p^{\prime}, q^{\prime}}^{-s, \bar{\alpha}} \rightarrow\left(\widetilde{F}_{p, q}^{s, \bar{\alpha}}\right)^{\prime}$ established above and let $\lambda^{\prime}:\left(\tilde{f}_{p, q}^{s, \bar{\alpha}}\right)^{\prime} \rightarrow\left(\widetilde{F}_{p, q}^{s, \bar{\alpha}}\right)^{\prime}$ denote the dual map of $\lambda$, which is also an isomorphism. 
Then we can read $\left(\tilde{f}_{p, q}^{s, \bar{\alpha}}\right)^{\prime} \cong \tilde{f}_{p^{\prime}, q^{\prime}}^{-s, \bar{\alpha}}$ directly from the following chain of isomorphisms

$$
\left(\tilde{f_{p, q}^{s}}\right)^{s, \bar{\alpha}} \times \tilde{f}_{p, q}^{s, \bar{\alpha}} \stackrel{\lambda^{\prime} \times \lambda^{-1}}{\longrightarrow}\left(\widetilde{F}_{p, q}^{s, \bar{\alpha}}\right)^{\prime} \times \widetilde{F}_{p, q}^{s, \bar{\alpha}} \stackrel{\iota^{-1} \times I d}{\longrightarrow} \widetilde{F}_{p^{\prime}, q^{\prime}}^{-s, \bar{\alpha}} \times \widetilde{F}_{p, q}^{s, \bar{\alpha}} \stackrel{\lambda \times \lambda}{\longrightarrow} \tilde{f}_{p^{\prime}, q^{\prime}}^{-s, \bar{\alpha}} \times \tilde{f}_{p, q}^{s, \bar{\alpha}} .
$$

\section{Hyperbolic and Classical (Anisotropic) Sobolev Spaces}

In the remaining two sections we will analyze the relationship between the newly introduced hyperbolic scale of spaces $\widetilde{A}_{p, q}^{s, \bar{\alpha}}\left(\mathbb{R}^{d}\right)$ from Sect. 3, where $A \in\{B, F\}$, and the classical scale of anisotropic spaces $A_{p, q}^{s, \bar{\alpha}}\left(\mathbb{R}^{d}\right)$, which was recalled in Sect. 2. Our first result shows that, surprisingly, for Sobolev spaces (i.e. the case $A=F$, $1<p<\infty, q=2$ ) both scales coincide.

Theorem 6.1 Let $1<p<\infty, s \in \mathbb{R}$, and $\bar{\alpha}>0$ be an anisotropy vector as above. Then

$$
\widetilde{W}_{p}^{s, \bar{\alpha}}\left(\mathbb{R}^{d}\right)=W_{p}^{s, \bar{\alpha}}\left(\mathbb{R}^{d}\right) \quad \text { (in the sense of equivalent norms). }
$$

Proof The proof is divided into two steps. For convenience, we will thereby abbreviate by $m_{\bar{\alpha}, s}:=\left(\sum_{i=1}^{d}\left(1+\xi_{i}^{2}\right)^{1 /\left(2 \alpha_{i}\right)}\right)^{s}$ the function which appears in the definition (4) of $W_{p}^{s, \bar{\alpha}}$.

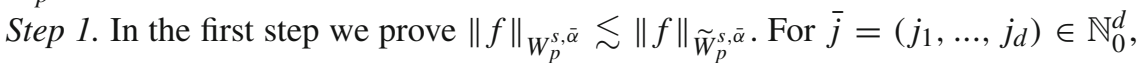
let $\left(\varphi_{j}\right)_{j}$ denote a fixed hyperbolic resolution of unity as introduced in Definition 3.2, with corresponding univariate family $\left(\varphi_{j}\right)_{j}$ where $\operatorname{supp}\left(\varphi_{0}\right) \subset[-2,2]$ and $\varphi_{0} \equiv 1$ on $[-1,1]$, as well as $\varphi_{j}=\varphi_{0}\left(2^{-j} \cdot\right)-\varphi_{0}\left(2^{-(j-1)}\right.$.) for $j \geq 1$. In addition, let us also construct a second hyperbolic resolution of unity $\left(\psi_{\bar{j}}\right)_{\bar{j}}$ such that $\psi_{\bar{j}} \varphi_{\bar{j}}=\varphi_{\bar{j}}$ for every $\bar{j} \in \mathbb{N}_{0}^{d}$. Hereby, it is not possible for $\left(\psi_{j}\right)_{j}$ to obey the same strict building law as formulated in Definition 3.2. We define functions

$$
\begin{aligned}
& \psi_{0}^{*}:=\varphi_{0}+\varphi_{1}, \psi_{1}^{*}:=\varphi_{0}+\varphi_{1}+\varphi_{2}, \psi_{2}^{*}:=\varphi_{0}+\varphi_{1}+\varphi_{2}+\varphi_{3}, \\
& \psi_{j}^{*}:=\sum_{r=-1}^{1} \varphi_{j+r} \quad(j \geq 3)
\end{aligned}
$$

and then put $\psi_{j}:=\psi_{j}^{*} / 3$ for $j \in \mathbb{N}_{0}$. Then clearly $\sum_{j} \psi_{j}=1$ and $\psi_{j} \varphi_{j}=\varphi_{j}$. Finally, we set

$$
\psi_{\bar{j}}:=\psi_{j_{1}} \otimes \cdots \otimes \psi_{j_{d}} \text { to obtain }\left(\psi_{\bar{j}}\right)_{\bar{j}}
$$

By construction, $\psi_{0}=\varphi_{0}\left(2^{-1} \cdot\right) / 3, \psi_{j+1}=\psi_{j}\left(2^{-1} \cdot\right)$ for $j \in \mathbb{N}_{0} \backslash\{2\}$, and $\psi_{3}=\psi_{0}\left(2^{-3} \cdot\right)-\psi_{0}$. Also note that $\operatorname{supp}\left(\psi_{0}\right) \subset[-4,4]$ and $\operatorname{supp}\left(\psi_{3}\right) \subset$ 
$[-32,32] \backslash(-2,2)$. As a consequence, we can record supp $\psi_{j}\left(2^{j} \cdot\right) \subset[-4,4]$ for all $j \in \mathbb{N}_{0}$ and $\psi_{j}\left(2^{j} \cdot\right)=0$ on $[-1 / 4,1 / 4]$ when $j \geq 3$.

Utilizing $\left(\psi_{\bar{j}}\right)_{\bar{j}}$, we then first rewrite the $W_{p}^{s, \bar{\alpha}}$-norm as follows,

$$
\begin{aligned}
& \|f\|_{W_{p}^{s, \bar{\alpha}}} \asymp\left\|\mathcal{F}^{-1}\left[m_{\bar{\alpha}, s} \mathcal{F} f\right]\right\|_{p} \\
& \asymp\left\|\left(\sum_{\bar{j} \in \mathbb{N}_{0}^{d}}\left|\mathcal{F}^{-1} \varphi_{\bar{j}} m_{\bar{\alpha}, s} \mathcal{F} f\right|^{2}\right)^{\frac{1}{2}}\right\|_{p}
\end{aligned}
$$

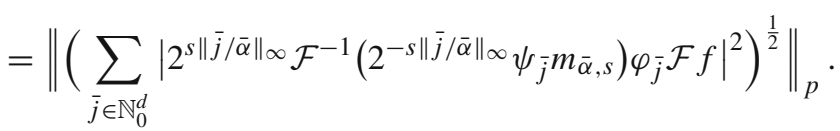

The second equivalence is an anisotropic Littlewood-Paley theorem stating that the concrete space $L^{p}$ and the anisotropic Triebel-Lizorkin space $F_{p, 2}^{0, \bar{\alpha}}$ coincide with equivalent norms (applied to the function $g=\mathcal{F}^{-1}\left[m_{\bar{\alpha}, s} \mathcal{F} f\right]$ ) as mentioned in Sect. 2 (see e.g. [42, Sect. 4.2.2] or [47, Sect. 2.5.2] for a proof).

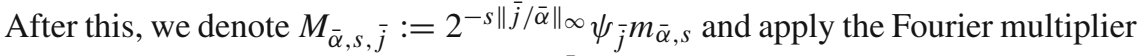

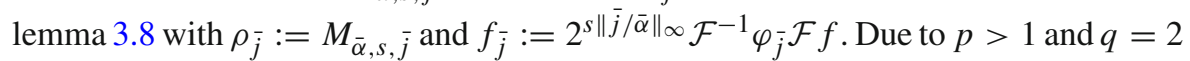
we can thereby choose $r:=2$. This leads to

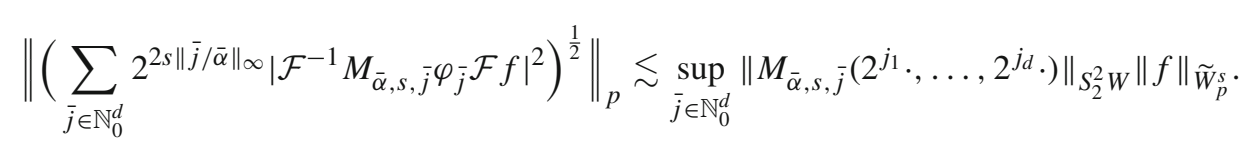

To finish Step 1, it now merely remains to check whether $\sup _{\bar{j} \in \mathbb{N}_{0}^{d}} \| M_{\bar{\alpha}, s, \bar{j}}\left(2^{j_{1}} \cdot \ldots\right.$, $\left.2^{j_{d}} \cdot\right) \|_{S_{2}^{2} W}$ is finite. But, defining $\tilde{\psi}_{0}:=\psi_{0}$ and $\tilde{\psi}_{1}:=\psi_{3}\left(2^{3} \cdot\right)$ with support in

$$
I_{0}:=[-4,4] \text { and } I_{1}:=[-4,4] \backslash(-1 / 4,1 / 4),
$$

respectively, and letting $\tilde{\psi}_{\bar{r}}:=\tilde{\psi}_{r_{1}} \otimes \cdots \otimes \tilde{\psi}_{r_{d}}$ for $\bar{r}=\left(r_{1}, \ldots, r_{d}\right) \in\{0,1\}^{d}$, we have

$$
M_{\bar{\alpha}, s, \bar{j}}\left(2^{j_{1}} \xi_{1}, \ldots, 2^{j_{d}} \xi_{d}\right)=2^{-s\|\bar{j} / \bar{\alpha}\|_{\infty}} \tilde{\psi}_{s(\bar{j})}\left(\xi_{1}, \ldots, \xi_{d}\right) m_{\bar{\alpha}, s}\left(2^{j_{1}} \xi_{1}, \ldots, 2^{j_{d}} \xi_{d}\right)
$$

where $s(\bar{j}):=\left(\operatorname{sgn}\left(j_{1}-2\right), \ldots, \operatorname{sgn}\left(j_{d}-2\right)\right)_{+}$. And for each $\bar{j} \in \mathbb{N}_{0}^{d}$, the function $\tilde{\psi}_{s(\bar{j})}$ belongs to $\mathcal{S}\left(\mathbb{R}^{d}\right)$ and is supported on

$$
\mathbf{I}_{s(\bar{j})}:=I_{s\left(j_{1}\right)} \times \cdots \times I_{s\left(j_{d}\right)}
$$

where $I_{0}, I_{1}$ are given as in $(43)$ and $s\left(j_{\ell}\right):=\left(\operatorname{sgn}\left(j_{\ell}-2\right)\right)_{+}, \ell \in\{1, \ldots, d\}$. Hence, it is sufficient to verify that the mixed derivatives - up to order 2 in each component - of 


$$
\begin{aligned}
& F_{\bar{\alpha}, s, \bar{j}}:\left(\xi_{1}, \ldots, \xi_{d}\right) \mapsto 2^{-s\|\bar{j} / \bar{\alpha}\|_{\infty}} m_{\bar{\alpha}, s}\left(2^{j_{1}} \xi_{1}, \ldots, 2^{j_{d}} \xi_{d}\right) \\
& =\left(\sum_{i=1}^{d} 2^{-\|\bar{j} / \bar{\alpha}\|_{\infty}}\left(1+2^{2 j_{i}} \xi_{i}^{2}\right)^{1 /\left(2 \alpha_{i}\right)}\right)^{s}
\end{aligned}
$$

are uniformly bounded over $\bar{j} \in \mathbb{N}_{0}^{d}$ and $\xi \in \mathbf{I}_{s(\bar{j})}$. For this, we first observe that

$$
\begin{aligned}
F_{\bar{\alpha}, s, \bar{j}}(\xi) & =\left(\sum_{i=1}^{d} 2^{-\|\bar{j} / \bar{\alpha}\|_{\infty}}\left(1+2^{2 j_{i}} \xi_{i}^{2}\right)^{1 /\left(2 \alpha_{i}\right)}\right)^{s} \\
& =\left(\sum_{i=1}^{d}\left(2^{-2 \alpha_{i}\|\bar{j} / \bar{\alpha}\|_{\infty}}+2^{-2 \alpha_{i}\left(\|\bar{j} / \bar{\alpha}\|_{\infty}-j_{i} / \alpha_{i}\right)} \xi_{i}^{2}\right)^{1 /\left(2 \alpha_{i}\right)}\right)^{s},
\end{aligned}
$$

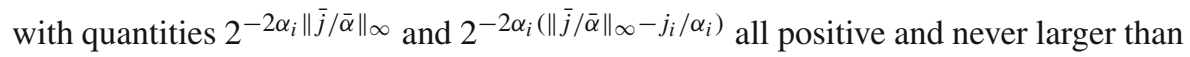
one. This immediately implies

$$
\sup _{\substack{\bar{j} \in \mathbb{N}_{0}^{d} \\ \xi \in[-4,4]^{d}}}\left|F_{\bar{\alpha}, s, \bar{j}}(\xi)\right| \lesssim 1
$$

Next, we determine the partial derivatives $\partial^{\bar{\gamma}} m_{\bar{\alpha}, s}$ of $m_{\bar{\alpha}, s}$ with $\bar{\gamma} \in\{0,1,2\}^{d}$ and $\bar{\gamma} \neq(0, \ldots, 0)$. They are given by

$$
\begin{aligned}
\partial^{\bar{\gamma}} m_{\bar{\alpha}, s}(\xi)= & \sum_{\substack{\bar{\ell}=\left(\ell_{1}, \ldots, \ell_{d}\right) \in \mathbb{N}_{0}^{d} \\
\left(\ell_{1}, \ldots, \ell_{d}\right) \leq\left(\gamma_{1}-1, \ldots, \gamma_{d}-1\right)_{+}}} \frac{s(s-1) \cdots\left(s-\|\bar{\gamma}-\bar{\ell}\|_{1}+1\right)}{\bar{\alpha} \bar{\gamma}-\bar{\ell}} \cdot m_{\bar{\alpha}, s-\|\bar{\gamma}-\bar{\ell}\|_{1}}(\xi) \times \\
& \times \prod_{i=1}^{d}\left[\xi_{i}\left\langle\xi_{i}\right\rangle^{\frac{1}{\alpha_{i}}-2}\right]^{\gamma_{i}-2 \ell_{i}}\left[\left\langle\xi_{i}\right\rangle^{\frac{1}{\alpha_{i}}-4}\left(\left(\frac{1}{\alpha_{i}}-1\right) \xi_{i}^{2}+1\right)\right]^{\ell_{i}},
\end{aligned}
$$

where we use the abbreviation $\left\langle\xi_{\ell}\right\rangle$ for $\left(1+\xi_{\ell}^{2}\right)^{1 / 2}$. We deduce the estimate

$$
\left|\partial^{\bar{\gamma}} m_{\bar{\alpha}, s}(\xi)\right| \lesssim \sum_{\substack{\bar{\ell}=\left(\ell_{1}, \ldots, \ell_{d}\right) \in \mathbb{N}_{d}^{d} \\\left(\ell_{1}, \ldots, \ell_{d}\right) \leq\left(\gamma_{1}-1, \ldots, \gamma_{d}-1\right)_{+}}}\left|m_{\bar{\alpha}, s-\|\bar{\gamma}-\bar{\ell}\|_{1}}(\xi)\right| \cdot \prod_{i=1}^{d}\left[\left\langle\xi_{i}\right\rangle^{\frac{1}{\alpha_{i}}-1}\right]^{\gamma_{i}-2 \ell_{i}}\left[\left\langle\xi_{i}\right\rangle^{\frac{1}{\alpha_{i}}-2}\right]^{\ell_{i}}
$$

and thus, using

$$
\begin{aligned}
& \prod_{i=1}^{d}\left[\left\langle\xi_{i}\right\rangle^{\frac{1}{\alpha_{i}}-1}\right]^{\gamma_{i}-2 \ell_{i}}\left[\left\langle\xi_{i}\right\rangle^{\frac{1}{\alpha_{i}}-2}\right]^{\ell_{i}}=\prod_{i=1}^{d}\left\langle\xi_{i}\right\rangle^{\left(\gamma_{i}-\ell_{i}\right) / \alpha_{i}}\left\langle\xi_{i}\right\rangle^{-\gamma_{i}},
\end{aligned}
$$

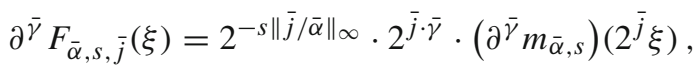

$$
\begin{aligned}
& m_{\bar{\alpha}, s-\|\bar{\gamma}-\bar{\ell}\|_{1}}\left(2^{\bar{j}} \xi\right)=2^{\left(s-\|\bar{\gamma}-\bar{\ell}\|_{1}\right)\|\bar{j} / \bar{\alpha}\|_{\infty}} F_{\bar{\alpha}, s-\|\bar{\gamma}-\bar{\ell}\|_{1}, \bar{j}}(\xi) \text {, }
\end{aligned}
$$


arrive at the inequality

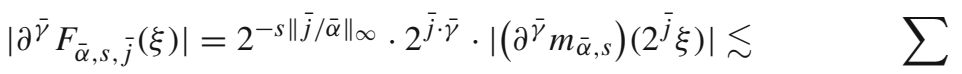

$$
\begin{aligned}
& \bar{\ell}=\left(\ell_{1}, \ldots, \ell_{d}\right) \in \mathbb{N}_{0}^{d} \\
& \left(\ell_{1}, \ldots, \ell_{d}\right) \leq\left(\gamma_{1}-1, \ldots, \gamma_{d}-1\right)_{+}
\end{aligned}
$$

with terms

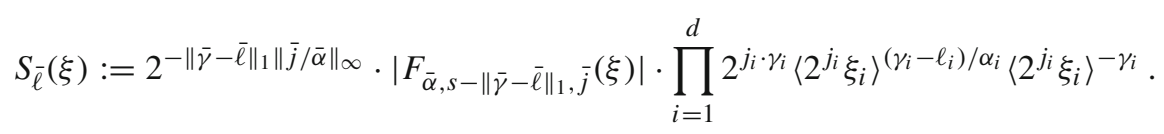

We now use $\left\langle 2^{j_{i}} \xi_{i}\right\rangle \leq 2^{j_{i}}\left\langle\xi_{i}\right\rangle$. Further, if $j_{i} \in\{0,1,2\}$ we estimate $2^{j_{i}}\left\langle 2^{j_{i}} \xi_{i}\right\rangle^{-1} \leq$ $4\left\langle\xi_{i}\right\rangle^{-1} \leq 4$. In case $j_{i} \geq 3$ we consider only $\left|\xi_{i}\right| \geq \frac{1}{4}$ and obtain $2^{j_{i}}\left\langle 2^{j_{i}} \xi_{i}\right\rangle^{-1} \leq$ $\left|\xi_{i}\right|^{-1} \leq 4$. Under the condition $\xi \in \mathbf{I}_{s(\bar{j})}$, we have thus shown

$$
S_{\bar{\ell}}(\xi) \leq 4 \cdot 2^{-\|\bar{\gamma}-\bar{\ell}\|_{1}\|\bar{j} / \bar{\alpha}\|_{\infty}} \cdot\left|F_{\bar{\alpha}, s-\|\bar{\gamma}-\bar{\ell}\|_{1}, \bar{j}}(\xi)\right| \cdot 2^{(\bar{\gamma}-\bar{\ell}) \cdot(\bar{j} / \bar{\alpha})} \cdot \prod_{i=1}^{d}\left\langle\xi_{i}\right\rangle^{\left(\gamma_{i}-\ell_{i}\right) / \alpha_{i}}
$$

Observing $(\bar{\gamma}-\bar{\ell}) \cdot(\bar{j} / \bar{\alpha}) \leq\|\bar{\gamma}-\bar{\ell}\|_{1}\|\bar{j} / \bar{\alpha}\|_{\infty}$ and taking (44) into account, we finally realize that the terms $S_{\bar{\ell}}(\xi)$ are uniformly bounded in the range $\xi \in \mathbf{I}_{s(\bar{j})}$ with respect to $\bar{j} \in \mathbb{N}_{0}^{d}$. Step 1 is finished.

Step 2. For the proof of the converse inequality $\|f\|_{\widetilde{W}_{p}^{s, \bar{\alpha}}} \lesssim\|f\|_{W_{p}^{s, \bar{\alpha}}}$ we argue analogously to Step 1 and use this time the multiplier

$$
\tilde{M}_{\bar{\alpha}, s, \bar{j}}(\xi):=\frac{\psi_{\bar{j}}(\xi) 2^{s\|\bar{j} / \bar{\alpha}\|_{\infty}}}{m_{\bar{\alpha}, s}(\xi)}
$$

It is well-defined since $m_{\bar{\alpha}, s}>0$, and we have, using the same notation as in Step 1,

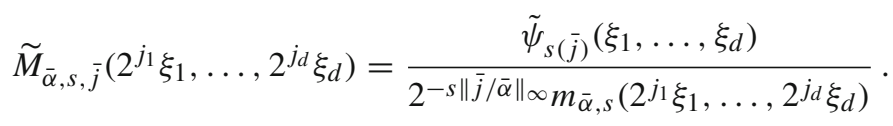

Again, it is not difficult to check that the mixed derivatives of order at most 2 in each component are bounded on $\mathbf{I}_{s(\bar{j})}$ independently of $\bar{j}$. get

By Lemma 3.8, applied with $\rho_{\bar{j}}:=\widetilde{M}_{\bar{\alpha}, s, \bar{j}}, f_{\bar{j}}:=\mathcal{F}^{-1} m_{\bar{\alpha}, s} \varphi_{\bar{j}} \mathcal{F} f$, and $r:=2$, we

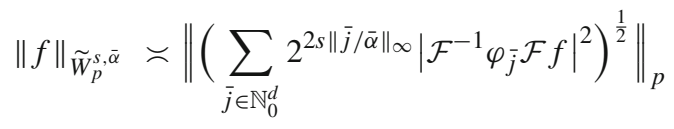

$$
\begin{aligned}
& =\left\|\left(\sum_{\bar{j} \in \mathbb{N}_{0}^{d}} 2^{2 s\|\bar{j} / \bar{\alpha}\|_{\infty}}\left|\mathcal{F}^{-1} \psi_{\bar{j}} m_{\bar{\alpha}, s}^{-1} m_{\bar{\alpha}, s} \varphi_{\bar{j}} \mathcal{F} f\right|^{2}\right)^{\frac{1}{2}}\right\|_{p}
\end{aligned}
$$




$$
\begin{aligned}
& =\left\|\left(\sum_{\bar{j} \in \mathbb{N}_{0}^{d}}\left|\mathcal{F}^{-1} \tilde{M}_{\bar{\alpha}, s, \bar{j}} m_{\bar{\alpha}, s} \varphi_{j} \mathcal{F} f\right|^{2}\right)^{\frac{1}{2}}\right\|_{p} \\
& \lesssim\left(\sup _{\bar{j} \in \mathbb{N}_{0}^{d}}\left\|\tilde{M}_{\bar{\alpha}, s, \bar{j}}\left(2^{j_{1}} \cdot, \ldots, 2^{j_{d}} \cdot\right)\right\|_{S_{2}^{2} W}\right) \cdot\left\|\left(\sum_{\bar{j} \in \mathbb{N}_{0}^{d}}\left|\left(\mathcal{F}^{-1}\left[\varphi_{j} m_{\bar{\alpha}, s} \mathcal{F} f\right]\right)\right|^{2}\right)^{\frac{1}{2}}\right\|_{p} \\
& \lesssim\left\|\mathcal{F}^{-1}\left[m_{\bar{\alpha}, s} \mathcal{F} f\right]\right\|_{p} \asymp\|f\|_{W_{p}^{s, \bar{\alpha}} .}
\end{aligned}
$$

Remark 6.2 We mention that, in contrast to this result, in case $A=B$ we only have coincidence when $p=q=2$. A proof can be found in [1].

As a direct consequence of Theorems 6.1 and 4.6, we obtain new characterizations of classical Sobolev spaces via hyperbolic wavelets.

Theorem 6.3 Let $1<p<\infty, s \in \mathbb{R}$, and $\bar{\alpha}=\left(\alpha_{1}, \ldots, \alpha_{d}\right)>0$ such that $\sum_{i=1}^{d} \alpha_{i}=$ $d$. Let further $\psi_{0}, \psi$ be wavelets satisfying $(K)$ and $(L)$ with

$$
K, L>\sigma_{p, 2}+|s| / \alpha_{\min }
$$

Then any $f \in \mathcal{S}^{\prime}\left(\mathbb{R}^{d}\right)$ belongs to $W_{p}^{s, \bar{\alpha}}\left(\mathbb{R}^{d}\right)$ if and only if it can be represented as

$$
f=\sum_{\bar{j} \in \mathbb{N}_{0}^{d}} \sum_{\bar{k} \in \mathbb{Z}^{d}} \lambda_{\bar{j}, \bar{k}} \psi_{\bar{j}, \bar{k}}
$$

with $\left(\lambda_{\bar{j}, \bar{k}}\right)_{\bar{j}, \bar{k}} \in \tilde{f}_{p, 2}^{s, \bar{\alpha}}$. The representation (45) converges unconditionally in $\mathcal{S}^{\prime}\left(\mathbb{R}^{d}\right)$ and in $W_{p}^{s, \bar{\alpha}}\left(\mathbb{R}^{d}\right)$. In addition, $\left(\psi_{\bar{j}, \bar{k}}\right)_{\bar{j}, \bar{k}}$ is an unconditional basis in $W_{p}^{s, \bar{\alpha}}\left(\mathbb{R}^{d}\right)$. The sequence of coefficients $\lambda:=\lambda(f)=\left(\lambda_{\bar{j}, \bar{k}}\right)_{\bar{j}, \bar{k}}$ is uniquely determined via

$$
\lambda_{\bar{j}, \bar{k}}:=\lambda_{\bar{j}, \bar{k}}(f)=2^{\|j\|_{1}}\left\langle f, \psi_{\bar{j}, \bar{k}}\right\rangle
$$

and we have the wavelet isomorphism (equivalent norm)

$$
\|f\|_{W_{p}^{s, \bar{\alpha}}\left(\mathbb{R}^{d}\right)} \asymp\|\lambda(f)\|_{\tilde{f}_{p, 2}^{s, \bar{\alpha}}}, \quad f \in W_{p}^{s, \bar{\alpha}}\left(\mathbb{R}^{d}\right) .
$$

Analogously, combining Theorem 6.1 with Theorem 5.4, we also derive new characterizations of Sobolev spaces with the hyperbolic Haar system $\mathcal{H}_{d}$ from (25).

Theorem 6.4 Let $1<p<\infty, s \in \mathbb{R}$, and $\bar{\alpha}=\left(\alpha_{1}, \ldots, \alpha_{d}\right)>0$ such that $\sum_{i=1}^{d} \alpha_{i}=$ d. Further, assume

$$
|s| / \alpha_{\min }<\min \left\{\frac{1}{p}, 1-\frac{1}{p}\right\} .
$$


Then the Haar system $\mathcal{H}_{d}=\left(h_{\bar{j}, \bar{k}}\right)_{\bar{j}, \bar{k}}$ from (25) constitutes an unconditional basis of $W_{p}^{s, \bar{\alpha}}\left(\mathbb{R}^{d}\right)$ with associated sequence space $\tilde{f}_{p, 2}^{s, \bar{\alpha}}$. The unique sequence of basis coefficients for $f \in W_{p}^{s, \bar{\alpha}}\left(\mathbb{R}^{d}\right)$ is determined by $\lambda:=\lambda(f)=(\lambda \bar{j}, \bar{k})_{\bar{j}, \bar{k}}$ with

$$
\lambda_{\bar{j}, \bar{k}}:=\lambda_{\bar{j}, \bar{k}}(f)=2^{\|\bar{j}\|_{1}}\left\langle f, h_{\bar{j}, \bar{k}}\right\rangle .
$$

Further, we have the wavelet isomorphism (equivalent norm)

$$
\|f\|_{W_{p}^{s, \bar{\alpha}}\left(\mathbb{R}^{d}\right)} \asymp\|\lambda(f)\|_{f_{p, 2}^{s, \bar{\alpha}}}, \quad f \in W_{p}^{s, \bar{\alpha}}\left(\mathbb{R}^{d}\right) .
$$

In addition, those elements of $\mathcal{S}^{\prime}\left(\mathbb{R}^{d}\right)$ belonging to $W_{p}^{s, \bar{\alpha}}\left(\mathbb{R}^{d}\right)$ are characterized by either of the following two criteria:

(i) $f$ can be represented as a sum

$$
f=\sum_{\bar{j} \in \mathbb{N}_{0}^{d}} \sum_{\bar{k} \in \mathbb{Z}^{d}} \lambda_{\bar{j}, \bar{k}} h_{\bar{j}, \bar{k}} \quad \text { converging }\left(\text { weak }^{*} \text { ly) in } \mathcal{S}^{\prime}\left(\mathbb{R}^{d}\right)\right.
$$

with coefficients $(\lambda \bar{j}, \bar{k})_{\bar{j}, \bar{k}} \in \tilde{f}_{p, 2}^{s, \bar{\alpha}}$ (with respect to some chosen ordering).

(ii) With $\lambda(f)$ being defined as in (46), it holds

$$
\lambda(f)=(\lambda \bar{j}, \bar{k})_{\bar{j}, \bar{k}} \in \tilde{f}_{p, 2}^{s, \bar{\alpha}} .
$$

In both cases, the sequence $(\lambda \bar{j}, \bar{k})_{\bar{j}, \bar{k}}$ is necessarily the sequence of basis coefficients and the representation (47) converges unconditionally to $f$ in $W_{p}^{s, \bar{\alpha}}\left(\mathbb{R}^{d}\right)$.

\section{Hyperbolic and Classical (Anisotropic) BLT Spaces}

The next and final theorem of this paper complements the statement of Theorem 6.1, showing that in general the spaces $\widetilde{A}_{p, q}^{s, \bar{\alpha}}\left(\mathbb{R}^{d}\right)$ and $A_{p, q}^{s, \bar{\alpha}}\left(\mathbb{R}^{d}\right)$, with $A \in\{B, F\}$, do not coincide.

Theorem 7.1 Let $0<p, q \leq \infty, s \in \mathbb{R}$, and $\bar{\alpha}=\left(\alpha_{1}, \ldots, \alpha_{d}\right)>0$ with $\sum_{i=1}^{d} \alpha_{i}=$ d.

(i) If $B_{p, q}^{s, \bar{\alpha}}\left(\mathbb{R}^{d}\right)=\widetilde{B}_{p, q}^{s, \bar{\alpha}}\left(\mathbb{R}^{d}\right)$ then $p=q=2$.

(ii) In the range $0<p<\infty$ : If $F_{p, q}^{s, \bar{\alpha}}\left(\mathbb{R}^{d}\right)=\widetilde{F}_{p, q}^{s, \bar{\alpha}}\left(\mathbb{R}^{d}\right)$ then $q=2$ and $1<p<\infty$.

Remark 7.2 The Besov result (i) follows directly from the very general investigations on embeddings between decomposition spaces conducted in [58]. Even more, the findings there allow to strengthen the statement to more general independent parameters, namely

$B_{p_{1}, q_{1}}^{s_{1}, \bar{\alpha}_{1}}\left(\mathbb{R}^{d}\right)=\widetilde{B}_{p_{2}, q_{2}}^{s_{2}, \bar{\alpha}_{2}}\left(\mathbb{R}^{d}\right) \quad \Leftrightarrow \quad p_{1}=p_{2}=q_{1}=q_{2}=2$ and $\bar{\alpha}_{1}=\bar{\alpha}_{2}$ and $s_{1}=s_{2}$. 
The results of [58], however, are not applicable in the proof of (ii) since TriebelLizorkin spaces do not fit into the decomposition space framework. In the sequel, we will therefore give a proof for the $F$-case (ii) which by slight modifications would also provide a direct way to establish the $B$-case (i).

Before we start, let us remind ourselves that the converse statement of (ii), the coincidence of $F_{p, q}^{s, \bar{\alpha}}$ and $\widetilde{F}_{p, q}^{s, \bar{\alpha}}$ when $1<p<\infty$ and $q=2$, is given by Theorem 6.1. The coincidence of $B_{p, q}^{s, \bar{\alpha}}$ and $\widetilde{B}_{p, q}^{s, \bar{\alpha}}$ when $p=q=2$, the converse of (i), is further observed in Remark 6.2.

Proof of Theorem 7.1 (ii) Step 1: Preparation. Fix an anisotropy vector $\bar{\alpha}=\left(\alpha_{1}, \ldots, \alpha_{d}\right)$ and consider a univariate resolution of unity $\left(\theta_{j}\right)_{j \in \mathbb{N}_{0}}$ of the following form:

The generator $\theta_{0} \in \mathcal{S}(\mathbb{R})$ shall satisfy

$$
\operatorname{supp} \theta_{0} \subset\left[-2^{\alpha_{\min } / 3}, 2^{\alpha_{\min } / 3}\right] \quad \text { and } \quad \theta_{0}=1 \text { on }[-1,1] \text {, }
$$

and the functions $\theta_{j}$ for $j \in \mathbb{N}$ shall be obtained via $\theta_{j}(\cdot):=\theta_{0}\left(2^{-j} \cdot\right)-\theta_{0}\left(2^{-(j-1)} \cdot\right)$.

Using $\left(\theta_{j}\right)_{j \in \mathbb{N}_{0}}$, we can then construct two multivariate resolutions of unity on $\mathbb{R}^{d}$. First, via simple tensorization, we get the hyperbolic resolution $\left(\theta_{\bar{j}}\right)_{\bar{j} \in \mathbb{N}_{0}^{d}}$ with

$$
\theta_{\bar{j}}:=\theta_{j_{1}} \otimes \ldots \otimes \theta_{j_{d}}, \quad \bar{j}=\left(j_{1}, \ldots, j_{d}\right) \in \mathbb{N}_{0}^{d} .
$$

It clearly fulfills all the specifications formulated at the beginning of Sect. 3 .

Second, putting $\varphi_{0}^{\bar{\alpha}}:=\theta_{0} \otimes \ldots \otimes \theta_{0}$ and

$$
\varphi_{j}^{\bar{\alpha}}:=\varphi_{0}^{\bar{\alpha}}\left(2^{-j \bar{\alpha}} \cdot\right)-\varphi_{0}^{\bar{\alpha}}\left(2^{-(j-1) \bar{\alpha}} \cdot\right) \text { for } j \in \mathbb{N},
$$

we obtain $\left(\varphi_{j}^{\bar{\alpha}}\right)_{j \in \mathbb{N}_{0}}$, which is a classical anisotropic resolution of unity in compliance with the definition from Sect. 2.1.

For parameters $\alpha>0$ and $\ell \in \mathbb{N}$, let us next introduce the intervals

$$
I_{\ell}^{\alpha}:=2^{(\ell-1) \alpha} \cdot\left[2^{\alpha_{\min } / 3}, 2^{\alpha}\right] \text { and } J_{\ell}^{\alpha}:=\left[-2^{\ell \alpha}, 2^{\ell \alpha}\right]
$$

Then $\theta_{0}\left(2^{-\ell \alpha} \cdot\right)=1$ on $J_{\ell}^{\alpha}$ and $\theta_{0}\left(2^{-\ell \alpha} \cdot\right)-\theta_{0}\left(2^{-(\ell-1) \alpha} \cdot\right)=1$ on $I_{\ell}^{\alpha}$. In particular, $\theta_{j}=1$ on $I_{j}^{1}$ for every $j \in \mathbb{N}$ and thus $\theta_{\bar{j}}=1$ on $I_{j_{1}}^{1} \times \cdots \times I_{j_{d}}^{1}$. Further, we have $\varphi_{0}^{\bar{\alpha}}\left(2^{-j \bar{\alpha}} \cdot\right)=1$ on $J_{j}^{\alpha_{1}} \times \cdots \times J_{j}^{\alpha_{d}}$ and as a consequence $\varphi_{j}^{\bar{\alpha}}=1$ on $\left(J_{j}^{\alpha_{1}} \times \cdots \times\right.$ $\left.J_{j}^{\alpha_{d}}\right) \backslash\left(J_{j-1}^{\alpha_{1}} \times \cdots \times J_{j-1}^{\alpha_{d}}\right)$. This, in turn, implies $\varphi_{j}^{\bar{\alpha}}=1$ on the subset $I_{j}^{\alpha_{1}} \times \cdots \times$ $I_{j}^{\alpha_{d-1}} \times J_{j}^{\alpha_{d}}$.

Observe now that for every $\ell \in \mathbb{N}$ and every $i \in\{1, \ldots, d\}$ either $I_{\ell}^{\alpha_{i}} \cap I_{\left\lfloor\ell \alpha_{i}\right\rfloor}^{1}$ or $I_{\ell}^{\alpha_{i}} \cap I_{\left\lfloor\ell \alpha_{i}\right\rfloor+1}^{1}$ is a nonempty interval of nonzero length. This is due to the fact that always

$$
2^{\gamma} \cdot \mathcal{L}\left(I_{\ell}^{\alpha_{i}}\right) \leq \mathcal{R}\left(I_{\left\lfloor\ell \alpha_{i}\right\rfloor}^{1}\right) \leq \mathcal{R}\left(I_{\ell}^{\alpha_{i}}\right) \quad \text { or } \quad 2^{\gamma} \cdot \mathcal{L}\left(I_{\left\lfloor\ell \alpha_{i}\right\rfloor+1}^{1}\right) \leq \mathcal{R}\left(I_{\ell}^{\alpha_{i}}\right) \leq \mathcal{R}\left(I_{\left\lfloor\ell \alpha_{i}\right\rfloor+1}^{1}\right),
$$


where $\mathcal{L}(I)$ and $\mathcal{R}(I)$ denote the left resp. right endpoint of a given interval $I=[a, b]$ and $\gamma=\alpha_{\min }^{2} / 8$. The verification of this fact is postponed to Step 3 at the end of this proof.

As a consequence, for each $i \in\{1, \ldots, d\}$ and each $\ell \in \mathbb{N}$, we may pick one of those intersections with nonvanishing interior and denote it by $\tilde{I}_{\ell}^{(i)}$. Depending on our choice, we then either have

$$
\tilde{I}_{\ell}^{(i)}=I_{\ell}^{\alpha_{i}} \cap I_{\left\lfloor\ell \alpha_{i}\right\rfloor}^{1} \text { or } \quad \tilde{I}_{\ell}^{(i)}=I_{\ell}^{\alpha_{i}} \cap I_{\left\lfloor\ell \alpha_{i}\right\rfloor+1}^{1} .
$$

Due to the nonvanishing interior of $\tilde{I}_{\ell}^{(i)}$ we can further fix nontrivial functions

$$
h_{\ell}^{[i]} \in \mathcal{S}(\mathbb{R}) \quad \text { with } \operatorname{supp} h_{\ell}^{[i]} \subset \tilde{I}_{\ell}^{(i))}, \quad i \in\{1, \ldots, d-1\} .
$$

With this preparation we are finally ready for the main argumentation.

Step 2: Main Proof. For $\ell \in \mathbb{N}$ let us consider $g_{\ell}: \mathbb{R} \rightarrow \mathbb{C}$ with the property

$$
\operatorname{supp}\left(\mathcal{F} g_{\ell}\right) \subset J_{\ell}^{\alpha_{d}}=\left[-2^{\ell \alpha_{d}}, 2^{\ell \alpha_{d}}\right]
$$

and associate a multivariate function $f_{\ell}: \mathbb{R}^{d} \rightarrow \mathbb{C}$ defined by its Fourier transform

$$
\mathcal{F} f_{\ell}\left(\xi_{1}, \ldots, \xi_{d}\right):=h_{\ell}^{[1]}\left(\xi_{1}\right) h_{\ell}^{[2]}\left(\xi_{2}\right) \cdots h_{\ell}^{[d-1]}\left(\xi_{d-1}\right) \mathcal{F} g_{\ell}\left(\xi_{d}\right)
$$

where $h_{\ell}^{[i]}$ are the functions introduced at the end of Step 1.

Since $\mathcal{F} f_{\ell}$ is supported inside $I_{\ell}^{\alpha_{1}} \times \cdots \times I_{\ell}^{\alpha_{d-1}} \times J_{\ell}^{\alpha_{d}}$, on which $\varphi_{\ell}^{\bar{\alpha}}=1$ according to our considerations in Step 1, we can easily compute the classical anisotropic TriebelLizorkin (quasi-)norm of $f_{\ell}$. Denoting by $\left(\Delta_{j}^{\varphi}\right)_{j \in \mathbb{N}_{0}}$ the Littlewood-Paley analysis associated to $\left(\varphi_{j}^{\bar{\alpha}}\right)_{j \in \mathbb{N}_{0}}$, we have

$$
\left\|f_{\ell}\right\|_{F_{p, q}^{s, \bar{\alpha}}\left(\mathbb{R}^{d}\right)}=\left\|\left(\sum_{j \geq 0} 2^{j s q}\left|\Delta_{j}^{\varphi} f_{\ell}\right|^{q}\right)^{\frac{1}{q}}\right\|_{p}=2^{\ell s}\left\|\Delta_{\ell}^{\varphi} f_{\ell}\right\|_{p}=2^{\ell s}\left\|f_{\ell}\right\|_{p} .
$$

Moreover, as $f_{\ell}$ is a tensor product, we can compute

$$
\left\|f_{\ell}\right\|_{p}=\left\|\mathcal{F}^{-1}\left(h_{\ell}^{[1]}\right)\right\|_{p} \cdot \ldots \cdot\left\|\mathcal{F}^{-1}\left(h_{\ell}^{[d-1]}\right)\right\|_{p}\left\|g_{\ell}\right\|_{p}=C_{\ell}\left\|g_{\ell}\right\|_{p}
$$

with $C_{\ell}:=C_{\ell}^{(1)} \cdot \ldots \cdot C_{\ell}^{(d-1)}$ and $C_{\ell}^{(i)}:=\left\|\mathcal{F}^{-1}\left(h_{\ell}^{[i]}\right)\right\|_{p}$ for $i \in\{1, \ldots, d-1\}$. Altogether, we end up with

$$
\left\|f_{\ell}\right\|_{F_{p, q}^{s, \bar{\alpha}}} \asymp 2^{\ell s} C_{\ell}\left\|g_{\ell}\right\|_{p} .
$$

We proceed with the computation of the hyperbolic Triebel-Lizorkin (quasi-)norm of $f_{\ell}$. It follows right from the definition of the intervals $\tilde{I}_{\ell}^{(i)}$ from (49) that there exist numbers $k_{i}(\ell) \in \mathbb{N}$, either taking the value $\left\lfloor\ell \alpha_{i}\right\rfloor$ or the value $\left\lfloor\ell \alpha_{i}\right\rfloor+1$, such that 
$\tilde{I}_{\ell}^{(i)} \subset I_{k_{i}(\ell)}^{1}$. Hence, due to $\operatorname{supp} \mathcal{F} f_{\ell} \subset \tilde{I}_{\ell}^{(1)} \times \cdots \times \tilde{I}_{\ell}^{(d-1)} \times J_{\ell}^{\alpha_{d}}$ the function $\mathcal{F} f_{\ell}$ is supported inside $I_{k_{1}(\ell)}^{1} \times \cdots \times I_{k_{d-1}(\ell)}^{1} \times J_{\ell}^{\alpha_{d}}$.

Let now $\left(\Delta_{\bar{j}}^{\theta}\right)_{\bar{j} \in \mathbb{N}_{0}^{d}}$ denote the Littlewood-Paley analysis corresponding to $\left(\theta_{\bar{j}}\right)_{\bar{j} \in \mathbb{N}_{0}^{d}}$ and let us abbreviate $k_{i}(\ell)$ by $k_{i}$ and the vector $\left(k_{1}, \ldots, k_{d-1}, j_{d}\right)$ by $\bar{\ell}_{j_{d}}$. Then, since

$$
\sum_{j_{d}=0}^{\left\lfloor\ell \alpha_{d}\right\rfloor+1} \theta_{\bar{\ell}_{j_{d}}}=1 \text { on } I_{k_{1}}^{1} \times \cdots \times I_{k_{d-1}}^{1} \times J_{\ell}^{\alpha_{d}}
$$

we calculate for the hyperbolic Triebel-Lizorkin (quasi-)norm of $f_{\ell}$

$$
\begin{aligned}
\left\|f_{\ell}\right\|_{\tilde{F}_{p, q}^{s, \bar{\alpha}}} & =\left\|\left(\sum_{\bar{j} \in \mathbb{N}_{0}^{d}} 2^{\max \left\{j_{1} / \alpha_{1}, \ldots, j_{d} / \alpha_{d}\right\} s q}\left|\Delta_{\bar{j}}^{\theta} f_{\ell}(\cdot)\right|^{q}\right)^{1 / q}\right\|_{p} \\
& =\left\|\left(\sum_{j_{d}=0}^{\left\lfloor\ell \alpha_{d}\right\rfloor+1} 2^{\max \left\{k_{1} / \alpha_{1}, \ldots, k_{d-1} / \alpha_{d-1}, j_{d} / \alpha_{d}\right\} s q}\left|\Delta_{\bar{\ell}_{j_{d}}}^{\theta} f_{\ell}(\cdot)\right|^{q}\right)^{1 / q}\right\|_{p} \\
& \asymp 2^{\ell s}\left\|\left(\sum_{j_{d}=0}^{\left\lfloor\ell \alpha_{d}\right\rfloor+1}\left|\Delta_{\bar{\ell}_{j_{d}}}^{\theta} f_{\ell}(\cdot)\right|^{q}\right)^{1 / q}\right\|_{p}=2^{\ell s} C_{\ell}\left\|g_{\ell}\right\|_{F_{p, q}^{0}}
\end{aligned}
$$

with the same constant $C_{\ell}$ as obtained before in the computation of $\left\|f_{\ell}\right\|_{F_{p, q}^{s, \bar{\alpha}}}$.

Now we come to the core argument. Assuming that the spaces $F_{p, q}^{s, \alpha, q}\left(\mathbb{R}^{d}\right)$ and $\widetilde{F}_{p, q}^{s, \bar{\alpha}}\left(\mathbb{R}^{d}\right)$ coincide, the associated (quasi-)norms would be equivalent. By our calculations, this would imply that $\left\|g_{\ell}\right\|_{F_{p, q}^{0}(\mathbb{R})}$ is equivalent to $\left\|g_{\ell}\right\|_{L_{p}(\mathbb{R})}$ for any band-limited function $g_{\ell}$ with frequency support as in (50). Moreover, since the proof holds true for all $\ell \in \mathbb{N}$ this equivalence remains valid for any band-limited function $g$ on $\mathbb{R}$.

But, as a consequence of Lemma 7.3(iii), since the sequence $\left(f_{N}^{(3)}\right)_{N}$ constructed in its proof consists of band-limited functions, this is only possible in the range $1<$ $p<\infty$. Furthermore, if $1<p<\infty$ the band-limited functions are dense in $L_{p}(\mathbb{R})$ as well as $F_{p, q}^{0}(\mathbb{R})$. Hence, by Lemma 7.3(i) also $q=2$ is a necessary condition. It now only remains to verify (48).

Step 3: Proof of (48). We distinguish two cases depending on the size of the quantity $\delta:=\ell \alpha_{i}-\left\lfloor\ell \alpha_{i}\right\rfloor \in[0,1)$. Let us subsequently abbreviate $\rho:=\frac{\alpha_{\min }}{4+\alpha_{\min }}$ and $\sigma:=$ $\frac{8 \alpha_{\min }}{3 \alpha_{\min }+12}=\frac{2}{3}(1-\rho) \alpha_{\min }$. Recalling that $\alpha_{\min }=\min _{i \in\{1, \ldots, d\}}\left\{\alpha_{i}\right\} \in(0,1]$, we note that $\rho \in\left(0, \frac{1}{5}\right]$ and $\sigma \in\left(0, \frac{2}{3}\right]$.

In case $\delta \in[0, \sigma)$ we have $\delta \leq(1-\rho)\left(\alpha_{i}-\alpha_{\min } / 3\right)$ and thus

$$
\begin{aligned}
\log _{2} \mathcal{R}\left(I_{\left\lfloor\ell \alpha_{i}\right\rfloor}^{1}\right) & =\left\lfloor\ell \alpha_{i}\right\rfloor=\ell \alpha_{i}-\delta \geq \ell \alpha_{i}-(1-\rho)\left(\alpha_{i}-\alpha_{\min } / 3\right) \\
& =(\ell-1) \alpha_{i}+\alpha_{\min } / 3+\rho\left(\alpha_{i}-\alpha_{\min } / 3\right) \geq \log _{2} \mathcal{L}\left(I_{\ell}^{\alpha_{i}}\right)+\frac{2}{3} \rho \alpha_{\min },
\end{aligned}
$$


where we used $\mathcal{L}\left(I_{\ell}^{\alpha_{i}}\right)=2^{(\ell-1) \alpha_{i}+\alpha_{\min } / 3}$ and $\mathcal{R}\left(I_{\left\lfloor\ell \alpha_{i}\right\rfloor}^{1}\right)=2^{\left\lfloor\ell \alpha_{i}\right\rfloor}$. In view of $\gamma=$ $\alpha_{\min }^{2} / 8<\frac{2}{3} \rho \alpha_{\min }$ and since we always have $\left\lfloor\ell \alpha_{i}\right\rfloor \leq \ell \alpha_{i} \leq\left\lfloor\ell \alpha_{i}\right\rfloor+1$, i.e.

$$
\log _{2} \mathcal{R}\left(I_{\left\lfloor\ell \alpha_{i}\right\rfloor}^{1}\right) \leq \log _{2} \mathcal{R}\left(I_{\ell}^{\alpha_{i}}\right) \leq \log _{2} \mathcal{R}\left(I_{\left\lfloor\ell \alpha_{i}\right\rfloor+1}^{1}\right)
$$

the left inequality in (48) is hence valid in the respective range of $\delta$.

In case $\delta \in[\sigma, 1)$ we first estimate

$$
\delta>\frac{7 \alpha_{\min }}{3 \alpha_{\min }+1}=(1-\rho)+\rho \alpha_{\min } / 3 \geq(1-\rho) \alpha_{\min }+\rho \alpha_{\min } / 3
$$

from which we deduce, using $\ell \alpha_{i}=\left\lfloor\ell \alpha_{i}\right\rfloor+\delta$ and $\mathcal{L}\left(I_{\left\lfloor\ell \alpha_{i}\right\rfloor+1}^{1}\right)=2^{\left\lfloor\ell \alpha_{i}\right\rfloor+\alpha_{\min } / 3}$,

$$
\begin{aligned}
\log _{2} \mathcal{R}\left(I_{\ell}^{\alpha_{i}}\right)= & \ell \alpha_{i}>\left\lfloor\ell \alpha_{i}\right\rfloor+(1-\rho) \alpha_{\min }+\rho \alpha_{\min } / 3=\log _{2} \mathcal{L}\left(I_{\left\lfloor\ell \alpha_{i}\right\rfloor+1}^{1}\right) \\
& +\frac{2}{3}(1-\rho) \alpha_{\min } .
\end{aligned}
$$

This time, again taking into account (51) and $\gamma=\alpha_{\min }^{2} / 8<\frac{2}{3}(1-\rho) \alpha_{\min }$, the right inequality in (48) holds true. Altogether, the proof of (48) is thus finished.

The behavior of the $L_{p}$-(quasi-)norms in relation to the $A_{p, q}^{0}$-(quasi-)norms is crucial for the proof of Theorem 7.1. Concretely, we have shown for $A \in\{B, F\}$

$$
A_{p, q}^{s, \bar{\alpha}}\left(\mathbb{R}^{d}\right)=\widetilde{A}_{p, q}^{s, \bar{\alpha}}\left(\mathbb{R}^{d}\right) \Longleftrightarrow\|f\|_{A_{p, q}^{0}(\mathbb{R})} \asymp\|f\|_{L_{p}(\mathbb{R})} \text { for band-limited functions } f \text {. }
$$

On the right-hand side, the (quasi-)norms are thereby all classical and only the univariate case matters. Using known embedding theorems, the exact parameters for equality could therefore be determined (see [49] Sect. 2.3.2 or [44] Theorem 3.1.1., for example).

Prefering a direct and shorter route, the following lemma provides a simple and quantitative argument for what we need. It investigates the behavior of the respective (quasi-)norms for certain sequences of test functions. As a consequence of statement (i), we extract the necessity $p=q=2$ for equality in (52). From (ii) we further obtain $p=q$ in the B-case. Statement (iii) yields $1<p<\infty$ in the F-case. Altogether, this shows that the Sobolev spaces in Theorem 6.1 are precisely those, where equality holds true.

Lemma 7.3 Assume $0<p<\infty, 0<q \leq \infty, A \in\{B, F\}$. There are sequences $\left(f_{N}^{(i)}\right)_{N \in \mathbb{N}}, i \in\left\{1,1^{\prime}, 2,3\right\}$, of functions on $\mathbb{R}$ such that

(i) $\left\|f_{N}^{(1)}\right\|_{p} \gtrsim N^{1 / 2}$ and $\left\|f_{N}^{(1)}\right\|_{A_{p, q}^{0}(\mathbb{R})} \asymp N^{1 / q}$, (i') $\left\|f_{N}^{\left(1^{\prime}\right)}\right\|_{p} \lesssim N^{1 / 2}$ and $\left\|f_{N}^{\left(1^{\prime}\right)}\right\|_{A_{p, q}^{0}(\mathbb{R})} \asymp N^{1 / q}$, 
(ii) $\left\|f_{N}^{(2)}\right\|_{p} \asymp N^{1 / p}$ and $\left\|f_{N}^{(2)}\right\|_{B_{p, q}^{0}(\mathbb{R})} \asymp N^{1 / q}$,

(iii) $\left\|f_{N}^{(3)}\right\|_{p} \asymp 2^{N(1-1 / p)}$ and $\left\|f_{N}^{(3)}\right\|_{F_{p, q}^{0}(\mathbb{R})} \gtrsim \begin{cases}1 & , p<1 \\ N^{1 / p} & , p \geq 1 .\end{cases}$

Remark 7.4 In case $q=\infty$ we need to interpret $N^{1 / q} \asymp 1$. Further, the case $A=B$ with $p=\infty$ is not considered in Lemma 7.3. By an analogous argument, one can show however that (ii) holds true also for $p=\infty$. So, for $B_{\infty, q}^{0}(\mathbb{R})$ we have the necessary condition $q=\infty$ to be equivalent to $L_{\infty}(\mathbb{R})$. It is further not difficult to show that the sequence $\left(f_{N}^{(1)}\right)_{N \in \mathbb{N}_{0}}$ from (i) fulfills $\left\|f_{N}^{(1)}\right\|_{\infty} \nearrow \infty$ whereas $\left\|f_{N}^{(1)}\right\|_{B_{\infty, \infty}^{0}(\mathbb{R})} \asymp 1$. Hence, $B_{\infty, \infty}^{0}(\mathbb{R}) \neq L_{\infty}(\mathbb{R})$.

Proof ad (i), (i'): We provide the proof for $q<\infty$. Let $\bar{\varepsilon}=\left(\varepsilon_{0}, \varepsilon_{1}, \ldots\right) \in\{-1,1\}^{\mathbb{N}_{0}}$ and define

$$
f_{N, \bar{\varepsilon}}:=\sum_{j=0}^{N} \varepsilon_{j} \sum_{k=0}^{2^{j}} \psi_{j, k}
$$

where $\left(\psi_{j, k}\right)_{j, k}$ shall be a compactly supported, orthogonal, and $L_{\infty}$-normalized wavelet system with sufficient vanishing moments and smoothness to characterize the space $A_{p, q}^{0}(\mathbb{R})$. Further, for each $j \in \mathbb{N}_{0}$ and $k \in\left\{0, \ldots, 2^{j}\right\}$, we assume the support condition supp $\left(\psi_{j, k}\right) \subset[0,1]$.

Now we note that in a univariate setting, as considered here, we have the coincidence $\tilde{A}_{p, q}^{0}(\mathbb{R})=A_{p, q}^{0}(\mathbb{R})$. Hence, using the wavelet isomorphism established by Theorem 4.6 for the F-scale and taking into account Remark 4.5 for the B-scale, we immediately obtain

$$
\begin{aligned}
& \left\|f_{N, \bar{\varepsilon}}\right\|_{B_{p, q}^{0}(\mathbb{R})} \asymp\left(\sum_{j=0}^{N}\left\|\sum_{k=0}^{2^{j}} \chi_{j, k}\right\|_{p}^{q}\right)^{1 / q} \asymp N^{1 / q}, \\
& \left\|f_{N, \bar{\varepsilon}}\right\|_{F_{p, q}^{0}(\mathbb{R})} \asymp\left\|\left(\sum_{j=0}^{N}\left|\sum_{k=0}^{2^{j}} \chi_{j, k}\right|^{q}\right)^{1 / q}\right\|_{p} \asymp N^{1 / q},
\end{aligned}
$$

whereby the (quasi-)norms on the left-hand side do not depend on the choice of $\bar{\varepsilon}$.

From here we proceed with a probabilistic argument and interpret $\bar{\varepsilon}$ as a Rademacher random variable. Then, for the expectation of the $L_{p}$-(quasi-)norms over $\bar{\varepsilon}$,

$$
\mathbb{E}_{\bar{\varepsilon}}\left(\left\|f_{N, \bar{\varepsilon}}\right\|_{p}^{p}\right)=\int_{0}^{1} \int_{\mathbb{R}}\left|\sum_{j=0}^{N} r_{j}(t) \sum_{k=0}^{2^{j}} \psi_{j, k}(x)\right|^{p} d x d t,
$$

where $r_{j}(t):=\operatorname{sgn}\left(\sin 2^{j} \pi t\right)$ is the $j$-th Rademacher function. Applying Khintchine's inequality, we obtain from this 


$$
\begin{aligned}
\mathbb{E}_{\bar{\varepsilon}}\left(\left\|f_{N, \bar{\varepsilon}}\right\|_{p}^{p}\right) & =\int_{0}^{1} \int_{0}^{1}\left|\sum_{j=0}^{N} r_{j}(t) \sum_{k=0}^{2^{j}} \psi_{j, k}(x)\right|^{p} d t d x \\
& \asymp \int_{0}^{1}\left(\sum_{j=0}^{N}\left|\sum_{k=0}^{2^{j}} \psi_{j, k}(x)\right|^{2}\right)^{p / 2} d x .
\end{aligned}
$$

Next, we define for $j \in \mathbb{N}_{0}$ the auxiliary functions

$$
F_{j}:=\frac{1}{M}\left|\sum_{k=0}^{2^{j}} \psi_{j, k}(x)\right|^{2} \text { with } M>0 \text { such that }\left\|F_{j}\right\|_{\infty} \leq 1
$$

Observe that $0 \leq F_{j} \leq 1$. Then, in case $0<p \leq 2$,

$$
N^{-p / 2} \cdot \mathbb{E}_{\bar{\varepsilon}}\left(\left\|f_{N, \bar{\varepsilon}}\right\|_{p}^{p}\right) \asymp \int_{0}^{1}\left(\frac{1}{N} \sum_{j=0}^{N} F_{j}(x)\right)^{p / 2} d x \geq \frac{1}{N} \int_{0}^{1} \sum_{j=0}^{N} F_{j}(x) d x,
$$

and $\int_{0}^{1} F_{j}(x) d x \asymp 1$. Hence $\mathbb{E}_{\bar{\varepsilon}}\left(\left\|f_{N, \bar{\varepsilon}}\right\|_{p}^{p}\right) \gtrsim N^{p / 2}$. Also, since $2 / p \geq 1$, with Hölder

$$
\mathbb{E}_{\bar{\varepsilon}}\left(\left\|f_{N, \bar{\varepsilon}}\right\|_{p}^{p}\right) \asymp \int_{0}^{1}\left(\sum_{j=0}^{N} F_{j}(x)\right)^{p / 2} d x \lesssim\left(\int_{0}^{1} \sum_{j=0}^{N} F_{j}(x) d x\right)^{p / 2} \asymp N^{p / 2} .
$$

In case $2<p<\infty$, we again argue with Hölder

$$
\mathbb{E}_{\bar{\varepsilon}}\left(\left\|f_{N, \bar{\varepsilon}}\right\|_{p}^{p}\right) \asymp \int_{0}^{1}\left(\sum_{j=0}^{N} F_{j}(x)\right)^{p / 2} d x \gtrsim\left(\int_{0}^{1} \sum_{j=0}^{N} F_{j}(x) d x\right)^{p / 2} \asymp N^{p / 2} .
$$

Further, since $2 / p<1$,

$$
N^{-p / 2} \cdot \mathbb{E}_{\bar{\varepsilon}}\left(\left\|f_{N, \bar{\varepsilon}}\right\|_{p}^{p}\right) \asymp \int_{0}^{1}\left(\frac{1}{N} \sum_{j=0}^{N} F_{j}(x)\right)^{p / 2} d x \lesssim \frac{1}{N} \int_{0}^{1} \sum_{j=0}^{N} F_{j}(x) d x \asymp 1 .
$$

Altogether, these estimates show $\mathbb{E}_{\bar{\varepsilon}}\left(\left\|f_{N, \bar{\varepsilon}}\right\|_{p}^{p}\right) \asymp N^{p / 2}$. As a consequence, we can choose $f_{N}:=f_{N, \bar{\varepsilon}(N)}$ such that $\left\|f_{N}\right\|_{p}^{p} \gtrsim N^{p / 2}$, or equivalently $\left\|f_{N}\right\|_{p} \gtrsim \sqrt{N}$, proving (i). Analogously, choosing $f_{N}:=f_{N, \bar{\varepsilon}(N)}$ with $\left\|f_{N}\right\|_{p}^{p} \lesssim N^{p / 2}$ proves (i').

ad (ii): With the same wavelet system $\left(\psi_{j, k}\right)_{j, k}$ as before, $L_{\infty}$-normalized, define

$$
f_{N}:=\sum_{j=0}^{N} 2^{j / p} \psi_{j, k(j)},
$$


where $k(j)$ is chosen such that the (spatial) support of the wavelets is mutually disjoint. Then, using again the wavelet isomorphism from Theorem 4.6 and Remark 4.5, we deduce

$$
\begin{aligned}
\left\|f_{N}\right\|_{B_{p, q}^{0}(\mathbb{R})} & =\left\|f_{N}\right\|_{\widetilde{B}_{p, q}^{0}(\mathbb{R})} \asymp\left(\sum_{j=0}^{N}\left\|2^{j / p} \chi_{j, k(j)}\right\|_{p}^{q}\right)^{1 / q} \\
& =\left(\sum_{j=0}^{N}\left(2^{j / p} 2^{-j / p}\right)^{q}\right)^{1 / q} \asymp N^{1 / q} .
\end{aligned}
$$

For the $L_{p}$-(quasi-)norm we obtain, due to the disjoint support,

$$
\left\|f_{N}\right\|_{L_{p}(\mathbb{R})}=\left\|\sum_{j=0}^{N} 2^{j / p} \psi_{j, k(j)}\right\|_{p}=\left(\sum_{j=0}^{N} 2^{j}\left\|\psi_{j, k(j)}\right\|_{p}^{p}\right)^{1 / p} \asymp N^{1 / p} .
$$

ad (iii): Finally, let $\left(\varphi_{j}\right)_{j}$ be a (standard) dyadic resolution of unity, with $\varphi_{0}=1$ in a neighborhood of 0 and $\varphi_{1}=\varphi_{0}(\cdot / 2)-\varphi_{0}$, and put

$$
f_{N}:=\mathcal{F}^{-1} \varphi_{0}\left(2^{-N} \cdot\right) \text {. }
$$

Then $f_{N}=2^{N} F_{1}\left(2^{N} \cdot\right)$. For the $L_{p}$-(quasi-)norm we thus compute

$$
\left\|f_{N}\right\|_{L_{p}(\mathbb{R})}=2^{N}\left\|F_{1}\left(2^{N} \cdot\right)\right\|_{p} \asymp 2^{N(1-1 / p)} .
$$

Turning to the $F_{p, q}^{0}(\mathbb{R})$-(quasi-)norm, for $N \geq 2$, we calculate, writing $\Phi_{0}:=\mathcal{F}^{-1} \varphi_{0}$ and $\Phi_{1}:=\mathcal{F}^{-1} \varphi_{1}$,

$$
\begin{aligned}
\left\|f_{N}\right\|_{F_{p, q}^{0}(\mathbb{R})} & =\left\|\left(\sum_{j=0}^{\infty}\left|\left(\mathcal{F}^{-1}\left(\varphi_{j} \cdot \varphi_{0}\left(2^{-N} \cdot\right)\right)\right)(\cdot)\right|^{q}\right)^{1 / q}\right\|_{p} \\
& \geq\left\|\left(\sum_{j=0}^{N-1}\left|\left(\mathcal{F}^{-1} \varphi_{j}\right)(\cdot)\right|^{q}\right)^{1 / q}\right\|_{p} \\
& =\left\|\left(\left|\Phi_{0}\right|^{q}+\sum_{j=1}^{N-1}\left|2^{j-1} \Phi_{1}\left(2^{j-1} \cdot\right)\right|^{q}\right)^{1 / q}\right\|_{p} \\
& =\left\|\left(\left|\Phi_{0}\right|^{q}+\sum_{j=0}^{N-2}\left|2^{j} \Phi_{1}\left(2^{j} \cdot\right)\right|^{q}\right)^{1 / q}\right\|_{p} .
\end{aligned}
$$

Note that $\Phi_{1}$ has (infinitely many) vanishing moments and is thus oscillatory. Assuming w.l.o.g. $\left|\Phi_{1}\right|>\delta$ on a set $I \subset[1,2)$, with $\delta>0$ being some fixed constant, we can proceed 


$$
\begin{aligned}
\left\|f_{N}\right\|_{F_{p, q}^{0}(\mathbb{R})} & \gtrsim\left\|\left(\sum_{j=0}^{N-2}\left|2^{j} \chi_{I}\left(2^{j} \cdot\right)\right|^{q}\right)^{1 / q}\right\|_{p} \\
& =\left\|\sum_{j=0}^{N-2} 2^{j} \chi_{I}\left(2^{j} \cdot\right)\right\|_{p}=\left(\sum_{j=0}^{N-2} 2^{j p} \int_{\mathbb{R}} \chi_{I}\left(2^{j} x\right) d x\right)^{1 / p} \\
& \asymp\left(\sum_{j=0}^{N-2} 2^{j(p-1)}\right)^{1 / p} \gtrsim \begin{cases}1 & , p<1, \\
N^{1 / p} & , p \geq 1 .\end{cases}
\end{aligned}
$$

Acknowledgements We warmly thank Glenn Byrenheid, Nadi'ia Derevianko, Dinh Dũng, Dorothee Haroske, Peter Oswald, Andreas Seeger, Winfried Sickel, Vladimir N. Temlyakov, Hans Triebel, Felix Voigtlaender, and Stephane Jaffard for stimulating discussions and comments. We would also like to thank the anonymous referees for their thorough reviews which helped to noticeably improve the paper by pointing out some inaccuracies in the first version. This work has been partially supported by the ANR grant AMATIS (ANR2011 BS01 011 02) and the CNRS, Groupe de Recherche Analyse Multifractale. In addition we gratefully acknowledge support by the German Research Foundation (DFG) and the Emmy-Noether programme, Ul-403/1-1.

Funding Open Access funding enabled and organized by Projekt DEAL.

Open Access This article is licensed under a Creative Commons Attribution 4.0 International License, which permits use, sharing, adaptation, distribution and reproduction in any medium or format, as long as you give appropriate credit to the original author(s) and the source, provide a link to the Creative Commons licence, and indicate if changes were made. The images or other third party material in this article are included in the article's Creative Commons licence, unless indicated otherwise in a credit line to the material. If material is not included in the article's Creative Commons licence and your intended use is not permitted by statutory regulation or exceeds the permitted use, you will need to obtain permission directly from the copyright holder. To view a copy of this licence, visit http://creativecommons.org/licenses/by/4.0/.

\section{References}

1. Abry, P., Clausel, M., Jaffard, S., Roux, S.G., Vedel, B.: The hyperbolic wavelet transform: an efficient tool for multifractal analysis of anisotropic textures. Rev. Mat. Iberoam. 1, 1-36 (2015)

2. Abry, P., Jaffard, S., Roux, S.G., Vedel, B., Coddington, J., Daffner, L., Borgnat, P., Tremblay, N., Klein, A., Wendt, H., Messier, P.: Multiscale anisotropic texture analysis and classification of photographic prints. IEEE Signal Process. Mag. 32(4), 18-27 (2015)

3. Andrianov, A.V.: Approximation of $M H_{q}^{r}$-class functions by Haar polynomials. Math. Notes 66(3), 261-270 (1999)

4. Bonami, A., Estrade, A.: Anisotropic analysis of some Gaussian models. J. Fourier Anal. Appl. 9, 215-236 (2003)

5. Bownik, M.: Atomic and molecular decomposition of anisotropic Besov spaces. Math. Z. 250, 539-571 (2005)

6. Bownik, M., Ho, K.P.: Atomic and molecular decomposition of anisotropic Triebel-Lizorkin spaces. Trans. Am. Math. Soc. 385(4), 1469-1510 (2005)

7. Candès, E.J., Demanet, L.: The curvelet representation of wave propagators is optimally sparse. Commun. Pure Appl. Math. 58, 1472-1508 (2005)

8. Candès, E.J., Donoho, D.L.: Curvelets: a surprisingly effective nonadaptive representation for objects with edges. In: Rabut, C., Cohen, A., Schumaker, L. (eds.) Curves and Surfaces, pp. 105-120. Vanderbilt University Press, Vanderbilt (2000) 
9. Candès, E.J., Donoho, D.L.: New tight frames of curvelets and optimal representations of objects with piecewise $C^{2}$ singularities. Commun. Pure Appl. Math 56, 219-266 (2002)

10. Sharma, S., Kaur, J., Gupta, S.: Improved modified fast Haar wavelet transformation [MFHWT] based visible watermarking. In: Fourth International Conference on Computing, Communications and Networking Technologies (ICCCNT), 2013: 4-6 July 2013, Tiruchengode, India, pp. 1-5, IEEE (2013)

11. Chui, C.K., Wang, J.-Z.: On compactly supported spline wavelets and a duality principle. Trans. Am. Math. Soc. 330(2), 903-915 (1992)

12. Dũng, D., Temlyakov, V.N., Ullrich, T.: Hyperbolic Cross Approximation. arXiv e-prints (2015). arXiv:1601.03978 [math.NA]

13. Dũng, D., Ullrich, T.: N-widths and $\varepsilon$-dimensions for high-dimensional approximations. Found. Comput. Math. 13, 965-1003 (2013)

14. Demanet, L., Ying, L.: Wave atoms and sparsity of oscillatory patterns. Appl. Comput. Harmon. Anal. 23(3), 368-387 (2007)

15. Derevianko, N., Ullrich, T.: A higher order Faber spline basis for sampling discretization of functions. J. Approx. Theory. 257, 105449 (2020)

16. DeVore, R.A., Konyagin, S.V., Temlyakov, V.N.: Hyperbolic wavelet approximation. Constr. Approx. 14, 1-26 (1998)

17. Edwards, R.E.: Functional Analysis: Theory and Applications. Athena Series. Holt, Rinehart and Winston (1965)

18. Faber, G.: Über die Orthogonalfunktionen des Herrn Haar. Jahresber. Deutsch. Math. Verein. 19, 104112 (1910)

19. Fefferman, C., Stein, E.M.: Some maximal inequalities. Am. J. Math. 93, 107-115 (1971)

20. Frazier, M., Jawerth, B.: Decomposition of Besov spaces. Indiana Univ. Math. J. 34(4), 777-799 (1985)

21. Frazier, M., Jawerth, B.: A discrete transform and decompositions of distribution spaces. J. Funct. Anal. 93(1), 34-170 (1990)

22. Garrigós, G., Seeger, A., Ullrich, T.: The Haar system as a Schauder basis in spaces of Hardy-Sobolev type. J. Fourier Anal. Appl. 24(5), 1319-1339 (2018)

23. Golubov, B.I.: Best approximations of functions in the $L_{p}$ metric by Haar and Walsh polynomials. Math. USSR Sb. 16(2), 265-285 (1972)

24. Grafakos, L.: Classical Fourier Analysis. Part of the Graduate Texts in Mathematics Book Series, vol. 249, 2nd edn. Springer, New York (2008)

25. Griebel, M., Knapek, S.: Optimized general sparse grid approximation spaces for operator equations. Math. Comp. 78(268), 2223-2257 (2009). Also available as SFB611 preprint No 402

26. Guo, K., Kutyniok, G., Labate, D.: Sparse Multidimensional Representations Using Anisotropic Dilation and Shear Operators. Wavelets and Splines (Athens, GA, 2005), vol. 14, pp. 189-201. Nashboro Press, Nashville, TN (2006)

27. Haar, A.: Zur Theorie der orthogonalen Funktionensysteme. Math. Ann. 69(3), 331-371 (1910)

28. Hochmuth, R.: Wavelet characterizations for anisotropic Besov spaces. Appl. Comput. Harmon. Anal. 12, 179-208 (2002)

29. Kaiser, G.: The fast Haar transform. IEEE Potentials 17(2), 34-37 (1998)

30. Kempka, H.: Atomic, molecular and wavelet decomposition of 2-microlocal Besov and TriebelLizorkin spaces with variable integrability. Funct. Approx. Comment. Math. 43(2), 171-208 (2010)

31. Kutyniok, G., Labate, D., Lim, W.-Q., Weiss, G.: Sparse multidimensional representation using shearlets. In: Wavelets XI(San Diego, CA), volume 5914 of SPIE Proc., pp. 254-262. SPIE, Bellingham, WA (2005)

32. Kyriazis, G.: Decomposition systems for function spaces. Stud. Math. 157(2), 133-169 (2003)

33. Lakhonchai, P., Sampo, J., Sumetkijakan, S.: Shearlet transforms and directional regularities. Int. J. Wavelets Multiresolut. Inf. Process. 8(5), 743-771 (2010)

34. Liang, Y., Yang, D., Yuan, W., Sawano, Y., Ullrich, T.: A new framework for generalized Besov-type and Triebel-Lizorkin-type spaces. Dissertationes Math. (Rozprawy Mat.) 489, 1-114 (2013)

35. Nikol'skij, S.M.: Approximation of Functions of Several Variables and Imbedding Theorems, volume 205 of Grundlehren Math. Wiss. Springer Berlin, Heidelberg (1975)

36. Oswald, P.: Multivariate Haar systems in Besov function spaces. Mat. Sbornik, to appear. Available at arXiv:2002.12917 [math.FA]

37. Porwik, P., Lisowska, A.: The Haar-wavelet transform in digital image processing: its status and achievements. Mach. Graph. Vis. 13(1/2), 79-98 (2004)

38. Jernigan, M., Roeser, P.: Fast Haar Transform Algorithms. IEEE Trans. Comput. 31(2), 175-177 (1982) 
39. Romanyuk, V.S.: Constructive characteristic of Hölder classes and m-term approximations in the multiple Haar basis. Ukrainian Math. J. 66(3), 391-403 (2014)

40. Roux, S.G., Clausel, M., Vedel, B., Jaffard, S., Abry, P.: Self-similar anisotropic texture analysis: the hyperbolic wavelet transform contribution. IEEE Trans. Image Process. 22(11), 4353-4363 (2013)

41. Schertzer, D., Lovejoy, S.: Physically based rain and cloud modeling by anisotropic, multiplicative turbulent cascades. J. Geophys. Res. 92, 9693-9714 (1987)

42. Schmeisser, H.-J., Triebel, H.: Topics in Fourier Analysis and Function Spaces. A Wiley-Interscience Publication. Wiley, Chichester (1987)

43. Seeger, A., Ullrich, T.: Haar projection numbers and failure of unconditional convergence in Sobolev spaces. Math. Z. 285, 91-119 (2017)

44. Sickel, W., Triebel, H.: Hölder inequalities and sharp embeddings in function spaces of $B_{p, q}^{S}$ and $F_{p, q}^{S}$ type. Z. Anal. Anwend. 14(1), 105-140 (1995)

45. Stöckert, B.: Ungleichungen vom Plancherel-Pólya-Nikol'skij-Typ in gewichteten $L_{p}^{\Omega}$-Räumen mit gemischten Normen. Math. Nachr. 86, 19-32 (1978)

46. Temlyakov, V.N.: Approximation of Periodic Functions. Computational Mathematics and Analysis Series. Nova Science Publishers Inc, Commack, NY (1993)

47. Triebel, H.: Fourier Analysis and Function Spaces. Teubner Texte Math., Leipzig, Teubner (1977)

48. Triebel, H.: On Haar bases in Besov spaces. Serdica 4(4), 330-343 (1978)

49. Triebel, H.: Interpolation Theory, Function Spaces, Differential Operators. North-Holland, Amsterdam (1978)

50. Triebel, H.: Wavelet bases in anisotropic function spaces. Funct. Spaces Differ. Oper. Nonlinear Anal. 18, 529-550 (2004)

51. Triebel, H.: Theory of Function Spaces III, volume 100 of Monogr. Math. Birkhäuser Verlag, Basel (2006)

52. Triebel, H.: Theory of Function Spaces. Modern Birkhäuser Classics, Springer Basel (2010)

53. Triebel, H.: Bases in function Spaces, Sampling, Discrepancy, Numerical Integration. Vol. 11, European Mathematical Society (2010)

54. Triebel, H.: Theory of Function Spaces IV, volume 107 of Springer Monogr. Math. Birkhäuser, Springer Nature Switzerland, Cham (2020)

55. Ullrich, M., Ullrich, T.: The role of Frolov's cubature formula for functions with bounded mixed derivative. SIAM J. Numer. Anal. 54, 969-993 (2016)

56. Ullrich, T.: Continuous characterizations of Besov-Lizorkin-Triebel spaces and new interpretations as coorbits. J. Funct. Spaces Appl. (2012)

57. Ullrich, T., Rauhut, H.: Generalized coorbit space theory and inhomogeneous function spaces of Besov-Lizorkin-Triebel type. J. Funct. Anal. 260(11), 3299-3362 (2011)

58. Voigtlaender, F.: Embeddings of decomposition spaces. Mem. Am. Math. Soc., to appear. Available at arXiv:1605.09705 [math.FA]

59. Vybíral, J.: Function spaces with dominating mixed smoothness. Dissertationes Math. (Rozprawy Mat.) 436, 1-73 (2006)

Publisher's Note Springer Nature remains neutral with regard to jurisdictional claims in published maps and institutional affiliations. 